Celso de Carvalho Noronha Neto

\title{
INTEGRAÇÃO DAS EQUAÇÕES DIFERENCIAIS DO FILTRO DIGITAL DE BUTTERWORTH MEDIANTE ALGORITMO DE QUADRATURA NUMÉRICA DE ORDEM ELEVADA
}

Dissertação apresentada à Escola de Engenharia de São Carlos da Universidade de São Paulo, como parte dos requisitos para a obtenção do título de Mestre em Engenharia de Estruturas.

Orientador: Prof. Tit. Dr. José Elias Laier 
Aos meus pais e à Joseana 


\section{Sumário}

Lista de símbolos i

Resumo iii

Abstract iv

Capítulo I - Introdução 1

Capítulo II - Sistema de um grau de liberdade 5

2.1. Introdução 5

2.2. Equação de movimento 6

2.3. Solução homogênea 7

2.4. Solução particular para carregamento senoidal 8

2.5. Solução particular para carregamento cossenoidal 10

2.6. Análise da solução geral 11

Capítulo III - Transformada de Fourier 14

3.1. Introdução 14

3.2. Série de Fourier 15

3.3. Transformada de Fourier 15

3.4. Propriedades da transformada de interesse na resolução de equações diferenciais 17

3.5. Transformada da função senoidal 18

3.6. Aplicação na equação de movimento 19

3.7. Aplicação da transformada em uma equação diferencial de ordem genérica 20

Capítulo IV - Equação de filtragem 22

4.1. Introdução 22

4.2. Conceituação de filtro 23

4.3. Tipos de filtro 
4.4. Tipos de equação para $H(\omega)$

4.5. Função $\mathrm{H}(\omega) \_26$

4.6. Aplicação para uma entrada senoidal___ 26

4.7. Filtro de Butterworth e modelação de $|H(\omega)| \_29$

4.8. Escolha dos parâmetros__ 30

4.9. Estudo dos pólos___ 33

4.10. Filtros e equações diferenciais correspondentes___ 35

Capítulo V - Filtro digital com quadratura numérica de segunda ordem (trapezoidal)__ 40

5.1. Introdução__ 40

5.2. Analogia com a equação de movimento___ 41

5.3. Método Newmark de passo duplo___ 42

5.4. Método Newmark de passo simples___ 44

5.5. Exemplos de aplicação___ 46

5.6. Formulação matricial reduzida da equação diferencial____ 48

5.7. Aplicação para filtro de dois pólos___ 50

5.8. Filtragem utilizando número de pólos mais elevado___ 52

Capítulo VI - Filtro digital com quadratura numérica de ordem superior (operador hermitiano)__ 55

6.1. Introdução___ 55

6.2. Operador hermitiano com derivada de segunda ordem __ 56

6.3. Caso geral de operadores___ 58

6.4. Aplicação no algoritmo de dois pólos___ 59

6.5. Discretização da derivada do sinal de entrada___ 60

6.6. Aplicação para filtro de dois pólos___ 62

6.7. Algoritmo hermitiano para filtro de Butterworth de ordem genérica_64

6.8. Verificação da eficiência do filtro utilizando número de pólos mais elevados 65 
Capítulo VII - Análise comparativa dos algoritmos 68

7.1. Introdução 68

7.2. $1^{\circ}$ exemplo : Comparação de entrada composta por uma única senóide 69

7.3. $2^{\circ}$ exemplo: Comparação do comportamento dos algoritmos para diferentes valores de $\Delta t$ 70

7.4. $3^{\circ}$ exemplo: Análise da entrada amortecida 72

7.5. $4^{\circ}$ exemplo: Análise da entrada contendo parcela randômica 73

7.6. $5^{\circ}$ exemplo: Análise da entrada randômica pura 76

Capítulo VIII - Algoritmo de filtragem proveniente da transformada Z 78

8.1. Introdução 78

8.2. Transformada de Laplace 79

8.3. Transformada $Z$ 79

8.4. Transformação bilinear 81

8.5. Polinômio de Butterworth 82

8.6. Desenvolvimento de filtro digital de dois pólos 83

8.7. Comparação dos resultados 84

Capítulo IX - Observações finais e conclusões 86

Referências bibliográficas 88 


\section{Lista de símbolos}

$\mathrm{k}=$ constante de rigidez elástica

$\mathrm{c}=$ amortecimento

$\mathrm{m}=$ massa

$\mathrm{t}=$ tempo

$F_{k}=$ força de mola

$F_{c}=$ força de amortecimento

$\rho(t)=$ movimento adimensional de resposta

$\mathrm{x}_{\mathrm{e}}=$ deslocamento estático de referência

$\omega=$ freqüência angular

$\omega_{n}=$ freqüência angular natural

$\gamma=$ coeficiente de amortecimento

$\rho_{\mathrm{h}}(\mathrm{t})=$ solução homogênea adimensional do sistema

$\mathrm{C}_{1}, \mathrm{C}_{2}=$ constantes de integração

$\omega_{1}=$ freqüência natural amortecida

$\rho_{p}(t)=$ solução particular adimensional do sistema

C = fator de amplificação

$\varphi=$ ângulo de defasagem

$\alpha=$ relação entre freqüência de entrada e freqüência de resposta

$a_{i}, b_{i}=$ coeficientes

$A(\omega), B(\omega)=$ integrais de Fourier

$F(\omega)$ transformada de Fourier da função $f(t)$

$\delta(\omega)=$ delta de Dirac

$x(t)=$ sinal de entrada

$\mathrm{y}(\mathrm{t})=$ sinal de saída

$X(\omega)=$ transformada de Fourier do sinal de entrada

$Y(\omega)=$ transformada de Fourier do sinal de saída

$H(\omega)=$ função de resposta em freqüência

$\omega_{c}=$ freqüência de corte 
$A=$ amplitude do sinal de entrada

$\mathrm{N}$ = número de pólos do filtro

$H_{N}(\omega)$ = função de resposta em freqüência para um filtro de $N$ pólos

$H_{\text {par }}(\omega)$ = função de resposta em freqüência para um filtro com um

número par de pólos

$H_{\text {impar }}(\omega)=$ função de resposta em freqüência para um filtro com um número ímpar de pólos

$P(\omega)=$ transformada de Fourier da equação diferencial de filtragem

$P_{N}(\omega)=$ transformada de Fourier da equação diferencial de filtragem com $\mathrm{N}$ pólos

$\mathrm{x}_{\mathrm{k}}=\mathrm{k}$-gésimo valor discretizado do sinal de entrada

$y_{k}=k$-gésimo valor discretizado do sinal de saída

$\Delta \mathrm{t}=$ diferença de tempo entre uma leitura e outra feita pelo aparelho de mensuração

$\theta=$ ângulo cujo valor é $\omega_{c} \Delta t$

$\{X\}_{\mathrm{i}}=$ vetor de entrada do sistema matricial

$\{Y\}_{i}=$ vetor de resposta do sistema matricial

$[\mathrm{A}]=$ matriz de redução

$[l]=$ matriz identidade

$\mathrm{O}\left(\Delta \mathrm{t}^{\mathrm{j}}\right)=$ ordem de erro de grau $\mathrm{j}$

$\mathrm{T}=$ período de oscilação

$\varepsilon(j)=$ parcela randômica que varia de $-\mathrm{j} a+j$

$F(s)=$ transformada de Laplace da função $f(t)$

$F[z]=$ transformada $Z$ da função $f[n]$

$x[n], y[n]=$ discretização das funções $x(t)$ e $y(t)$ respectivamente

$Y[z]=$ transformada $Z$ da função de entrada

$X[z]=$ transformada $Z$ da função de saída 


\section{Resumo}

NORONHA Neto, Celso C. (2003). Integração das equações diferenciais do filtro digital de Butterworth mediante algoritmo de quadratura numérica de ordem elevada. São Carlos. 89p. Dissertação (Mestrado) - Escola de Engenharia de São Carlos, Universidade de São Paulo.

Neste trabalho se apresenta o desenvolvimento de algoritmos hermitianos de integração das equações diferenciais do filtro digital de Butterworth mediante operadores de integração numérica de ordem elevada com passo único.

A teoria do filtro de Butterworth é apresentada mediante o emprego da transformada de Fourier. Exemplos de aplicação apresentados através destes algoritmos mostram que os resultados são, como esperado, mais precisos que os resultantes dos métodos usuais presentes na literatura especializada.

Palavras-chave: processamento de sinais, filtro digital, Butterworth, hermitiano, solução numérica de equações diferenciais. 
Abstract

NORONHA Neto, Celso C. (2003). Integration of the Butterworth digital filter's differential equations using numerical algorithm of high order integrator. São Carlos. 89p. Dissertação (Mestrado) - Escola de Engenharia de São Carlos, Universidade de São Paulo.

In this work is presented the development of hermitian algorithm for integration of the Butterworth digital filter's differential equations by means of high order numerical one step operators.

The Butterworth filter's theory is presented based on the Fourier transform. Numerical examples show that the results of the developed hermitian algorithm are more accurate than the usual methods present in the specialized literature, as expected.

Keywords: signal processing, digital filter, Butterworth, hermitian, numerical solution of differential equations. 


\section{Capítulo I}

\section{INTRODUÇÃO}

A análise dinâmica das estruturas, como sabido, envolve o estudo do movimento e do comportamento de um ou mais elementos da estrutura sob efeito de uma solicitação dinâmica, seja esta uma solicitação por impacto, com descrição eventualmente de grande complexidade, ou mesmo um carregamento bem mais simples, como o caso de carregamento harmônico. Um elemento estrutural ao oscilar pode apresentar, em termos previstos pela teoria, uma infinidade de modos de vibração, conforme ilustra a figura 1 , sendo que a influência do primeiro modo, como sabido, é bem maior que a do segundo modo; o qual, por sua vez, é bem maior que a influência do terceiro modo, e assim por diante.

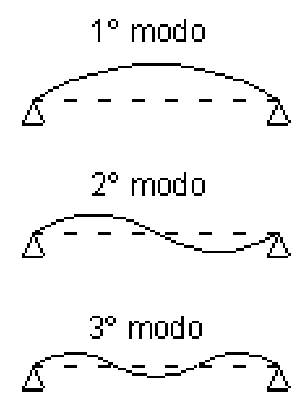

Fig. 1- Modos de vibração de uma barra

Em geral, para efeitos da prática, na grande maioria dos casos de interesse, a análise dinâmica pode ser realizada levando-se em conta apenas o primeiro modo de vibração, que recebe o nome de modo principal ou modo fundamental. Os demais modos de vibração, em geral, apresentam contribuição de menor significado, podendo ser até negligenciados nos estudos. Por essa razão, é conveniente promover uma eliminação de sinais correspondentes contidos no 
movimento da estrutura, mediante uma filtragem apropriada. Essa tarefa é essencialmente uma das desempenhadas pelos chamados filtros de freqüência, em particular o filtro de Butterworth, a merecer especial atenção.

Basicamente, conhecendo-se a freqüência fundamental do elemento estrutural, pode-se apontar a solicitação a qual este está sujeito, conhecendo-se suas características físicas e geométricas, ou então verificar suas características físicas conhecendo-se a solicitação e as características geométricas. Em outras palavras, a estrutura nos revela muita coisa a partir de uma solicitação dinâmica, basta que sejam colhidas apenas informações de interesse.

Por outro lado, uma outra aplicação notável da filtragem de freqüências é dada nos estudo da acústica, tendo-se em vista que a percepção da intensidade sonora é essencialmente um fenômeno dependente da freqüência, uma vez que a sensibilidade do ouvido humano varia ao longo do espectro audível, exigindo-se uma média com ponderação para resultar numa medida que melhor reflita a audibilidade percebida na realidade. Assim sendo, nota-se que a filtragem de freqüências está presente em muitos temas do cotidiano, sendo tema de grande interesse em vários ramos da engenharia.

Os filtros digitais, versão numérica dos filtros físicos, foram primeiramente um assunto de interesse maior na área da engenharia elétrica, sendo que os correspondentes operadores têm sido muito pouco divulgados no meio, por exemplo, da engenharia de estruturas. Mesmo porque, as aplicações na engenharia elétrica estão mais voltadas para a filtragem de várias freqüências em uma faixa de freqüência limitada, denominada banda; e nos estudos da dinâmica das estruturas os interesses estão mais voltados para a descrição do comportamento no decorrer do tempo de massas em movimento devido à ação de uma carga aplicada. Então, o objetivo deste trabalho é compreender em maior detalhe os procedimentos de cálculo envolvidos na filtragem digital de freqüências, bem como especialmente o desenvolvimento dos operadores correspondentes.

Todavia, vale ressaltar que, dentro da área de conhecimento do processamento de sinais, são de grande interesse para a dinâmica das estruturas os procedimentos de análise de sinal, tais como os da transformada discreta de Fourier, conhecida pela sigla DFT (Discrete Fourier Transform), que proporciona uma versão discretizada da transformada de Fourier; o mais recente algoritmo de Wavelet, que consiste numa convolução apropriada na função que descreve um 
sinal; a chamada transformada Z, que promove uma discretização da transformada de Laplace; e tantos outros. Além disso, mais importante a observar é que, ao se desenvolver os algoritmos de filtragem digital de Butterworth, tem-se também em mente complementá-los.

Cumpre também assinalar que não é objeto deste trabalho a elaboração de estudos sobre a parte física dos filtros elétricos de freqüência, presentes nos aparelhos de mensuração de grandezas da dinâmica das estruturas (acelerômetros, sismômetros e decibelímetros), inclusive os chamados antialiasing, e sim apenas enfocar os cálculos realizados na engenharia elétrica para a filtragem digital de freqüências.

Para melhor visualização do fenômeno da filtragem em freqüência, ilustra-se na figura 2 um sinal de entrada com componentes de várias freqüências, e o resultado após o processo de filtragem, quando então as componentes de maior freqüência já estão eliminadas do sinal.
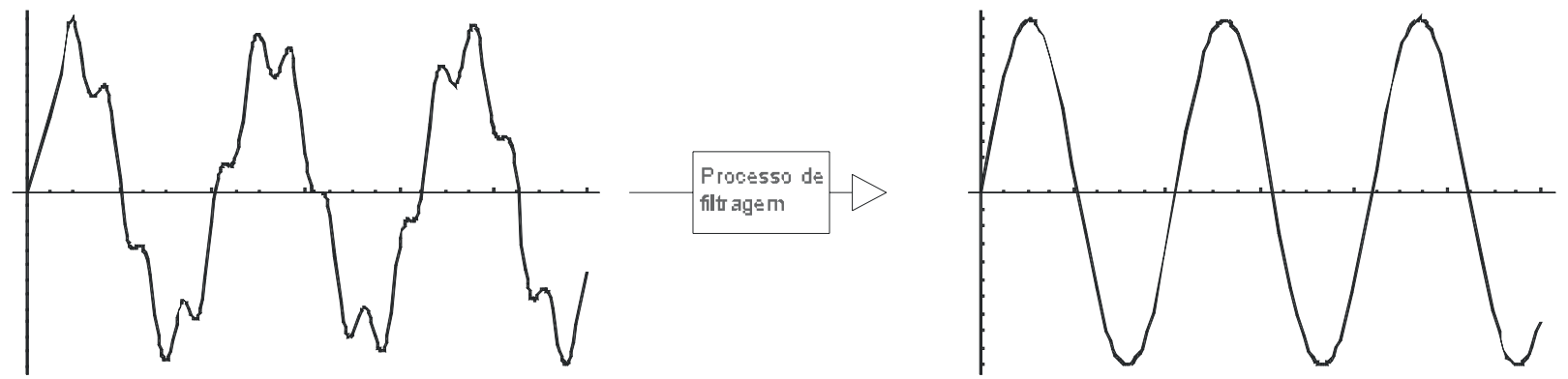

Fig. 2 - Exemplo de filtragem

Assim, o processo de filtragem inicia-se pela definição da freqüência angular de corte $\omega_{c}$ desejada. No caso de um sinal típico da dinâmica das estruturas, a freqüência de corte deve ser arbitrada de sorte a permitir a passagem de apenas o modo fundamental, por exemplo. Para tanto, o estudo inicia-se pela formulação do problema no domínio da freqüência, o que se faz por meio do emprego da transformada de Fourier. Por meio da teoria da transformada de Fourier surge então uma relação algébrica entre a transformada do sinal de entrada e a transformada do sinal de saída, sendo que esta relação algébrica no domínio da freqüência tem uma correspondente equação diferencial no domínio do tempo, cuja integração resulta no sinal filtrado.

Para ilustrar os procedimentos numéricos de resolução de uma genérica equação diferencial correspondente, escolhe-se de início a equação de movimento, 
pois é esta a equação mais trabalhada na dinâmica das estruturas. Assim, no capítulo II é analisado um sistema de um grau de liberdade considerando-se carregamentos harmônicos senoidais e cossenoidais. Com isso, busca-se uma sugestiva analogia entre uma equação de filtragem digital e a correspondente equação de movimento, facilitando-se pois o entendimento mais direto do processo de filtragem. Conseqüentemente, cria-se neste capítulo um filtro mecânico, porém de difícil modelagem prática devido ao fato de se ter dificuldades para calibração do amortecimento. No capítulo III a transformada de Fourier é desenvolvida no sentido de aclarar a operação de maior interesse no domínio da freqüência, bem como exibir com maiores detalhes as relações entre as operações de filtragem em freqüência e as correspondentes equações diferenciais no domínio do tempo.

A generalização do processo de filtragem para uma equação diferencial de ordem qualquer é então elaborada no capítulo IV, sendo que a atenção é mais voltada agora para a técnica de escolha dos coeficientes da equação diferencial que proporciona melhor desempenho para filtragem. No capítulo $V$ desenvolve-se, de maneira um tanto apropriada, o clássico algoritmo denominado algoritmo trapezoidal, que é o algoritmo numérico preconizado na literatura de engenharia elétrica. No capítulo VI encontra-se o desenvolvimento de um algoritmo provido de quadratura numérica de ordem elevada, que é o algoritmo proposto no presente trabalho; e cuja dedução, um tanto mais avantajada no montante de operações, apresenta um grau de convergência de maior ordem. Trata-se de um algoritmo da família dos chamados algoritmos hermitianos, por envolver combinações de valores da função e de suas derivadas. Estes algoritmos utilizam, pois, como incógnitas numéricas não só os valores da função mas também suas derivadas, diferentemente dos algoritmos dos filtros digitais baseados na transformada Z, que utilizam apenas valores da função sinal incógnita a processar numericamente.

No capítulo VII encontram-se análises comparativas entre os algoritmos elaborados no tocante aos seus erros locais, com diversos tipos de sinais, inclusive sinais com parcela randômica.

A título de anexo, o capítulo VIII apresenta o embasamento teórico para a formulação de filtros digitais de freqüência de acordo com a literatura especializada. Trata-se da transformada $Z$, proveniente da transformada de Laplace. Finalizando, o capítulo IX é dedicado à considerações finais, concluindo a dissertação. 


\section{Capítulo II}

\section{SISTEMA DE UM GRAU DE LIBERDADE}

\subsection{INTRODUÇÃO}

O estudo detalhado da equação de movimento de um sistema de um grau de liberdade, como ilustrado na figura 2.1, constitui-se em ponto de partida interessante para o estudo do processo de filtragem em freqüência do tipo clássico de Butterworth[1], tendo-se em vista que há uma correspondência notável com o filtro de dois pólos, mediante ajuste adequado de parâmetros.

A figura 2.1 exibe um sistema de um grau de liberdade, onde, conforme a notação clássica, k é a rigidez do sistema, c o amortecimento, $\mathrm{m}$ a inércia, $x(t)$ a resposta e P.f(t) a solicitação, sendo $P$ uma carga de referência $e$ $\mathrm{f}(\mathrm{t})$ a função que descreve a variação da solicitação no tempo[2].

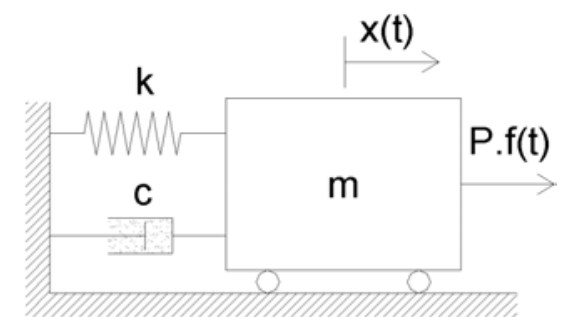

Fig. 2.1 - Sistema de um grau de liberdade

É oportuno assinalar que a solicitação P.f(t) pode ser entendida como um sinal de entrada e a resposta $x(t)$ o sinal de saída (depois de passado pelo filtro).

Apresenta-se em seguida a equação de equilíbrio dinâmico do sistema, bem como sua redação na forma adimensional clássica. A solução homogênea e também as particulares no caso senoidal e cossenoidal são 
apresentadas na seqüência, finalizando com as soluções completas desses casos harmônicos.

\subsection{EQUAÇÃO DE MOVIMENTO}

A figura 2.2 exibe a massa genérica do sistema e as ações nela atuantes, quais sejam, a força de mola $F_{k}(t)$, a força de amortecimento $F_{c}(t)$ e a força atuante P.f(t).

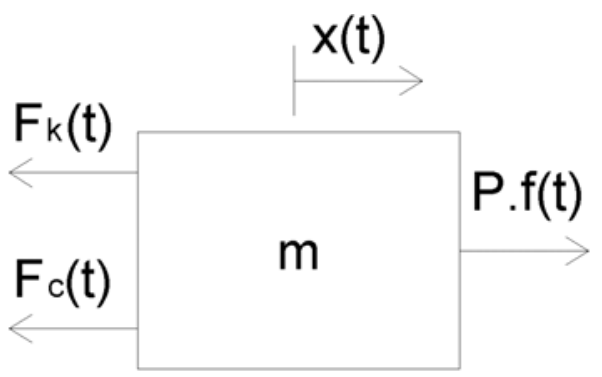

Fig. 2.2 - Forças atuantes

Tendo-se em vista a segunda lei de Newton, o equilíbrio dinâmico do sistema escreve-se:

$$
F(t)-F_{c}(t)-F_{k}(t)=m . \ddot{x}(t)
$$

onde se emprega a clássica notação por pontos superiores para indicar a derivação na variável tempo; ou ainda:

$$
\mathrm{m} . \ddot{\mathrm{x}}(\mathrm{t})+\mathrm{c} \cdot \dot{\mathrm{x}}(\mathrm{t})+\mathrm{k} \cdot \mathrm{x}(\mathrm{t})=\text { P.f }(\mathrm{t})
$$

sendo assumido, pois, a modelagem dita linear (massa, amortecimento e rigidez constantes, e relação linear para força de mola e de amortecimento).

A equação (2.2) ganha, na forma adimensional, a seguinte escrita:

$$
\ddot{\rho}(\mathrm{t})+2 \gamma \omega_{\mathrm{n}} \dot{\rho}(\mathrm{t})+\omega_{\mathrm{n}}^{2} \rho(\mathrm{t})=\omega_{\mathrm{n}}^{2} \mathrm{f}(\mathrm{t})
$$

onde:

$$
\begin{aligned}
& x_{e}(t)=\frac{P}{k} \\
& \rho(t)=\frac{x(t)}{x_{e}(t)} \\
& \omega_{n}=\sqrt{\frac{k}{m}} \\
& \gamma=\frac{c}{2 m \omega_{n}}
\end{aligned}
$$


sendo $x_{e} \circ$ deslocamento estático de referência, $\rho(t) \quad o$ movimento adimensional, $\omega_{n}$ a freqüência angular natural e $\gamma$ o coeficiente de amortecimento.

Como se sabe, a solução geral da equação (2.3) pode ser separada em duas partes, sendo que a primeira corresponde ao caso do movimento sem carregamento aplicado e atendendo às condições iniciais do movimento (posição e velocidade). A segunda parte da solução geral, chamada de solução particular, depende apenas do carregamento aplicado.

\subsection{SOLUÇÃO HOMOGÊNEA}

A equação de movimento na ausência de carregamento, ou seja:

$$
\ddot{\rho}_{h}(t)+2 \gamma \omega_{n} \dot{\rho}_{h}(t)+\omega_{n}^{2} \rho_{h}(t)=0
$$

tem, como sabido, solução tipo exponencial:

$$
\rho_{\mathrm{h}}(\mathrm{t})=A \mathrm{e}^{\lambda \mathrm{t}}
$$

Substituindo o expresso em (2.6) na equação de movimento (2.5), obtém-se duas soluções para o parâmetro $\lambda$, quais sejam:

$$
\begin{aligned}
& \lambda_{1}=-\gamma \omega_{\mathrm{n}}+\omega_{\mathrm{n}} \sqrt{\gamma^{2}-1} \\
& \lambda_{2}=-\gamma \omega_{\mathrm{n}}-\omega_{\mathrm{n}} \sqrt{\gamma^{2}-1}
\end{aligned}
$$

Assim sendo, a solução homogênea pode então ser expressa por:

$$
\rho_{h}(t)=\left\lfloor A_{1} e^{\sqrt{\gamma^{2}-1} \omega_{n} t}+A_{2} e^{-\sqrt{\gamma^{2}-1} \omega_{n} t}\right\rfloor e^{-\gamma \omega_{n} t}
$$

onde $A_{1}$ e $A_{2}$ são as constantes de integração.

Examinando-se o expresso em (2.8), verifica-se que, no caso onde o coeficiente de amortecimento $\gamma$ é menor que a unidade, que é o de interesse, as potências tornam-se complexas, e, segundo as fórmulas de Euler[2], a solução (2.8) adquire uma nova redação: 


$$
\rho_{h}(t)=\left[C_{1} \cos \left(\omega_{1} t\right)+C_{2} \operatorname{sen}\left(\omega_{1} t\right)\right] e^{-\gamma \omega_{n} t}
$$

onde:

$$
\begin{aligned}
& C_{1}=A_{1}+A_{2} \\
& C_{2}=i\left(A_{1}-A_{2}\right) \\
& \omega_{1}=\sqrt{1-\gamma^{2}} \omega_{n}
\end{aligned}
$$

na nomenclatura da dinâmica estrutural, $\omega_{1}$ é denominada freqüência natural amortecida.

\subsection{SOLUÇÃO PARTICULAR PARA CARREGAMENTO SENOIDAL}

Para simplificar a exposição, admite-se que a solução particular da equação (2.3) no caso de variação senoidal de freqüência angular $\omega$, ou seja $f(t)=\operatorname{sen}(\omega t)$, pode ser expressa na forma:

$$
\rho_{p}(t)=C \cdot \operatorname{sen}(\omega t-\varphi)
$$

onde C é o fator de amplificação e $\varphi$ o ângulo de defasagem.

Substituindo-se (2.11) em (2.3) tem-se:

$$
\left(-C \omega^{2} \operatorname{sen}(\omega t-\varphi)\right)+2 \gamma \omega_{n}(C \omega \cos (\omega t-\varphi))+\omega_{n}^{2}(C \cdot \operatorname{sen}(\omega t-\varphi))=\omega_{n}^{2} \operatorname{sen}(\omega t)
$$

ou ainda:

$$
C\left[\left(\omega_{n}^{2}-\omega^{2}\right) \operatorname{sen}(\omega t-\varphi)+2 \gamma \omega_{n} \omega \cos (\omega t-\varphi)\right]=\omega_{n}^{2} \operatorname{sen}(\omega t)
$$

cuja solução implica em:

$$
C\left\{\begin{array}{l}
\operatorname{sen}(\omega t)\left[\cos (\varphi)\left(\omega_{n}^{2}-\omega^{2}\right)+\operatorname{sen}(\varphi) 2 \gamma \omega_{n} \omega\right]+\cdots \\
+\cos (\omega t)\left[\cos (\varphi) 2 \gamma \omega_{n} \omega-\operatorname{sen}(\varphi)\left(\omega_{n}^{2}-\omega^{2}\right)\right]
\end{array}\right\}=\omega_{n}^{2} \operatorname{sen}(\omega t)
$$

resultando:

$$
\begin{aligned}
& C\left[\cos (\varphi)\left(\omega_{n}^{2}-\omega^{2}\right)+\operatorname{sen}(\varphi) 2 \gamma \omega_{n} \omega\right]=\omega_{n}^{2} \\
& C\left[\cos (\varphi) 2 \gamma \omega_{n} \omega-\operatorname{sen}(\varphi)\left(\omega_{n}^{2}-\omega^{2}\right)\right]=0
\end{aligned}
$$


A solução não trivial $(C \neq 0)$ da primeira de (2.15) conduz a:

$$
\cos (\varphi) 2 \gamma \omega_{n} \omega-\operatorname{sen}(\varphi)\left(\omega_{n}^{2}-\omega^{2}\right)=0
$$

o que implica em:

$$
\varphi=\arctan \left(\frac{2 \gamma \omega_{\mathrm{n}} \omega}{\omega_{\mathrm{n}}^{2}-\omega^{2}}\right)
$$

ou ainda:

$$
\begin{aligned}
& \operatorname{sen}(\varphi)=\frac{2 \gamma \omega_{n} \omega}{\sqrt{\left(\omega_{n}^{2}-\omega^{2}\right)^{2}+\left(2 \gamma \omega_{n} \omega\right)^{2}}} \\
& \cos (\varphi)=\frac{\omega_{n}^{2}-\omega^{2}}{\sqrt{\left(\omega_{n}^{2}-\omega^{2}\right)^{2}+\left(2 \gamma \omega_{n} \omega\right)^{2}}}
\end{aligned}
$$

Finalmente, a substituição de (2.18) na primeira de (2.15) fornece o fator de amplificação:

$$
C=\frac{1}{\sqrt{\left(1-\alpha^{2}\right)^{2}+(2 \gamma \alpha)^{2}}}
$$

onde:

$$
\alpha=\frac{\omega}{\omega_{\mathrm{n}}}
$$

A solução particular, nesse caso, passa a ser expressa por:

$$
\rho_{\mathrm{p}}(\mathrm{t})=\frac{\operatorname{sen}(\omega \mathrm{t}-\varphi)}{\sqrt{\left(1-\alpha^{2}\right)^{2}+(2 \gamma \alpha)^{2}}}
$$

onde:

$$
\varphi=\arctan \left(\frac{2 \gamma \alpha}{1-\alpha^{2}}\right)
$$

estabelecendo-se então a solução particular de uma entrada senóide. 


\subsection{SOLUÇÃO PARTICULAR PARA CARREGAMENTO COSSENOIDAL}

Analogamente ao procedimento anterior, a realização da solução de uma solicitação agora cossenoidal é feita reescrevendo-se (2.15) da seguinte forma:

$$
\begin{aligned}
& C\left[\cos (\varphi)\left(\omega_{n}^{2}-\omega^{2}\right)+\operatorname{sen}(\varphi) 2 \gamma \omega_{n} \omega\right]=0 \\
& C\left[\cos (\varphi) 2 \gamma \omega_{n} \omega-\operatorname{sen}(\varphi)\left(\omega_{n}^{2}-\omega^{2}\right)\right]=\omega_{n}^{2}
\end{aligned}
$$

resultando, pois:

$$
\cos (\varphi)\left(\omega_{n}^{2}-\omega^{2}\right)+\operatorname{sen}(\varphi) 2 \gamma \omega_{n} \omega=0
$$

com:

$$
\varphi=\arctan \left(-\frac{1-\alpha^{2}}{2 \gamma \alpha}\right)
$$

sendo oportuno notar que o ângulo $\varphi$ dado por (2.25) é defasado de $\pm \pi / 2 \mathrm{em}$ relação ao ângulo dado por (2.22). Assim sendo, a solução pode ser expressa por:

$$
\rho_{p}(t)=\frac{\operatorname{sen}(\omega t-\varphi)}{\sqrt{\left(1-\alpha^{2}\right)^{2}+(2 \gamma \alpha)^{2}}}
$$

com:

$$
\varphi=\arctan \left(-\frac{1-\alpha^{2}}{2 \gamma \alpha}\right)
$$

ou, como mais apresentado na literatura:

$$
\rho_{p}(t)=\frac{\cos (\omega t-\varphi)}{\sqrt{\left(1-\alpha^{2}\right)^{2}+(2 \gamma \alpha)^{2}}}
$$

com

$$
\varphi=\arctan \left(\frac{2 \gamma \alpha}{1-\alpha^{2}}\right)
$$

encerrando-se a formulação nos aspectos de interesse. 


\subsection{ANÁLISE DA SOLUÇÃO GERAL}

A combinação da solução homogênea com a solução particular resulta na solução geral da equação de movimento. No caso de carregamento senoidal, por exemplo, tem-se:

$$
\rho(t)=\left[C_{1} \cos \left(\omega_{1} t\right)+C_{2} \operatorname{sen}\left(\omega_{1} t\right)\right] e^{-\gamma \omega_{n} t}+\frac{\operatorname{sen}(\omega t-\varphi)}{\sqrt{\left(1-\alpha^{2}\right)^{2}+(2 \gamma \alpha)^{2}}}
$$

sendo que a solução homogênea é responsável pela parte transitória do movimento, porquanto afetada por um exponencial negativo crescente com o tempo, e a solução particular a parte permanente da solução, visto que não é afetada por termos com exponencial negativo no domínio do tempo.

No processo de filtragem, as condições iniciais do sinal são consideradas nulas, como no caso de vibração forçada, de sorte a não se ter em conta o efeito de eventuais carregamentos externos. A nulidade das condições iniciais implica em:

$$
\begin{aligned}
& {\left[C_{1} \cos (0)+C_{2} \operatorname{sen}(0)\right] e^{0}+\frac{\operatorname{sen}(-\varphi)}{\sqrt{\left(1-\alpha^{2}\right)^{2}+(2 \gamma \alpha)^{2}}}=0} \\
& {\left[-C_{1} \operatorname{sen}(0)+C_{2} \cos (0)\right] \omega_{1} e^{0}-\gamma \omega_{n}\left[C_{1} \cos (0)+C_{2} \operatorname{sen}(0)\right] e^{0}+\frac{\cos (-\varphi)}{\sqrt{\left(1-\alpha^{2}\right)^{2}+(2 \gamma \alpha)^{2}}}=0}
\end{aligned}
$$

cuja solução em $\mathrm{C}_{1}$ e $\mathrm{C}_{2}$, levada em (2.30), permite redigir a solução geral:

$$
\rho(t)=\frac{\omega_{1} \operatorname{sen}(\omega t-\varphi)+\left[\operatorname{sen}\left(\omega_{1} t\right)\left(\gamma \omega_{n} \operatorname{sen}(\varphi)-\omega \cos (\varphi)\right)+\omega_{1} \cos \left(\omega_{1} t\right) \operatorname{sen}(\varphi)\right] \mathrm{e}^{-\gamma \omega_{n} t}}{\omega_{1} \sqrt{\left(\alpha^{2}-1\right)^{2}+(2 \gamma \alpha)^{2}}}
$$

indicando-se que a parte permanente da solução é dada por:

$$
\rho(t)=\frac{\operatorname{sen}(\omega t-\varphi)}{\sqrt{\left(\alpha^{2}-1\right)^{2}+(2 \gamma \alpha)^{2}}}
$$


Nos problemas correntes da dinâmica estrutural, os valores do amortecimento $\gamma$ são baixos, algo como 0,01 a 0,02 grosso modo. Todavia, no processo de filtragem de Butterworth o valor de $\gamma$ é arbitrado como $\sqrt{2} / 2$, ganhando a equação (2.33) a escrita:

$$
\rho(t)=\frac{1}{\sqrt{1+\alpha^{4}}} \operatorname{sen}(\omega t-\varphi)
$$

A figura 2.3 ilustra a variação da amplitude da resposta adimensional segundo equação (2.34), mostrando-se que, para $\alpha$ menor que a unidade, ou seja $\omega<\omega_{n}$, a resposta é próxima da unidade, e, para $\omega>\omega_{n}(\alpha>1)$ a resposta fica bastante reduzida, podendo ser entendida a freqüência $\omega=\omega_{n}$ como sendo a freqüência de corte do filtro, dito passa-baixa nesse caso.

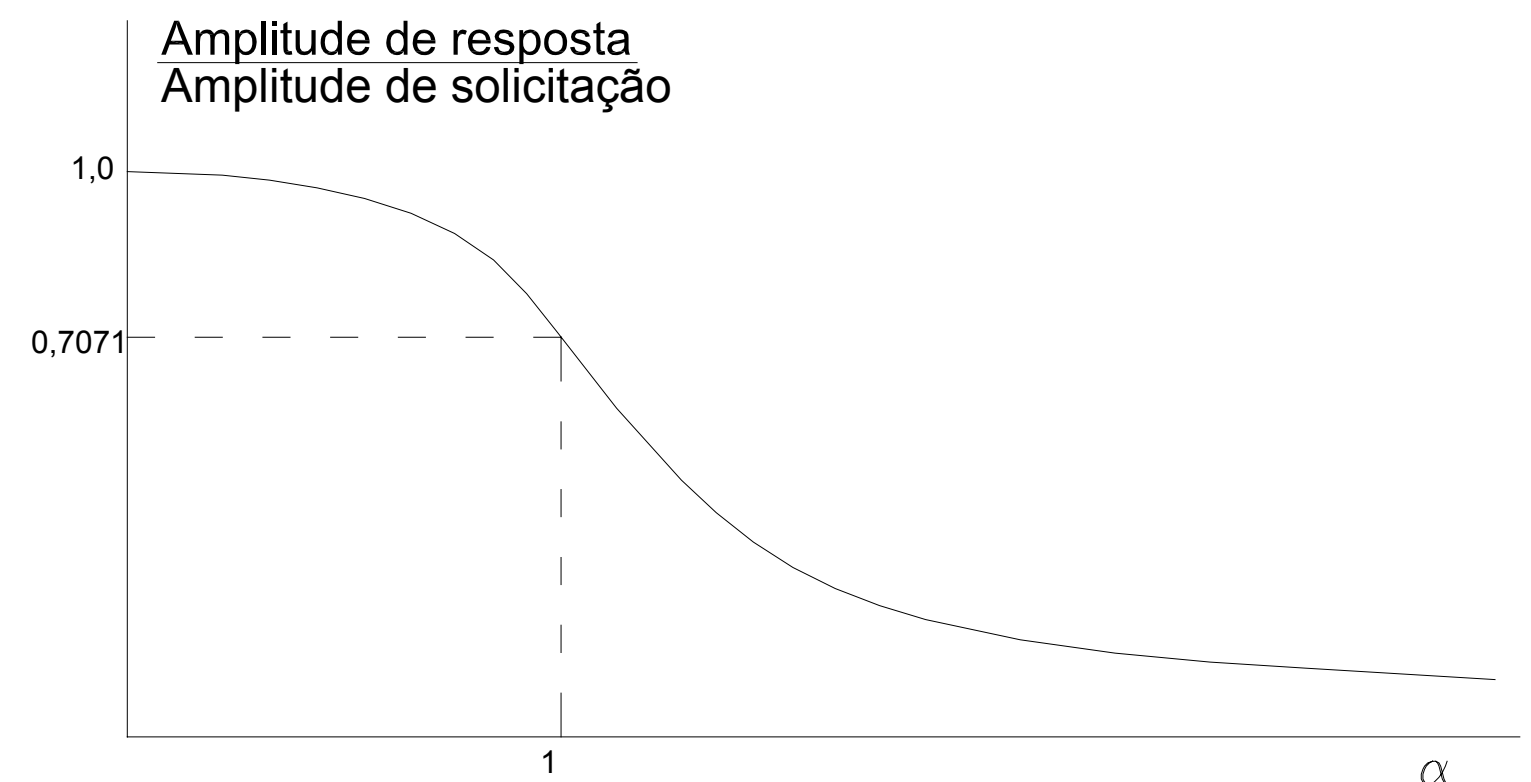

Fig. 2.3 - Fator de ampliação da amplitude

Na visão de um engenheiro de estruturas, a figura 2.3 mostra que, para uma freqüência de carregamento menor que a natural, a resposta tem seu movimento com amplitude muito próxima da amplitude da solicitação, enquanto que, se o carregamento tiver freqüência maior que a natural do sistema, a amplitude é reduzida drasticamente. Já na visão de um engenheiro elétrico, o gráfico lembra um filtro que retém sinais de freqüência elevada, deixando passar apenas o de baixa freqüência, ou seja, trata-se de um filtro do tipo passa-baixa. 
Vale registrar que o valor particular do coeficiente de amortecimento dado por:

$$
\gamma=\frac{\sqrt{2}}{2}
$$

implica na eliminação do termo $\alpha^{2}$ pertencente à expansão polinomial do denominador da equação (2.2), resultando numa forma notável que consiste exatamente no objetivo do filtro de Butterworth. Em verdade o filtro de Butterworth é uma escolha adequada dos coeficientes de uma equação diferencial para que sejam conservadas as amplitudes para certos valores da freqüência e anuladas outras. 


\section{Capítulo III}

\section{TRANSFORMADA DE FOURIER}

\subsection{INTRODUÇÃO}

A transformada de Fourier[3] é uma ferramenta matemática de grande utilidade no estudo das equações diferenciais, especialmente no caso das envolvidas no problema da filtragem de freqüências, tendo-se em vista que, no domínio transformado, uma equação diferencial na variável tempo converte-se numa equação algébrica no domínio da freqüência, de manipulação mais imediata.

No sentido de facilitar a exposição e o entendimento, inicia-se pela abordagem da série de Fourier, cujo desenvolvimento permite explicitar a formulação da transformada de Fourier, bem como sua formulação inversa. $\mathrm{Na}$ seqüência a propriedade da transformada de derivadas é estudada em maior detalhe, visando facilitar a análise das equações diferenciais, uma vez que para a transformada de Fourier vale o princípio da linearidade. A aplicação na equação de movimento, mostrada em seguida, exibe as etapas operacionais de maior interesse. 


\subsection{SÉRIE DE FOURIER}

Como sabido[3], uma função periódica $f(t)$ de período $T$, ou seja, $f(t)=f(t+T)$, pode ser desenvolvida segundo a série de Fourier:

$$
f(t)=\frac{b_{0}}{2}+\sum_{k=1}^{\infty}\left(a_{k} \operatorname{sen}(k \omega t)+b_{k} \cos (k \omega t)\right)
$$

onde:

$$
\begin{aligned}
& \omega=\frac{2 \pi}{T} \\
& a_{k}=\frac{\omega}{\pi} \int_{0}^{T} f(t) \operatorname{sen}(k \omega t) d t \\
& b_{k}=\frac{\omega}{\pi} \int_{0}^{T} f(t) \cos (k \omega t) d t \\
& b_{0}=\frac{\omega}{\pi} \int_{0}^{T} f(t) d t
\end{aligned}
$$

\subsection{TRANSFORMADA DE FOURIER}

A transformada da Fourier decorre da série de Fourier com a passagem ao limite do o período tendendo para infinito. Assim, $\omega$ como expresso na primeira de (3.2), tende para um diferencial $d \omega$. O termo fora do somatório em (3.1) tende para zero, e o somatório tende para a integral:

$$
f(t)=\int_{0}^{\infty}(A(\omega) \operatorname{sen}(\omega t)+B(\omega) \cos (\omega t)) d \omega
$$

onde:

$$
\begin{aligned}
& A(\omega)=\frac{1}{\pi} \int_{0}^{\infty} f(\tau) \operatorname{sen}(\omega \cdot \tau) d \tau \\
& B(\omega)=\frac{1}{\pi} \int_{0}^{\infty} f(\tau) \cos (\omega \cdot \tau) d \tau
\end{aligned}
$$


são conhecidas como integrais de Fourier.

A expressão (3.3) pode assumir a forma:

$$
f(t)=\int_{0}^{\infty}\left(\frac{1}{\pi} \int_{0}^{\infty} f(\tau)(\operatorname{sen}(\omega \tau) \operatorname{sen}(\omega t)+\cos (\omega \tau) \cos (\omega t)) d \tau\right) d \omega
$$

ou ainda, tendo-se em vista a propriedade trigonométrica da soma de ângulos:

$$
f(t)=\int_{0}^{\infty}\left(\frac{1}{\pi} \int_{-\omega}^{\infty} f(\tau) \cos (\omega(t-\tau)) d \omega\right) d \tau
$$

Por outro lado, sendo o cosseno uma função par e o seno uma função ímpar, pode-se escrever:

$$
\begin{aligned}
& \int_{-\infty}^{\infty} \mathrm{f}(\tau) \cos (\omega(\mathrm{t}-\tau)) \mathrm{d} \omega=2 \int_{0}^{\infty} \mathrm{f}(\tau) \cos (\omega(\mathrm{t}-\tau)) \mathrm{d} \omega \\
& \mathrm{i} \int_{-\infty}^{\infty} \mathrm{f}(\tau) \operatorname{sen}(\omega(\mathrm{t}-\tau)) \mathrm{d} \omega=0
\end{aligned}
$$

onde a multiplicação da segunda equação de (3.7) pela unidade complexa não altera, naturalmente, o resultado.

Somando-se membro a membro as equações (3.7) tem-se:

$$
\int_{-\infty}^{\infty} f(\tau) e^{i \omega(t-\tau)} d \omega=2 \int_{0}^{\infty} f(\tau) \cos (\omega(t-\tau)) d \omega
$$

o que permite reescrever (3.6) na forma:

$$
f(t)=\frac{1}{2 \pi} \int_{-\infty}^{\infty}\left(\int_{0}^{\infty} f(\tau) e^{-i \omega \tau} d \tau\right) e^{i \omega t} d \omega
$$

verificando-se, pois, de imediato que:

$$
\begin{aligned}
& F(\omega)=\int_{0}^{\infty} f(t) e^{-i \omega t} d t \\
& f(t)=\frac{1}{2 \pi} \int_{-\infty}^{\infty} F(\omega) e^{i \omega t} d \omega
\end{aligned}
$$


onde a primeira de (3.10) define a transformada de Fourier, e a segunda de (3.10) a sua correspondente inversa.

\subsection{PROPRIEDADE DA TRANSFORMADA DE INTERESSE NA RESOLUÇÃO DE EQUAÇÕES DIFERENCIAIS}

Inicialmente, demonstra-se o princípio da linearidade, fundamental para a aplicação da transformada em equações diferenciais. Assim, no caso de uma função $f(t)$ composta, por exemplo, por uma combinação linear entre duas funções $a(t)$ e $b(t)$, ou seja:

$$
f(t)=c_{1} a(t)+c_{2} b(t)
$$

onde $c_{1}$ e $c_{2}$ são constantes. Nesse caso, devido a transformação ser da forma de uma integração, onde a integral da soma é a soma das integrais, a transformada de $f(t)$ relaciona-se linearmente com as transformadas de $a(t)$ e de $b(t)$, como facilmente verifica-se nas passagens a seguir:

$$
\begin{aligned}
& F(\omega)=\int_{0}^{\infty} f(t) e^{-i \omega t} d t=\int_{0}^{\infty}\left(c_{1} a(t)+c_{2} b(t)\right) e^{-i \omega t} d t \\
& F(\omega)=c_{1} \int_{0}^{\infty} a(t) e^{-i \omega t} d t+c_{2} \int_{0}^{\infty} b(t) e^{-i \omega t} d t
\end{aligned}
$$

resultando:

$$
\mathrm{F}(\omega)=\mathrm{c}_{1} \mathrm{~A}(\omega)+\mathrm{c}_{2} \mathrm{~B}(\omega)
$$

Dentre as propriedades da transformada de Fourier, apresenta interesse mais direto no estudo da filtragem de freqüência a propriedade dita de derivação, a merecer um tratamento mais detalhado no que se segue. Seja transformada da derivada de função representada como sendo $\dot{F}(\omega)$, ou seja:

$$
\dot{\mathrm{F}}(\omega)=\int_{0}^{\infty} \frac{\mathrm{df}(\mathrm{t})}{\mathrm{dt}} \mathrm{e}^{-\mathrm{i} \omega \mathrm{t}} \mathrm{dt}
$$

onde o ponto superior na transformada apenas simboliza tratar-se da transformada da derivada. Por outro lado, a integração por partes de (3.14) permite explicitar: 


$$
\int_{0}^{\infty} \frac{d f}{d t}(t) e^{-i \omega t} d t=-\int_{0}^{\infty} f(t) \frac{\partial e^{-i \omega t}}{\partial t} d t+\left.\left(f(t) e^{-i \omega t}\right)\right|_{t=0} ^{t=\infty}
$$

Admitindo-se atendidas as condições de Dirichlet, ou seja, $\lim _{t \rightarrow \infty} f(t)=0$, tem-se finalmente que:

$$
\dot{\mathrm{F}}(\omega)=\mathrm{i} \omega \cdot \mathrm{F}(\omega)-\mathrm{f}(0)
$$

A transformada da segunda derivada, por conseguinte, pode ser redigida como:

$$
\ddot{F}(\omega)=(i \omega)^{2} F(\omega)-i \omega \cdot f(0)-\left.\frac{d f(t)}{d t}\right|_{t=0}
$$

uma vez que, por recorrência, a derivada segunda é, em verdade, a derivada da derivada primeira.

Como regra geral, pode-se provar que:

$$
\int_{0}^{\infty} \frac{d^{N} f(t)}{d t^{N}} e^{-i \omega t} d t=(i \omega)^{N} F(\omega)-\left.\sum_{j=0}^{N-1}(i \omega)^{j} \frac{d^{(N-j-1)} f(t)}{d t^{(N-j-1)}}\right|_{t=0}
$$

Nota-se que esta propriedade permite deixar as transformadas das derivadas de uma função $f(t)$ dependentes apenas de sua transformada $F(\omega)$ e das condições iniciais. Esta característica da Transformada de Fourier permite estudar equações diferenciais, se conhecidas suas condições iniciais.

\subsection{TRANSFORMADA DA FUNÇÃO SENOIDAL}

No sentido de estudar a transformada da função senoidal, ou seja:

$$
f(t)=\operatorname{sen}\left(\omega_{0} t\right)
$$

ou ainda em notação complexa:

$$
f(t)=\frac{e^{i \omega_{0} t}-e^{-i \omega_{0} t}}{2 i}
$$


torna-se necessário, por conveniência, formular a questão no sentido inverso, ou seja:

$$
\frac{1}{2 \pi} \int_{0}^{\infty} F(\omega) e^{i \omega t} d \omega=\frac{e^{i \omega_{0} t}-e^{-i \omega_{0} t}}{2 i}
$$

resultando:

$$
\mathrm{F}(\omega)=\frac{\pi}{\mathrm{i}}\left(\delta\left(\omega_{0}\right)-\delta\left(-\omega_{0}\right)\right)
$$

onde se emprega a clássica função delta de Dirac nas posições $-\omega_{0}$ e $+\omega_{0}$, porquanto, verifica-se facilmente que:

$$
\frac{1}{2 \pi} \int_{-\infty}^{\infty}\left[\frac{\pi}{\mathrm{i}}\left(\delta\left(\omega_{0}\right)-\delta\left(-\omega_{0}\right)\right)\right] \mathrm{e}^{\mathrm{i} \omega \mathrm{t}} \mathrm{d} \omega=\frac{1}{2 \mathrm{i}} \mathrm{e}^{\mathrm{i} \omega_{0} \mathrm{t}}-\frac{1}{2 \mathrm{i}} \mathrm{e}^{-\mathrm{i} \omega_{0} \mathrm{t}}
$$

A figura 3.1a mostra esquematicamente a transformada de Fourier da função senoidal e a figura $3.1 b$ a transformada da função cossenoidal obtida de maneira similar.
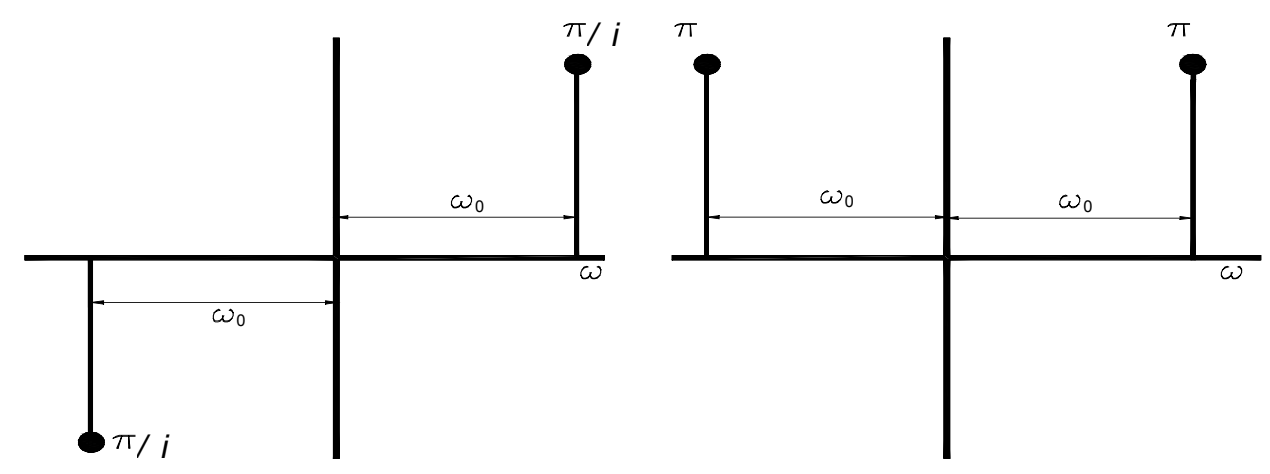

Fig. 3.1 - Trasformada de Fourier de seno e cosseno respectivamente

\subsection{APLICAÇÃO NA EQUAÇÃO DE MOVIMENTO}

No sentido de mostrar a aplicação da transformada de Fourier na solução de equações diferenciais lineares, retome-se o caso da equação de movimento considerando-se carregamento senoidal, ou seja:

$$
\ddot{\rho}(\mathrm{t})+2 \gamma \omega_{n} \dot{\rho}(t)+\omega_{n}^{2} \rho(t)=\omega_{n}^{2} \operatorname{sen}\left(\omega_{0} t\right)
$$


Tomando-se a transformada de Fourier em ambos os membros de (3.24), tem-se:

$$
(i \omega)^{2} \rho(\omega)+2 \gamma \omega_{n}(i \omega) \rho(\omega)+\omega_{n}^{2} \rho(\omega)=\omega_{n}^{2}\left[i \pi\left(\delta\left(-\omega_{0}\right)-\delta\left(\omega_{0}\right)\right)\right]
$$

o que permite explicitar a solução no domínio da freqüência como:

$$
\rho(\omega)=\frac{i \pi\left(\delta\left(-\omega_{0}\right)-\delta\left(\omega_{0}\right)\right)}{1-\alpha^{2}+i 2 \gamma \alpha}
$$

Por outro lado, procedendo-se a transformação inversa tem-se:

$$
\rho(t)=\frac{1}{2 \pi} \int_{-\infty}^{\infty} \frac{i \pi\left(\delta\left(-\omega_{0}\right)-\delta\left(\omega_{0}\right)\right)}{1-\alpha^{2}+i 2 \gamma \alpha} e^{i \omega t} d \omega
$$

que resulta em:

$$
\rho(t)=\frac{1}{2 \pi}\left[\frac{i \pi}{1-\alpha^{2}+i 2 \gamma \alpha} e^{-i \omega_{0} t}-\frac{i \pi}{1-\alpha^{2}+i 2 \gamma \alpha} e^{i \omega_{0} t}\right]
$$

ou ainda, transpondo (3.28) para a forma trigonométrica:

$$
\rho(t)=\frac{A}{\sqrt{\left(1-\alpha^{2}\right)^{2}+\left(2 \gamma \alpha_{0}\right)^{2}}} \operatorname{sen}\left(\omega_{0} t-\varphi\right)
$$

onde operações no domínio complexo de alguma monta foram necessárias, sendo que:

$$
\varphi=\arctan \left(\frac{2 \gamma \alpha}{1-\alpha^{2}}\right)
$$

encerrando-se assim a aplicação da transformada na equação de movimento.

\subsection{APLICAÇÃO DA TRANSFORMADA EM UMA EQUAÇÃO DIFERENCIAL DE ORDEM GENÉRICA}

Uma equação diferencial ordinária pode ser generalizada como sendo uma igualdade entre uma função de entrada $x(t)$ no segundo membro e um somatório 
de $\mathrm{N}$ termos no primeiro membro envolvendo derivadas de uma função de saída $y(t)$, ou incógnita, ou seja:

$$
\sum_{k=0}^{N} a_{k} \frac{d^{k} y(t)}{d t^{k}}=x(t)
$$

onde os termos $a_{k}$ são constantes e o expoente $k$ nas derivadas indica o grau de derivação.

Tomando-se a transformada de Fourier em ambos os membros de (3.31) tem-se:

$$
\sum_{k=0}^{N} a_{k}\left[(i \omega)^{k} Y(\omega)-\left.\sum_{j=0}^{k-1}(i \omega)^{j} \frac{d^{(k-j-1)} y(t)}{d t^{(k-j-1)}}\right|_{t=0}\right]=X(\omega)
$$

Onde por outro lado, sendo as condições iniciais nulas, a igualdade (3.32) torna-se:

$$
\mathrm{Y}(\omega) \sum_{\mathrm{k}=0}^{\mathrm{N}} \mathrm{a}_{\mathrm{k}}(\mathrm{i} \omega)^{\mathrm{k}}=\mathrm{X}(\omega)
$$

e, nesse caso, a relação existente entre $Y(\omega)$ e $X(\omega)$ escreve-se:

$$
H(\omega)=\frac{Y(\omega)}{X(\omega)}
$$

onde $H(\omega)$ é a denominada função de resposta em freqüência, e expressa por:

$$
H(\omega)=\frac{1}{\sum_{k=0}^{N} a_{k}(i \omega)^{k}}
$$

encerrando-se dessa forma o procedimento necessário para transformar uma equação diferencial no domínio do tempo para uma equação algébrica no domínio da freqüência. 


\section{Capítulo IV}

\section{EQUAÇÃO DE FILTRAGEM}

\subsection{INTRODUÇÃO}

O procedimento analítico envolvido na filtragem de freqüências segundo o modelo de Butterworth é o objeto do presente capítulo. Inicia-se apresentando as idéias básicas da filtragem de freqüências, discutindo-se os filtros do tipo passabaixa, e os filtros dele derivados, como o passa-alta, o passa-banda, e o rejeitabanda.

Como já mostrado no capítulo III, a integração de equações diferenciais lineares a coeficientes constantes no domínio da freqüência resume-se numa operação algébrica de multiplicação da transformada de Fourier do sinal de entrada (carregamento no caso da equação de movimento) pela função de resposta em freqüência, que depende dos coeficientes da equação diferencial. Assim sendo, o problema da filtragem de freqüência, como se pode perceber, é governado basicamente pela função de resposta em freqüência $H(\omega)$.

O modelo de filtragem de Butterworth consiste essencialmente em se buscar uma forma adequada para o módulo da função de resposta em freqüência, e conseqüente escolha apropriada dos parâmetros da equação diferencial correspondente, e com ordens variáveis, de modo a conferir um grau de eficiência crescente ao processo de filtragem. A literatura especializada nesse tema, via de 
regra, apresenta o assunto por meio da transformada de Laplace, e não pela transformada de Fourier aqui considerada. Todavia, tal escolha se deve ao fato de que, em dinâmica estrutural, assunto de maior domínio para os engenheiros civis, a transformada de Fourier é, sem dúvida, bem mais empregada e trabalhada.

\subsection{CONCEITUAÇÃO DE FILTRO}

A idéia básica do filtro de freqüências está associada naturalmente a um dispositivo que retém algo indesejado, como no caso mais conhecido de uma peneira, deixando-se passar algo que se deseja. Assim sendo, no caso de um sinal genérico contendo componentes de diversas freqüências, o que pode ser entendido como uma sobreposição de sinais harmônicos, sua filtragem no domínio da freqüência mediante filtro passa-baixa, por exemplo, consiste na eliminação das componentes de freqüência acima de uma especificada, normalmente referida como freqüência de corte.

No domínio da freqüência, como já referido no capítulo anterior, denominase $X(\omega)$ a transformada de Fourier do sinal de entrada e $Y(\omega)$ a transformada de Fourier do sinal de saída, ou seja, do sinal filtrado. Para se proceder ao exame do processo de filtragem, retome-se a função de resposta em freqüência $H(\omega)$, que consiste na relação entre $\mathrm{Y}(\omega) / \mathrm{X}(\omega)$, como visto na equação (3.34). Colocando-se então a relação (3.34) na forma:

$$
Y(\omega)=H(\omega) X(\omega)
$$

Notando-se em primeira vista que, numa situação ideal, quando se tem $H(\omega)=1$ conserva-se integralmente na resposta o correspondente sinal de entrada (o filtro não retém nada), e que quando $H(\omega)=0$ anula-se o sinal de resposta (o filtro não deixa passar nada). Todavia, essa forma ideal para $H(\omega)$ não dispõe de uma equação diferencial correspondente no domínio do tempo ou freqüência.

Por outro lado, nos casos práticos, tem-se, em geral, uma relação entre o sinal de entrada e o de saída no domínio do tempo dada por uma equação diferencial, cuja transformada de Fourier implica numa função $H(\omega)$ complexa como expressa na forma (3.35). Assim, considerando-se que $H(\omega)$ e $X(\omega)$ sejam complexos, em notação polar a equação (4.1) permite escrever: 


$$
\begin{aligned}
& |Y(\omega)|=|H(\omega)||X(\omega)| \\
& \arg [Y(\omega)]=\arg [H(\omega)]+\arg [X(\omega)]
\end{aligned}
$$

onde o módulo da resposta resulta do produto do módulo do sinal de entrada pelo módulo da função de resposta em freqüência, e o argumento da resposta (ângulo no plano de Gauss) resulta na soma dos argumentos (emprega-se em (4.2) a notação clássica de módulo entre barras verticais).

Uma vez que se procura na filtragem conservar a amplitude do sinal desejado, isso implica naturalmente em se ter o módulo $|H(\omega)|$ próximo da unidade para a faixa de valores desejados de freqüência, e também que o módulo $|H(\omega)|$ seja nulo em caso contrário.

\subsection{TIPOS DE FILTRO}

A figura 4.1 exibe em situação ideal os quatro tipos de filtro de maior interesse. O filtro passa-baixa é aquele em que somente as componentes com freqüências abaixo de um certo valor da freqüência de corte são coletadas (Fig.4.1.1). De maneira similar, o filtro passa-alta retém as componentes com freqüências abaixo da freqüência de corte e conserva as com freqüências acima (Fig.4.1.2). O filtro passa-banda coleta apenas as componentes com freqüências dentro de uma faixa (Fig.4.1.3). O último tipo de filtro é o denominado elimina-banda, que exclui apenas as componentes com freqüências numa determinada faixa (Fig.4.1.4).

É fácil verificar que os filtros passa-alta, passa-banda e elimina-banda podem ser obtidos em função do filtro passa-baixa. Por exemplo, o filtro passaalta opera como se o sinal de entrada fosse subtraído do sinal de saída do filtro passa-baixa, o passa-banda resulta da aplicação de um filtro passa-alta com $\omega=\omega_{\mathrm{p}}$, e sobre o resultado aplicando-se um filtro passa-baixa com $\omega=\omega_{\mathrm{c}}, 0$ rejeita-banda opera como se subtraísse do sinal de entrada o resultado de um filtro passa-banda. 


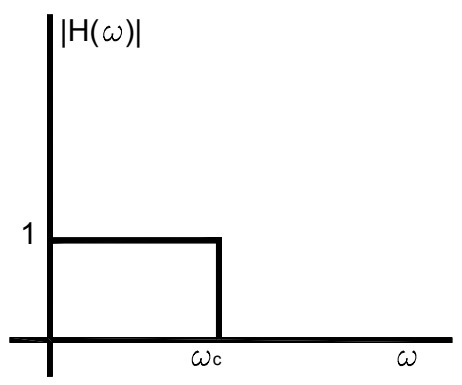

Fig. 4.1.1. - Passa-baixa

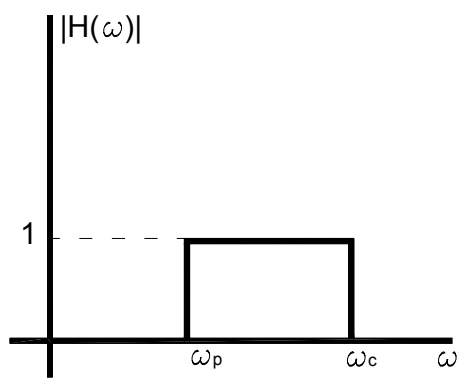

Fig. 4.1.3. - Passa-banda

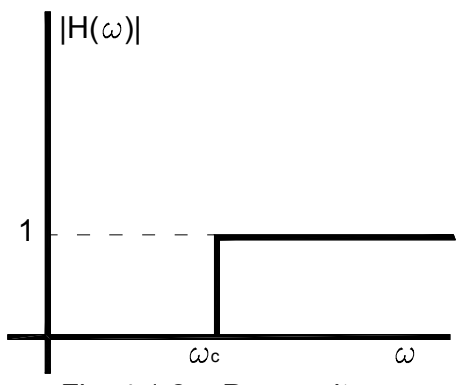

Fig. 4.1.2. - Passa-alta

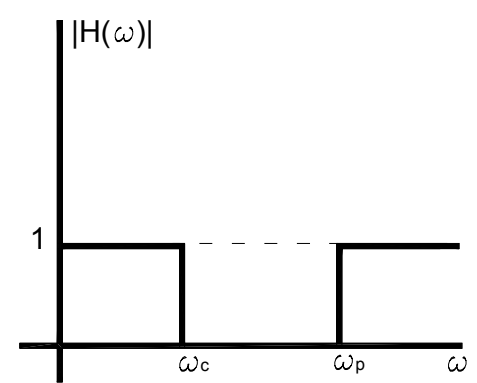

Fig. 4.1.4. -

Fig. 4.1 - Tipos básicos de filtros

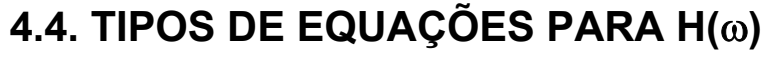

Um filtro passa-baixa ideal seria aquele onde $|H(\omega)|=1$ para freqüências abaixo da freqüência de corte $\left(\omega<\omega_{c}\right)$ e $|H(\omega)|=0$ para as demais freqüências, ou seja $\left(\omega>\omega_{c}\right)$. Todavia, vale registrar que um primeiro inconveniente dessa situação ideal seria o fato de que tais condições não seriam realizáveis do ponto de vista analítico, dada a descontinuidade no ponto $\omega=\omega_{c}$. A literatura oferece três tipos de equações práticas para $H(\omega)$. $O$

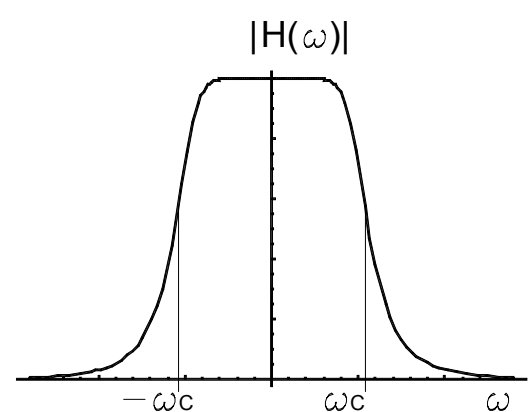

Fig. 4.2.1. - Filtro de Butterworth

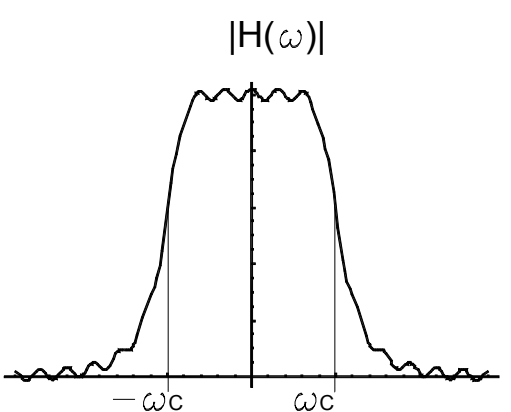

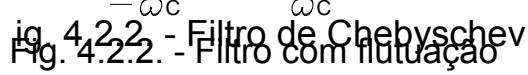

primeiro, mais conhecido, é o chamado filtro de Butterworth (Fig.4.2.1), que será examinado neste trabalho, e que é uma aproximação polinomial com apenas um ponto de inflexão em $\omega_{\mathrm{c}}$. Os outros dois tipos são o Chebyschev e o 
Elíptico, chamados filtros com flutuação (Fig.4.2.2), que apresentam formas parecidas onde uma pequena oscilação está presente ao longo de $|H(\omega)|$. A vantagem destes dois últimos tipos é que o corte apresenta zona de transição mais abrupta no ponto $\omega_{c}$, aproximando-se, nesse sentido, mais do filtro ideal.

$\mathrm{Na}$ literatura o filtro de Butterworth é tratado por meio da transformada de Laplace, que é bastante similar à transformada de Fourier, exceto pelo fato de lidar com exponenciação com valores reais, e não complexos.

\subsection{FUNÇÃO $H(\omega)$}

Retomando-se o resultado exposto no final do estudo das equações diferenciais levado a efeito no capítulo III, tem-se a seguinte redação para a função de resposta em freqüência $H(\omega)$ :

$$
H(\omega)=\frac{1}{\sum_{\mathrm{k}=0}^{\mathrm{N}} \mathrm{a}_{\mathrm{k}}(\mathrm{i} \omega)^{\mathrm{k}}}
$$

sendo $\mathrm{N}$ o grau da equação diferencial correspondente, que também é chamado de número de pólos do filtro, como adiante esclarecido.

\subsection{APLICAÇÃO PARA UMA ENTRADA SENOIDAL}

Considerando-se que o sinal de entrada é uma senóide (sinal harmônico simples), ou seja:

$$
x(t)=A \cdot \operatorname{sen}\left(\omega_{0} t\right)
$$

tem-se, como mostrado no capítulo precedente, que sua transformada de Fourier assim se expressa:

$$
\mathrm{X}(\omega)=-\mathrm{i} \mathrm{A} \pi\left(\delta\left(\omega_{0}\right)-\delta\left(-\omega_{0}\right)\right)
$$

e, nesse caso, o expresso em (4.1) fica:

$$
\mathrm{Y}(\omega)=\mathrm{H}(\omega)\left[-\mathrm{iA} \pi\left(\delta\left(\omega_{0}\right)-\delta\left(-\omega_{0}\right)\right)\right]
$$

ou ainda, na forma inversa: 


$$
y(t)=\frac{1}{2 \pi} \int_{-\infty}^{\infty} H(\omega)\left[-i A \pi\left(\delta\left(\omega_{0}\right)-\delta\left(-\omega_{0}\right)\right)\right] e^{i \omega t} d \omega
$$

Por outro lado, tendo-se em vista que a função delta de Dirac faz com que a integral se reduza ao $\mathrm{d} \omega$ do ponto $\omega_{0}$, tem-se:

$$
y(t)=\frac{A}{2 i}\left(H\left(\omega_{0}\right) e^{i \omega_{0} t}-H\left(-\omega_{0}\right) e^{-i \omega_{0} t}\right)
$$

onde, dispondo na forma trigonométrica os exponenciais e providenciando-se conveniente fatoração chega-se à:

$$
y(t)=\frac{A}{2 i}\left[\cos \left(\omega_{0} t\right)\left(H\left(\omega_{0}\right)-H\left(-\omega_{0}\right)\right)+i \cdot \operatorname{sen}\left(\omega_{0} t\right)\left(H\left(\omega_{0}\right)+H\left(-\omega_{0}\right)\right)\right]
$$

onde:

$$
\begin{gathered}
H\left(\omega_{0}\right)=\frac{1}{\sum_{\mathrm{k}=0}^{\mathrm{N}} \mathrm{a}_{\mathrm{k}}\left(\mathrm{i} \omega_{0}\right)^{\mathrm{k}}} \\
\mathrm{H}\left(-\omega_{0}\right)=\frac{1}{\sum_{\mathrm{k}=0}^{\mathrm{N}} \mathrm{a}_{\mathrm{k}}\left(-\mathrm{i} \omega_{0}\right)^{\mathrm{k}}}
\end{gathered}
$$

que, em princípio, têm forma complexa. Pode-se notar que o somatório no denominador tem valores reais e imaginários. A parte real das duas formas é sempre igual para valores pares do fator $k$, e a parte imaginária terá sinal oposto nos casos de k ser ímpar. Dispondo de uma forma mais confortável, $H\left(\omega_{0}\right)$ e $H\left(-\omega_{0}\right)$ podem ser escritos como:

$$
\begin{aligned}
& H\left(\omega_{0}\right)=\frac{1}{R e+i \operatorname{Im}}=\frac{R e-i I m}{R^{2}+I^{2}} \\
& H\left(-\omega_{0}\right)=\frac{1}{R e-i \operatorname{Im}}=\frac{R e+i \operatorname{Im}}{R^{2}+\operatorname{Im}^{2}}
\end{aligned}
$$

onde o argumento de $\mathrm{H}\left(\omega_{0}\right)$ se expressa:

$$
\arg \left[\mathrm{H}\left(\omega_{0}\right)\right]=\arctan \left(-\frac{\operatorname{Im}}{\operatorname{Re}}\right)
$$


sendo o módulo dado por:

$$
\left|\mathrm{H}\left( \pm \omega_{0}\right)\right|=\frac{1}{\sqrt{\mathrm{Re}^{2}+\mathrm{Im}^{2}}}
$$

verificando-se assim uma identidade esperada.

Assim sendo, fazendo-se as operações indicadas em (4.9) para $H(\omega)$, que consiste na soma e subtração dos valores em (4.11), tem-se:

$$
\begin{aligned}
& H\left(\omega_{0}\right)+H\left(-\omega_{0}\right)=\frac{2 \mathrm{Re}}{\mathrm{Re}^{2}+\mathrm{Im}^{2}} \\
& H\left(\omega_{0}\right)-H\left(-\omega_{0}\right)=\frac{-2 \mathrm{i} I \mathrm{Im}}{\mathrm{Re}^{2}+\mathrm{Im}^{2}}
\end{aligned}
$$

e, denominando-se $\theta$ o argumento de $H\left(\omega_{0}\right)$, pode-se escrever:

$$
\begin{gathered}
\operatorname{sen}(\theta)=-\frac{\operatorname{Im}}{\sqrt{\mathrm{Re}^{2}+\mathrm{Im}^{2}}} \\
\cos (\theta)=\frac{\mathrm{Re}}{\sqrt{\mathrm{Re}^{2}+\mathrm{Im}^{2}}}
\end{gathered}
$$

Com isso, uma nova redação pode ser alcançada:

$$
\begin{aligned}
& H\left(\omega_{0}\right)+H\left(-\omega_{0}\right)=2\left|H\left(\omega_{0}\right)\right| \cos (\theta) \\
& H\left(\omega_{0}\right)-H\left(-\omega_{0}\right)=2 i\left|H\left(\omega_{0}\right)\right| \operatorname{sen}(\theta)
\end{aligned}
$$

deixando-se evidente que a soma resulta real e a subtração resulta complexo puro.

Finalmente, substituindo os valores (4.16) em (4.9) chega-se à forma:

$$
y(t)=A\left|H\left(\omega_{0}\right)\right|\left[\operatorname{sen}\left(\omega_{0} t\right) \cos (\theta)+\cos \left(\omega_{0} t\right) \operatorname{sen}(\theta)\right]
$$

e, reescrevendo a igualdade 4.17 de uma forma mais conveniente, ou seja, empregando-se a notação senoidal, tem-se:

$$
y(t)=A\left|H\left(\omega_{0}\right)\right| \operatorname{sen}\left(\omega_{0} t+\arg \left[H\left(\omega_{0}\right)\right]\right)
$$

encerrando-se a formulação de interesse, mostrando-se que a resposta tem amplitude dada pelo produto da amplitude da entrada pelo módulo da função 
de resposta em freqüência, e também que há uma defasagem dada pelo argumento da função de resposta em freqüência.

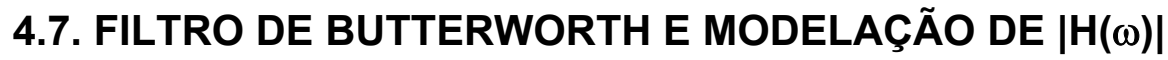

Conforme se constata de (4.18), a eficiência da filtragem em freqüência decorre de uma adequada escolha para o módulo $|H(\omega)|$. No modelo de Butterworth tal escolha é do tipo:

$$
\left|\mathrm{H}_{\mathrm{N}}(\omega)\right|=\frac{1}{\sqrt{1+\left(\frac{\omega}{\omega_{\mathrm{c}}}\right)^{2 \mathrm{~N}}}}
$$

onde $\mathrm{N}$ é o número de pólos correspondente, como mais adiante explicado. $\mathrm{A}$ figura 4.3 exibe, a título de visualização, o comportamento numérico do módulo

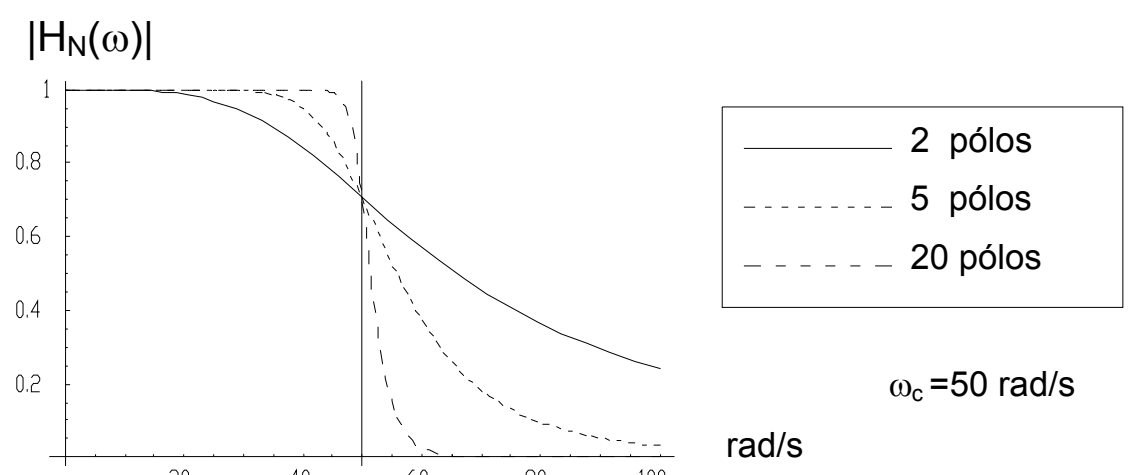

Fig. 4.3 - Comportamento de (4.19) para $\mathrm{N}$ assumindo valores de 2, 5 e 20

da função de resposta em freqüência como expresso em (4.19) para os casos de 2, 5 e 20 pólos, mostrando o andamento assintótico para o comportamento do chamado filtro ideal. Deve-se ressaltar que o módulo da função de resposta em freqüência assume o valor $\sqrt{2} / 2$ para $\omega=\omega_{c}$, ou seja, na freqüência de corte.

A questão básica que se coloca consiste em se determinar a equação diferencial cuja função de resposta em freqüência proporciona a expressão (4.19) para $|H(\omega)|$. Nesse sentido, vale reproduzir a igualdade 4.3, separando-se a parte real da imaginária de $H(\omega)$, que no caso de $N$ par resulta: 


$$
H_{\text {par }}(\omega)=\frac{1}{\underbrace{\sum_{\mathrm{k}=0}^{\frac{\mathrm{N}}{2}} \mathrm{a}_{2 \mathrm{k}}(\mathrm{i} \omega)^{2 \mathrm{k}}}_{\text {Real }}+\underbrace{\sum_{\mathrm{k}=0}^{\frac{\mathrm{N}}{2}} \mathrm{a}_{2 \mathrm{k}+1}(\mathrm{i} \omega)^{2 \mathrm{k}+1}}_{\text {Im aginário }}}
$$

e cujo módulo vale

$$
\left|\mathrm{H}_{\mathrm{par}}(\omega)\right|=\frac{1}{\sqrt{\left[\sum_{\mathrm{k}=0}^{\frac{\mathrm{N}}{2}} \mathrm{a}_{2 \mathrm{k}}(-1)^{\mathrm{k}} \omega^{2 \mathrm{k}}\right]^{2}+\left[\sum_{\mathrm{k}=0}^{\frac{\mathrm{N}}{2}} \mathrm{a}_{2 \mathrm{k}-1} \omega^{2 \mathrm{k}-1}(-1)^{\mathrm{k}-1}\right]^{2}}}
$$

sendo que no radical a maior potência de $\omega$ é dada por $2 \mathrm{~N}$. No caso de $\mathrm{N}$ ímpar tem-se:

$$
\left|\mathrm{H}_{\text {impar }}(\omega)\right|=\frac{1}{\sqrt{\left[\sum_{\mathrm{k}=0}^{\frac{\mathrm{N}-1}{2}} \mathrm{a}_{2 \mathrm{k}}(-1)^{\mathrm{k}} \omega^{2 \mathrm{k}}\right]^{2}+\left[\sum_{\mathrm{k}=0}^{\frac{\mathrm{N}-1}{2}} \mathrm{a}_{2 \mathrm{k}+1} \omega^{2 \mathrm{k}+1}(-1)^{\mathrm{k}}\right]^{2}}}
$$

novamente concluindo-se que $2 \mathrm{~N}$ é o maior grau do polinômio dentro da raiz quadrada.

Assim sendo, a questão agora é encontrar apropriados parâmetros $a_{k}$ em (4.21) e (4.22) de modo a resultar a forma desejada (4.19). Essa é a tarefa tratada no que se segue.

\subsection{ESCOLHA DOS PARÂMETROS}

A escolha adequada dos parâmetros $a_{k}$ em (4.3) de modo a resultar em (4.19) é bastante facilitada tendo-se em vista que é possível encontrar uma forma fatorada para o termo na raiz quadrada em (4.19), bastando para tanto encontrar, como bem conhecido da teoria da equações algébricas, as raízes de:

$$
1+\left(\frac{\omega}{\omega_{c}}\right)^{2 \mathrm{~N}}=0
$$


ou seja:

$$
\left(\frac{\omega}{\omega_{c}}\right)^{2 N}=-1
$$

que consiste em se buscar as $2 \mathrm{~N}$ raízes da unidade negativa.

No sentido de abreviar a exposição, chamando-se:

$$
\mathrm{z}=\frac{\omega}{\omega_{\mathrm{c}}}
$$

é fácil verificar, como ilustrado no plano de Gauss exibido na figura 4.4, que as raízes equação algébrica (4.24) são dadas por:

$$
z^{2 N}=-1
$$

ou ainda em forma polar, admitindo que o número complexo tem módulo unitário:

$$
\left(e^{i \phi}\right)^{2 N}=\cos (2 N \phi)+i \operatorname{sen}(2 N \phi)=-1
$$

cujas raízes são dadas então por:

$$
\begin{aligned}
& \cos (2 \mathrm{~N} \phi)=-1 \\
& \operatorname{sen}(2 \mathrm{~N} \phi)=0
\end{aligned}
$$

ou seja:

$$
\phi_{\mathrm{k}}=\frac{\pi+2 \mathrm{k} \pi}{2 \mathrm{~N}}
$$

com $\mathrm{k}=0,1, \ldots \ldots, 2 \mathrm{~N}-1$, e cuja ilustração gráfica das $2 \mathrm{~N}$ raízes em questão, ou também referidas como pólos na literatura clássica, indica-se na Fig. 4.4. 


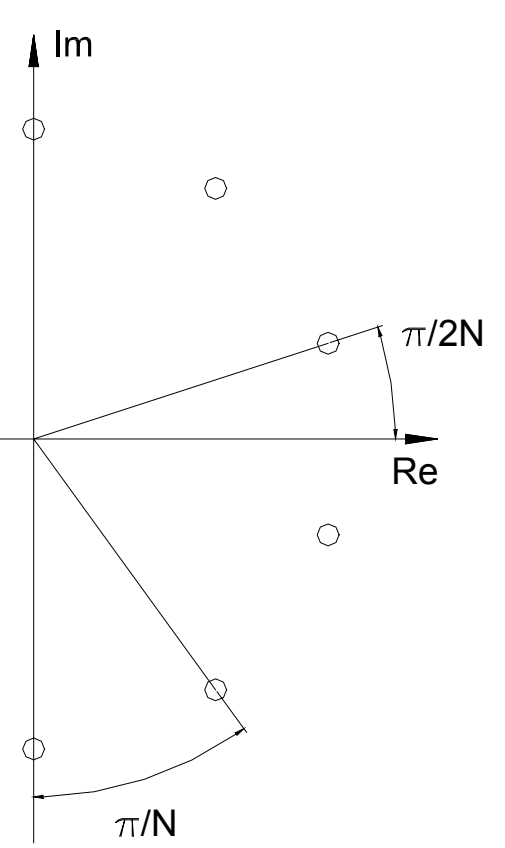

Fig. 4.4 - Raízes da equação 4.23

É oportuno assinalar que o fato de se considerar no desenvolvimento apresentado a transformada de Fourier, e não a transformada de Laplace, como mais trabalhada na literatura especializada, facilitou bastante o estudo das raízes, pois não se dependeu do fato de o parâmetro N ser par ou ímpar.

Para finalizar, cumpre, por oportuno, registrar pois a igualdade:

$$
1+\left(\frac{\omega}{\omega_{\mathrm{c}}}\right)^{2 \mathrm{~N}}=\prod_{\mathrm{k}=1}^{2 \mathrm{~N}}\left(\frac{\omega}{\omega_{\mathrm{c}}}-\left(\frac{\omega}{\omega_{\mathrm{c}}}\right)_{\mathrm{k}}\right)
$$

onde:

$$
\left(\frac{\omega}{\omega_{c}}\right)_{k}=e^{i \frac{\pi+2 k \pi}{2 N}}
$$

como sabido da teoria das equações algébricas.

Novamente lembra-se que neste trabalho procura-se evitar a utilização da Transformada de Laplace por esta ser incomum para os engenheiros de estruturas. Porém, a título de ilustração, observa-se que os pólos do polinômio de Butterworth em $s\left(B_{N}(s)\right)$ devem possuir parte real negativa e ainda não nula. 


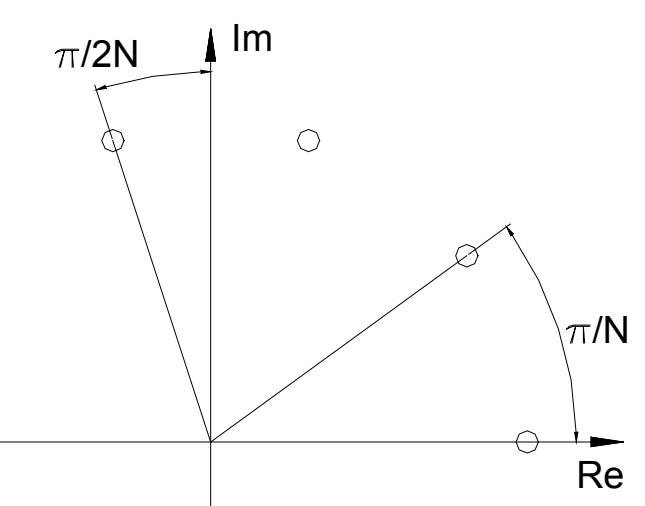

Fig. 4.5 - Raízes de $\left|\mathrm{B}_{\mathrm{N}}(\mathrm{s})\right|^{2}$

\subsection{ESTUDO DOS PÓLOS}

No sentido de facilitar a exposição, é conveniente verificar que o polinômio no denominador de (4.3), doravante denominado $\mathrm{P}_{\mathrm{N}}(\omega)$, ou seja:

$$
\mathrm{P}_{\mathrm{N}}(\omega)=\sum_{\mathrm{k}=0}^{\mathrm{N}} \mathrm{a}_{\mathrm{k}}(\mathrm{i} \omega)^{\mathrm{k}}
$$

e sua forma conjugada:

$$
\mathrm{P}_{\mathrm{N}}(-\omega)=\sum_{\mathrm{k}=0}^{\mathrm{N}} \mathrm{a}_{\mathrm{k}}(-\mathrm{i} \omega)^{\mathrm{k}}
$$

permitem explicitar a seguinte relação:

$$
\mathrm{P}_{\mathrm{N}}(\omega) \mathrm{P}_{\mathrm{N}}(-\omega)=\left|\mathrm{P}_{\mathrm{N}}(\omega)\right|^{2}=1+\left(\frac{\omega}{\omega_{\mathrm{c}}}\right)^{2 \mathrm{~N}}
$$

o que indica ser necessário para a definição dos coeficientes de $P_{N}(\omega)$, ou seja, os coeficientes da equação diferencial correspondente, apenas $\mathrm{N}$ raízes de (4.23). A escolha apropriada das $\mathrm{N}$ raízes a serem consideradas é objeto do que se segue. 
Em primeiro lugar, a equação diferencial correspondente deve, para que a filtragem seja eficiente, apresentar solução homogênea transitória, ou seja, deve contemplar apenas termos afetados por expoentes negativos, a exemplo de (2.30), de sorte a abreviar o resultado da filtragem. No caso instável, com expoentes positivos, a solução homogênea não permite a realização de filtragem, naturalmente. Para melhor explicar essa questão, inicia-se considerando a forma homogênea da equação diferencial (3.31), ou seja:

$$
\sum_{k=0}^{N} a_{k} \frac{d^{k} y(t)}{d t^{k}}=0
$$

e sua solução homogênea que é do tipo:

$$
y(t)=A e^{\lambda t}
$$

resultando, pois:

$$
\sum_{\mathrm{k}=0}^{\mathrm{N}} \mathrm{a}_{\mathrm{k}} \lambda^{\mathrm{k}}=0
$$

o que leva às mesmas raízes do polinômio $P_{N}(\omega)$. Assim sendo, para haver solução homogênea estável é necessário que o auto valor $\lambda$ não tenha parte real positiva, pois:

$$
e^{\lambda t}=e^{((a+i b) t)}=e^{a t} e^{i b t}
$$

completando-se desse modo a explicação, uma vez que o fator com exponencial complexo é retratado apenas por funções harmônicas.

Como o polinômio $P_{N}(\omega)$ contempla apenas $N$ raízes complexas $\left(\omega / \omega_{c}\right)_{k}$, tem-se, da teoria das equações algébricas, que:

$$
\mathrm{P}_{\mathrm{N}}(\omega)=\prod_{\text {Arranjo }}\left(\mathrm{i} \frac{\omega}{\omega_{\mathrm{c}}}-\mathrm{e}^{\mathrm{i}\left(\frac{\pi}{2 \mathrm{~N}}+\mathrm{k} \frac{\pi}{\mathrm{N}}+\frac{\pi}{2}\right)}\right)
$$

acarretando que a parcela $e^{\mathrm{i} \cdot\left(\frac{\pi}{2 \mathrm{~N}}+\mathrm{k} \frac{\pi}{\mathrm{N}}+\frac{\pi}{2}\right)}$ de (4.39) é que deve, naturalmente, ter parte real negativa, implicando-se, pois: 


$$
\cos \left(\frac{\pi}{2 \mathrm{~N}}+\mathrm{k} \frac{\pi}{\mathrm{N}}+\frac{\pi}{2}\right)<0
$$

o que conduz à inequação:

$$
\frac{\pi}{2}<\frac{\pi}{2 \mathrm{~N}}+\mathrm{k} \frac{\pi}{\mathrm{N}}+\frac{\pi}{2}<\frac{3 \pi}{2}
$$

cuja solução resulta:

$$
-\frac{1}{2}<\mathrm{k}<\mathrm{N}-\frac{1}{2} \longrightarrow 0<\mathrm{k}<\mathrm{N}-1
$$

podendo-se dizer então que as raízes do polinômio que contemplam a estabilidade, devem ser aquelas do lado superior do eixo das abscissas no plano de Gauss. Cumpre nesse ponto esclarecer que os pólos ditos de Butterworth são aqueles pertencentes ao lado esquerdo do eixo de ordenadas no plano de Gauss (parte real negativa), uma vez que ao se trabalhar com a transformada de Laplace tem-se uma substituição do tipo $s=i \omega$, sendo $s$ a variável da transformação no caso. Então, os pólos em s aparecem com uma defasagem de $+90^{\circ}$ em relação aos pólos dispostos no presente estudo no domínio da freqüência $\omega$. Chamando-se os pólos do hemisfério superior de $\mathrm{p}_{\mathrm{hs}}$, o expresso em (4.39) pode então ser escrito como:

$$
\mathrm{P}_{\mathrm{N}}(\omega)=\prod\left(\frac{\mathrm{i} \omega}{\omega_{\mathrm{c}}}-\mathrm{i} \cdot \mathrm{p}_{\mathrm{hs}}\right)
$$

encerrando-se assim esse estudo; cabendo-se ainda ressaltar que, como já comentado, a vantagem de se analisar estas raízes segundo a transformada de Fourier reside no fato de que os pólos assumem uma função fixa, não dependendo do fato de ser par ou ímpar o número de pólos.

\subsection{FILTROS E EQUAÇÕES DIFERENCIAIS CORRESPONDENTES}

Considerando-se o caso mais simples, ou seja, o caso $\mathrm{N}=1$, que consiste no filtro de um pólo, tem-se: 


$$
\mathrm{P}_{1}(\omega)=\prod_{\mathrm{k}=0}^{1-1}\left(\frac{\mathrm{i} \omega}{\omega_{\mathrm{c}}}-\mathrm{e}^{\mathrm{i} \cdot\left(\frac{\pi}{2}+\mathrm{k} \frac{\pi}{1}+\frac{\pi}{2}\right)}\right)=\frac{\mathrm{i} \omega}{\omega_{\mathrm{c}}}-\mathrm{e}^{\mathrm{i} \pi}=\frac{\mathrm{i} \omega}{\omega_{\mathrm{c}}}+1=\mathrm{a}_{1} \mathrm{i} \omega+\mathrm{a}_{0}
$$

resultando pois:

$$
a_{0}=1 \quad ; \quad a_{1}=\frac{1}{\omega_{c}}
$$

correspondendo-se à função de resposta em freqüência:

$$
H_{1}(\omega)=\frac{1}{\frac{\omega}{\omega_{c}} i+1}
$$

cujo módulo resulta:

$$
\left|H_{1}(\omega)\right|=\frac{1}{\sqrt{1+\left(\frac{\omega}{\omega_{c}}\right)^{2}}}
$$

exibindo-se a filtragem mais elementar.

A equação diferencial correspondente fica então com a seguinte escrita:

$$
\frac{1}{\omega_{c}} \frac{d y(t)}{d t}+y(t)=x(t)
$$

encerrando-se assim, no que interessa, o algoritmo de filtragem de um pólo.

Considerando-se agora o caso de dois pólos, tem-se:

$$
P_{2}(\omega)=\left(\frac{i \omega}{\omega_{c}}-e^{i \frac{3 \pi}{4}}\right)\left(\frac{i \omega}{\omega_{c}}-e^{i \frac{5 \pi}{4}}\right)
$$

ou ainda:

$$
\mathrm{P}_{2}(\omega)=-\left(\frac{\omega}{\omega_{c}}\right)^{2}-\frac{i \omega}{\omega_{c}}\left(\cos \left(\frac{3 \pi}{4}\right)+\mathrm{i} \operatorname{sen}\left(\frac{3 \pi}{4}\right)+\cos \left(\frac{5 \pi}{4}\right)+\mathrm{i} \operatorname{sen}\left(\frac{5 \pi}{4}\right)\right)+1
$$

resultando-se finalmente: 


$$
P_{2}(\omega)=-\left(\frac{\omega}{\omega_{c}}\right)^{2}+i \sqrt{2} \frac{\omega}{\omega_{c}}+1
$$

cuja função de resposta em freqüência se expressa:

$$
\mathrm{H}_{2}(\omega)=\frac{1}{\mathrm{P}_{2}(\omega)}=\frac{1}{-\left(\frac{\omega}{\omega_{c}}\right)^{2}+\mathrm{i} \sqrt{2} \frac{\omega}{\omega_{c}}+1}
$$

cujo módulo vale:

$$
\left|H_{2}(\omega)\right|=\frac{1}{\left|P_{2}(\omega)\right|}=\frac{1}{\sqrt{\left(-\left(\frac{\omega}{\omega_{c}}\right)^{2}+1\right)^{2}+\left(\sqrt{2} \frac{\omega}{\omega_{c}}\right)^{2}}}
$$

resultando-se, pois:

$$
\left|H_{2}(\omega)\right|=\frac{1}{\sqrt{1+\left(\frac{\omega}{\omega_{c}}\right)^{4}}}
$$

como já esperado (vide eq. (2.34)).

Assim, de acordo com o expresso em (4.52), os coeficientes da correspondente equação diferencial ficam:

$$
\mathrm{a}_{2}=\left(\frac{1}{\omega_{\mathrm{c}}}\right)^{2} \quad ; \quad \mathrm{a}_{1}=\frac{\sqrt{2}}{\omega_{\mathrm{c}}} \quad ; \quad \mathrm{a}_{0}=1
$$

e, com isso, a equação diferencial apresenta a seguinte redação:

$$
\left(\frac{1}{\omega_{c}}\right)^{2} \frac{d^{2} y(t)}{{d t^{2}}^{2}}+\frac{\sqrt{2}}{\omega_{c}} \frac{d y(t)}{d t}+y(t)=x(t)
$$

como já discutido no Capítulo II.

No caso de três pólos, o polinômio $\mathrm{P}_{3}(\omega)$ é então redigido:

$$
P_{3}(\omega)=\left(\frac{i \omega}{\omega_{c}}-e^{i \frac{4 \pi}{6}}\right)\left(\frac{i \omega}{\omega_{c}}-e^{i \frac{6 \pi}{6}}\right)\left(\frac{i \omega}{\omega_{c}}-e^{i \frac{8 \pi}{6}}\right)
$$


que, uma vez expandido, fornece:

$$
P_{3}(\omega)=-i\left(\frac{\omega}{\omega_{c}}\right)^{3}-2\left(\frac{\omega}{\omega_{c}}\right)^{2}+2 i\left(\frac{\omega}{\omega_{c}}\right)+1
$$

A correspondente função de resposta em freqüência é dada pois na forma:

$$
H_{3}(\omega)=\frac{1}{P_{3}(\omega)}=\frac{1}{a_{3}(i \omega)^{3}+a_{2}(i \omega)^{2}+a_{1}(i \omega)+a_{0}}
$$

com os seguintes coeficientes:

$$
\mathrm{a}_{0}=1 \quad ; \quad \mathrm{a}_{1}=\frac{2}{\omega_{\mathrm{c}}} \quad ; \quad \mathrm{a}_{2}=\frac{2}{\omega_{\mathrm{c}}{ }^{2}} \quad ; \quad \mathrm{a}_{3}=\frac{1}{\omega_{\mathrm{c}}{ }^{3}}
$$

e cuja equação diferencial correspondente se escreve:

$$
\frac{1}{\omega_{c}^{3}} \frac{d^{3} y(t)}{{d t^{3}}^{3}}+\frac{2}{\omega_{c}^{2}} \frac{d^{2} y(t)}{{d t^{2}}^{2}}+\frac{2}{\omega_{c}} \frac{d y(t)}{d t}+y(t)=0
$$

encerrando-se assim esse caso.

No caso de quatro pólos o polinômio correspondente $\mathrm{P}_{4}(\omega)$ assume a forma:

$$
P_{4}(\omega)=\left(\frac{i \omega}{\omega_{c}}-e^{i \frac{5 \pi}{8}}\right)\left(\frac{i \omega}{\omega_{c}}-e^{i \frac{7 \pi}{8}}\right)\left(\frac{i \omega}{\omega_{c}}-e^{i \frac{9 \pi}{8}}\right)\left(\frac{i \omega}{\omega_{c}}-e^{i \frac{11 \pi}{8}}\right)
$$

sendo que sua expansão fornece:

$$
\mathrm{P}_{4}(\omega)=\left(\frac{\omega}{\omega_{c}}\right)^{4}-2,61313 \mathrm{i}\left(\frac{\omega}{\omega_{c}}\right)^{3}-3,41421\left(\frac{\omega}{\omega_{c}}\right)^{2}+2,61313 \mathrm{i}\left(\frac{\omega}{\omega_{c}}\right)+1
$$

o que corresponde à função de resposta em freqüência:

$$
\mathrm{H}_{4}(\omega)=\frac{1}{\mathrm{P}_{4}(\omega)}=\frac{1}{\mathrm{a}_{4} \omega^{4}-\mathrm{ia}_{3} \omega^{3}-\mathrm{a}_{2} \omega^{2}+\mathrm{ia}_{1} \omega+\mathrm{a}_{0}}
$$

com os seguintes coeficientes: 


$$
\begin{gathered}
\mathrm{a}_{0}=1 \quad ; \quad \mathrm{a}_{1}=2,61313\left(\frac{1}{\omega_{\mathrm{c}}}\right) ; \quad \mathrm{a}_{2}=3,41421\left(\frac{1}{\omega_{\mathrm{c}}}\right)^{2} \\
\mathrm{a}_{3}=2,61313\left(\frac{1}{\omega_{\mathrm{c}}}\right)^{3} \quad ; \quad \mathrm{a}_{4}=\left(\frac{1}{\omega_{\mathrm{c}}}\right)^{4}
\end{gathered}
$$

Concluindo-se, é oportuno apresentar em forma de tabela os coeficientes para os filtros de Butterworth com até 8 pólos, ressaltando-se que, embora o polinômio $P(\omega)$ seja formalmente igual ao polinômio de Butterworth com mudança de variável, no caso $B\left(s=i \omega / \omega_{c}\right)$, sua obtenção se deu de forma paralela, a partir da transformada de Fourier.

\begin{tabular}{|c|c|c|c|c|c|c|c|c|c|}
\hline $\begin{array}{c}\text { Número } \\
\text { de pólos }\end{array}$ & $\mathrm{a} 1$ & $\mathrm{a} 2$ & $\mathrm{a} 3$ & $\mathrm{a} 4$ & $\mathrm{a} 5$ & $\mathrm{a} 6$ & $\mathrm{a} 7$ & $\mathrm{a} 8$ & $\mathrm{a} 9$ \\
\hline 1 & 1 & 1 & 0 & 0 & 0 & 0 & 0 & 0 & 0 \\
\hline 2 & 1 & 1,414 & 1 & 0 & 0 & 0 & 0 & 0 & 0 \\
\hline 3 & 1 & 2 & 2 & 1 & 0 & 0 & 0 & 0 & 0 \\
\hline 4 & 1 & 2,613 & 3,414 & 2,613 & 1 & 0 & 0 & 0 & 0 \\
\hline 5 & 1 & 3,236 & 5,236 & 3,236 & 3,236 & 1 & 0 & 0 & 0 \\
\hline 6 & 1 & 3,864 & 7,464 & 9,141 & 7,464 & 3,864 & 1 & 0 & 0 \\
\hline 7 & 1 & 4,494 & 10,103 & 14,606 & 14,606 & 10,103 & 4,494 & 1 & 0 \\
\hline 8 & 1 & 5,126 & 13,138 & 21,848 & 25,691 & 21,848 & 13,138 & 5,126 & 1 \\
\hline
\end{tabular}

Tabela 4.1 - Coeficientes de Butterworth para até 8 pólos 


\section{Capítulo V}

\section{FILTRO DIGITAL COM QUADRATURA NUMÉRICA DE SEGUNDA ORDEM (TRAPEZOIDAL)}

\subsection{INTRODUÇÃO}

Uma vez mostrado no capítulo precedente que o processo de filtragem em freqüência segundo o modelo de Butterworth corresponde a uma integração de equação diferencial correspondente, cabe agora no presente capítulo desenvolver o clássico algoritmo de integração de segunda ordem de convergência, denominado algoritmo trapezoidal. Em outras palavras, o objetivo deste capítulo é o desenvolvimento de uma resolução numérica da equação diferencial de filtragem com uma entrada e um corte definido como $\omega_{\mathrm{c}}$. Nesse sentido são discutidas duas formulações, quais sejam, a do clássico método de Newmark[2] e uma outra mais conveniente que decorre do chamado método da redução matricial [5].

$\mathrm{Na}$ filtragem digital, os dados de entrada são, em geral, os captados pelo aparelho de mensuração, armazenando-se os valores da grandeza de interesse a cada intervalo de tempo especificado $\Delta$ t. Assim, o vetor de entrada é do tipo $x_{k}=x(k \Delta t)$, ou seja, sendo $x_{k} \circ k$-ésimo valor captado. A equação diferencial deve então ser representada por um algoritmo numérico equivalente, cuja integração resulta num vetor de resposta $\mathrm{y}_{\mathrm{k}}=\mathrm{y}(\mathrm{k} \Delta \mathrm{t})$.

A integração usual adotada nos textos clássicos da engenharia elétrica é, como já mencionado, a trapezoidal; e efetuada pela via da chamada transformação 
bilinear. Esta integração tem um erro de segunda ordem, ou seja, erro proporcional a $\Delta \mathrm{t}^{2}$ (quadrado do intervalo de tempo de captação).

Procura-se, de modo conveniente, desenvolver a forma geral do algoritmo para um número fixo de pólos, tendo-se em vista que o objetivo principal é a análise e a comparação de diferentes maneiras de resolução do problema. Por ser a equação mais trabalhada na engenharia de estruturas, será escolhida primeiramente a equação diferencial de segunda ordem. De início, faz-se uma analogia simples da equação de filtragem de dois pólos com a equação de movimento de um grau de liberdade.

\subsection{ANALOGIA COM A EQUAÇÃO DE MOVIMENTO}

Discutida anteriormente, a equação utilizada na dinâmica estrutural tem a conhecida forma:

$$
\ddot{\rho}(\mathrm{t})+2 \gamma \omega_{n} \dot{\rho}(t)+\omega_{n}^{2} \rho(t)=\omega_{n}^{2} f(t)
$$

e o filtro de dois pólos tem como equação diferencial:

$$
\ddot{\rho}(\mathrm{t})+\sqrt{2} \omega_{c} \dot{\rho}(\mathrm{t})+\omega_{\mathrm{c}}^{2} \rho(\mathrm{t})=\omega_{\mathrm{c}}^{2} \mathrm{f}(\mathrm{t})
$$

Assim, igualando-se os coeficientes de (5.1) e (5.2), chega-se a:

$$
\begin{aligned}
& \omega_{\mathrm{n}}=\omega_{\mathrm{c}} \\
& \gamma=\frac{\sqrt{2}}{2}
\end{aligned}
$$

implicando-se que o filtro de Butterworth de dois pólos corresponde ao caso de uma equação de movimento com freqüência de corte igual à freqüência natural e com amortecimento valendo $\sqrt{2} / 2$.

À titulo de registro, na engenharia elétrica, a equação diferencial de segunda ordem utilizada na resolução dos chamados circuitos LRC tem a forma análoga:

$$
L \ddot{q}(t)+R \dot{q}(t)+C q(t)=U(t)
$$


onde $\mathrm{L}$ é a indutância o circuito, $\mathrm{R}$ a resistência elétrica e $\mathrm{C}$ a capacitância. Valendo ainda registrar nesse ponto que os filtros de $\mathrm{N}$ pólos podem ser formados pela associação de vários filtros de dois pólos. Tal fato tem como fundamento a técnica conhecida como frações parciais, que reduz uma função (no caso $\mathrm{H}_{\mathrm{n}}(\mathrm{s})$ ) de denominador com grau polinomial maior, em várias parcelas de menor grau polinomial. Todavia tal propriedade é impraticável no caso estrutural, pois não é possível, por exemplo, calibrar o amortecimento do sistema, enquanto que a calibragem da resistência elétrica em um circuito é algo bastante simples.

\subsection{MÉTODO NEWMARK DE PASSO DUPLO}

Este método é muito comum na análise dinâmica em estruturas no caso de abordagem no domínio do tempo. Basicamente, o método Newmark provém de uma aproximação da derivada de uma função $\rho(t)$, dita trapezoidal, tendo-se como variáveis dependentes incógnitas com valores da função resposta $\rho_{\mathrm{i}}$ e sua derivada $\rho_{\mathrm{i}}^{\prime}$. Tais incógnitas são bastante apropriadas, pois trabalhar com grandezas em um único ponto no domínio do tempo é mais conveniente, dispensando-se a discretização da derivada, e tornando-a uma incógnita de iteração.

$\mathrm{Na}$ aproximação adotada no método Newmark assume-se que a aceleração é constante no intervalo entre dois pontos no domínio do tempo, fazendo com que a aceleração em um ponto $\xi$ pertencente ao intervalo $\Delta$ t seja considerada constante e avaliada como:

$$
\ddot{\rho}_{\xi}=\frac{1}{2}\left(\ddot{\rho}_{\mathrm{i}}+\ddot{\rho}_{\mathrm{i}+1}\right)
$$

ou seja, a semi-soma dos valores da aceleração nas extremidades do intervalo.

Integrando uma primeira vez (5.5) no tempo, variando-se de $t_{i}$ a $t_{i+1}$, que consistem, respectivamente, no início e no fim do intervalo $\Delta t$, tem-se:

$$
\dot{\rho}_{\mathrm{i}+1}=\dot{\rho}_{\mathrm{i}}+\Delta \mathrm{t} \frac{1}{2}\left(\ddot{\rho}_{\mathrm{i}}+\ddot{\rho}_{\mathrm{i}+1}\right)
$$


e, integrando pela segunda vez, resulta:

$$
\rho_{\mathrm{i}+1}=\rho_{\mathrm{i}}+\Delta \mathrm{t} \dot{\rho}_{\mathrm{i}}+\Delta \mathrm{t}^{2} \frac{1}{4}\left(\ddot{\rho}_{\mathrm{i}}+\ddot{\rho}_{\mathrm{i}+1}\right)
$$

ou ainda, isolando-se as parcelas contendo derivada segunda em (5.7) nos pontos i e i+1:

$$
\begin{aligned}
& \Delta \mathrm{t}^{2} \frac{1}{4}\left(\ddot{\rho}_{\mathrm{i}}+\ddot{\rho}_{\mathrm{i}+1}\right)=\rho_{\mathrm{i}+1}-\rho_{\mathrm{i}}-\Delta \mathrm{t} \dot{\rho}_{\mathrm{i}} \\
& \Delta \mathrm{t}^{2} \frac{1}{4}\left(\ddot{\rho}_{\mathrm{i}-1}+\ddot{\rho}_{\mathrm{i}}\right)=\rho_{\mathrm{i}}-\rho_{\mathrm{i}-1}-\Delta \mathrm{t} \dot{\rho}_{\mathrm{i}-1}
\end{aligned}
$$

Substituindo-se agora (5.8) em (5.6) tem-se finalmente:

$$
\begin{aligned}
& \Delta \mathrm{t} \frac{1}{2}\left(\dot{\rho}_{\mathrm{i}+1}+\dot{\rho}_{\mathrm{i}}\right)=\rho_{\mathrm{i}+1}-\rho_{\mathrm{i}} \\
& \Delta \mathrm{t} \frac{1}{2}\left(\dot{\rho}_{\mathrm{i}}+\dot{\rho}_{\mathrm{i}-1}\right)=\rho_{\mathrm{i}}-\rho_{\mathrm{i}-1}
\end{aligned}
$$

relações estas que vão ser usadas mais adiante.

As equações diferenciais para um filtro de dois pólos nos pontos i+1, i e i-1 são:

$$
\begin{aligned}
& \ddot{\rho}_{i+1}+\sqrt{2} \dot{\rho}_{\mathrm{i}+1}+\rho_{\mathrm{i}+1}=\mathrm{f}_{\mathrm{i}+1} \\
& \ddot{\rho}_{\mathrm{i}}+\sqrt{2} \dot{\rho}_{\mathrm{i}}+\rho_{\mathrm{i}}=\mathrm{f}_{\mathrm{i}} \\
& \ddot{\rho}_{\mathrm{i}-1}+\sqrt{2} \dot{\rho}_{\mathrm{i}-1}+\rho_{\mathrm{i}-1}=\mathrm{f}_{\mathrm{i}-1}
\end{aligned}
$$

Por outro lado, multiplicando-se a primeira e a terceira de (5.10) por $\Delta \mathrm{t}^{2} / 4$ e a segunda de (5.10) por $\Delta \mathrm{t}^{2} / 2$, e somando-se as três igualdades, tem-se:

$$
\Delta \mathrm{t}^{2}\left(\frac{\ddot{\rho}_{i+1}+\ddot{\rho}_{\mathrm{i}}}{4}+\frac{\ddot{\rho}_{\mathrm{i}-1}+\ddot{\rho}_{\mathrm{i}}}{4}\right)+\frac{\Delta \mathrm{t}^{2}}{2} \sqrt{2}\left(\frac{\dot{\rho}_{\mathrm{i}+1}+\dot{\rho}_{\mathrm{i}}}{2}+\frac{\dot{\rho}_{\mathrm{i}}+\dot{\rho}_{\mathrm{i}-1}}{2}\right)+\frac{\Delta \mathrm{t}^{2}}{4}\left(\rho_{\mathrm{i}+1}+2 \rho_{\mathrm{i}}+\rho_{\mathrm{i}-1}\right)=\Delta \mathrm{t}^{2}\left(\frac{\mathrm{f}_{\mathrm{i}+1}+\mathrm{f}_{\mathrm{i}-1}}{4}+\frac{\mathrm{f}_{\mathrm{i}}}{2}\right)
$$

cuja substituição de (5.8) e (5.9) na equação acima implica em: 


$$
\left[\rho_{\mathrm{i}+1}-\rho_{\mathrm{i}-1}-2 \rho_{\mathrm{i}}\right]+\frac{\Delta \mathrm{t}}{2} \sqrt{2}\left[\rho_{\mathrm{i}+1}-\rho_{\mathrm{i}-1}\right]+\frac{\Delta \mathrm{t}^{2}}{4}\left(\rho_{\mathrm{i}+1}+2 \rho_{\mathrm{i}}+\rho_{\mathrm{i}-1}\right)=\Delta \mathrm{t}^{2}\left(\frac{\mathrm{f}_{\mathrm{i}+1}+\mathrm{f}_{\mathrm{i}-1}}{4}+\frac{\mathrm{f}_{\mathrm{i}}}{2}\right)
$$

Isolando-se o termo $\rho_{i+1}$, chega-se finalmente em:

$$
\left(1+\frac{\Delta t}{2} \sqrt{2}+\frac{\Delta t^{2}}{4}\right) \rho_{i+1}=\left(2-\frac{\Delta t^{2}}{2}\right) \rho_{i}-\left(1-\frac{\Delta t}{2} \sqrt{2}+\frac{\Delta t^{2}}{4}\right) \rho_{i-1}+\Delta t^{2}\left(\frac{f_{i+1}+f_{i-1}}{4}+\frac{f_{i}}{2}\right)
$$

que corresponde à conhecida forma direta do método Newmark. Salienta-se que tal expressão coincide com a obtida através da transformada $Z$, utilizada pelos engenheiros elétricos.

\subsection{MÉTODO DE NEWMARK DE PASSO SIMPLES}

Retomando-se a clássica equação de movimento na sua forma adimensional (filtragem de Butterworth de dois pólos), ou seja:

$$
\ddot{\rho}(\mathrm{t})+2 \gamma \omega_{n} \dot{\rho}(\mathrm{t})+\omega_{\mathrm{n}}^{2} \rho(\mathrm{t})=\omega_{\mathrm{n}}^{2} \mathrm{f}(\mathrm{t})
$$

o procedimento para o emprego do método Newmark na sua formulação de passo simples decorre da integração trapezoidal, sendo admitido:

$$
\begin{aligned}
& \dot{\rho}_{i+1}=\dot{\rho}_{i}+\frac{\Delta t}{2}\left(\ddot{\rho}_{i}+\ddot{\rho}_{i+1}\right) \\
& \rho_{i+1}=\rho_{i}+\Delta t \dot{\rho}_{i}+\frac{\Delta t^{2}}{4}\left(\ddot{\rho}_{i}+\ddot{\rho}_{i+1}\right)
\end{aligned}
$$

como exposto em (5.6) e em (5.7). Aplicando-se agora a equação (5.14) nos pontos i e i+1 e somando-se membro a membro os termos, ou seja:

$$
\begin{gathered}
\ddot{\rho}_{\mathrm{i}+1}+2 \gamma \omega_{\mathrm{n}} \dot{\rho}_{\mathrm{i}+1}+\omega_{\mathrm{n}}^{2} \rho_{\mathrm{i}+1}=\omega_{\mathrm{n}}^{2} \mathrm{f}_{\mathrm{i}+1} \\
+ \\
\ddot{\rho}_{\mathrm{i}}+2 \gamma \omega_{\mathrm{n}} \dot{\rho}_{\mathrm{i}}+\omega_{\mathrm{n}}^{2} \rho_{\mathrm{i}}=\omega_{\mathrm{n}}^{2} \mathrm{f}_{\mathrm{i}} \\
= \\
\left(\ddot{\rho}_{\mathrm{i}+1}+\ddot{\rho}_{\mathrm{i}}\right)+2 \gamma \omega_{\mathrm{n}}\left(\dot{\rho}_{\mathrm{i}+1}+\dot{\rho}_{\mathrm{i}}\right)+\omega_{\mathrm{n}}^{2}\left(\rho_{\mathrm{i}+1}+\rho_{\mathrm{i}}\right)=\omega_{\mathrm{n}}^{2}\left(\mathrm{f}_{\mathrm{i}+1}+\mathrm{f}_{\mathrm{i}}\right)
\end{gathered}
$$

verifica-se que a substituição da primeira de (5.15) na última de (5.16) resulta: 


$$
\frac{1}{\Delta t}\left(\dot{\rho}_{i+1}-\dot{\rho}_{i}\right)+\gamma \omega_{n}\left(\dot{\rho}_{i+1}+\dot{\rho}_{i}\right)+\frac{\omega_{n}^{2}}{2}\left(\rho_{i+1}+\rho_{i}\right)=\frac{\omega_{n}^{2}}{2}\left(f_{i+1}+f_{i}\right)
$$

ou ainda, multiplicando ambos os membros de (5.17) por $\Delta \mathrm{t}^{2}$ :

$$
\Delta t\left(\dot{\rho}_{i+1}-\dot{\rho}_{i}\right)+\gamma \omega_{n} \Delta t\left(\dot{\rho}_{i+1}+\dot{\rho}_{i}\right) \Delta t+\frac{\omega_{n}^{2} \Delta t^{2}}{2}\left(\rho_{i+1}+\rho_{i}\right)=\frac{\omega_{n}^{2} \Delta t^{2}}{2}\left(f_{i+1}+f_{i}\right)
$$

Sendo mais cômoda a notação:

$$
\Delta \mathrm{t}\left(\dot{\rho}_{\mathrm{i}+1}-\dot{\rho}_{\mathrm{i}}\right)+\gamma \theta\left(\dot{\rho}_{\mathrm{i}+1}+\dot{\rho}_{\mathrm{i}}\right) \Delta \mathrm{t}+\frac{\theta^{2}}{2}\left(\rho_{\mathrm{i}+1}+\rho_{\mathrm{i}}\right)=\frac{\theta^{2}}{2}\left(\mathrm{f}_{\mathrm{i}+1}+\mathrm{f}_{\mathrm{i}}\right)
$$

onde

$$
\theta=\omega_{n} \Delta t
$$

ou ainda, em notação vetorial:

$$
\left\{\begin{array}{cc}
\frac{\theta^{2}}{2} & 1+\gamma \theta
\end{array}\right\}\left\{\begin{array}{c}
\rho_{\mathrm{i}+1} \\
\Delta \mathrm{t} \dot{\rho}_{\mathrm{i}+1}
\end{array}\right\}=\left\{\begin{array}{cc}
\frac{-\theta^{2}}{2} & 1-\gamma \theta
\end{array}\right\}\left\{\begin{array}{c}
\rho_{\mathrm{i}} \\
\Delta \mathrm{t} \dot{\rho}_{\mathrm{i}}
\end{array}\right\}+\left\{\begin{array}{cc}
\frac{\theta^{2}}{2} & \frac{\theta^{2}}{2}
\end{array}\right\}\left\{\begin{array}{c}
\mathrm{f}_{\mathrm{i}} \\
\mathrm{f}_{\mathrm{i}+1}
\end{array}\right\}
$$

correspondendo à primeira equação do sistema. A segunda equação provém da substituição da segunda de (5.15) na última de (5.16), ou seja:

$$
\frac{4}{\Delta \mathrm{t}^{2}}\left(\rho_{\mathrm{i}+1}-\rho_{\mathrm{i}}-\Delta \mathrm{t} \dot{\rho}_{\mathrm{i}}\right)+2 \gamma \omega_{\mathrm{n}}\left(\dot{\rho}_{\mathrm{i}+1}+\dot{\rho}_{\mathrm{i}}\right)+\omega_{\mathrm{n}}^{2}\left(\rho_{\mathrm{i}+1}+\rho_{\mathrm{i}}\right)=\omega_{\mathrm{n}}^{2}\left(\mathrm{f}_{\mathrm{i}+1}+\mathrm{f}_{\mathrm{i}}\right)
$$

onde multiplicando ambos os lados de (5.22) por $\Delta \mathrm{t}^{2} / 4$ e aplicando o expresso em (5.20), tem-se:

$$
\left(\rho_{i+1}-\rho_{\mathrm{i}}-\Delta \mathrm{t} \dot{\rho}_{\mathrm{i}}\right)+\frac{\gamma \theta}{2}\left(\dot{\rho}_{\mathrm{i}+1}+\dot{\rho}_{\mathrm{i}}\right) \Delta \mathrm{t}+\frac{\theta^{2}}{4}\left(\rho_{\mathrm{i}+1}+\rho_{\mathrm{i}}\right)=\frac{\theta^{2}}{4}\left(\mathrm{f}_{\mathrm{i}+1}+\mathrm{f}_{\mathrm{i}}\right)
$$

que em forma vetorial se redige:

$$
\left\{1+\frac{\theta^{2}}{4} \frac{\gamma \theta}{2}\right\}\left\{\begin{array}{c}
\rho_{\mathrm{i}+1} \\
\Delta \mathrm{t} \dot{\rho}_{\mathrm{i}+1}
\end{array}\right\}=\left\{1-\frac{\theta^{2}}{4} \quad 1-\frac{\gamma \theta}{2}\right\}\left\{\begin{array}{c}
\rho_{\mathrm{i}} \\
\Delta \mathrm{t} \dot{\rho}_{\mathrm{i}}
\end{array}\right\}+\left\{\begin{array}{cc}
\frac{\theta^{2}}{4} & \frac{\theta^{2}}{4}
\end{array}\right\}\left\{\begin{array}{c}
\mathrm{f}_{\mathrm{i}} \\
\mathrm{f}_{\mathrm{i}+1}
\end{array}\right\}
$$

representando-se a segunda equação referida. Assim sendo, reunindo-se em notação matricial o expresso em (5.21) e (5.24) tem-se: 


$$
\left[\begin{array}{cc}
\frac{\theta^{2}}{2} & 1+\gamma \theta \\
1+\frac{\theta^{2}}{4} & \frac{\gamma \theta}{2}
\end{array}\right]\left\{\begin{array}{c}
\rho_{\mathrm{i}+1} \\
\Delta t \dot{\rho}_{\mathrm{i}+1}
\end{array}\right\}=\left[\begin{array}{cc}
\frac{-\theta^{2}}{2} & 1-\gamma \theta \\
1-\frac{\theta^{2}}{4} & 1-\frac{\gamma \theta}{2}
\end{array}\right]\left\{\begin{array}{c}
\rho_{\mathrm{i}} \\
\Delta \mathrm{t} \dot{\rho}_{\mathrm{i}}
\end{array}\right\}+\left[\begin{array}{cc}
\frac{\theta^{2}}{2} & \frac{\theta^{2}}{2} \\
\frac{\theta^{2}}{4} & \frac{\theta^{2}}{4}
\end{array}\right]\left\{\begin{array}{c}
\mathrm{f}_{\mathrm{i}} \\
\mathrm{f}_{\mathrm{i}+1}
\end{array}\right\}
$$

que, no caso do filtro de pólo duplo, substituindo-se o valor de $\gamma$ por $\sqrt{2} / 2$ temse:

$$
\left[\begin{array}{cc}
\frac{\theta^{2}}{2} & 1+\frac{\sqrt{2}}{2} \theta \\
1+\frac{\theta^{2}}{4} & \frac{\sqrt{2} \theta}{4}
\end{array}\right]\left\{\begin{array}{c}
\mathrm{y}_{\mathrm{i}+1} \\
\Delta \dot{y}_{\mathrm{i}+1}
\end{array}\right\}=\left[\begin{array}{cc}
\frac{-\theta^{2}}{2} & 1-\frac{\sqrt{2}}{2} \theta \\
1-\frac{\theta^{2}}{4} & 1-\frac{\sqrt{2} \theta}{4}
\end{array}\right]\left\{\begin{array}{c}
\mathrm{y}_{\mathrm{i}} \\
\Delta \dot{t y}_{\mathrm{i}}
\end{array}\right\}+\left[\begin{array}{cc}
\frac{\theta^{2}}{2} & \frac{\theta^{2}}{2} \\
\frac{\theta^{2}}{4} & \frac{\theta^{2}}{4}
\end{array}\right]\left\{\begin{array}{c}
\mathrm{x}_{\mathrm{i}} \\
\mathrm{x}_{\mathrm{i}+1}
\end{array}\right\}
$$

encerrando-se assim a formulação da versão digital do filtro em questão na forma iterativa (integração direta), ou passo a passo.

\subsection{EXEMPLOS DE APLICAÇÃO}

No sentido de exemplificar a aplicação e aferir a eficiência do algoritmo apresentado, considere-se o caso da filtragem de três diferentes sinais de entrada. $O$ filtro terá nestes casos uma freqüência de corte $\omega_{\mathrm{c}}=50 \mathrm{rad} / \mathrm{s}$, e com um passo $\Delta \mathbf{t}=\mathbf{0 , 0 5 s}$. A entrada analisada inicialmente é uma senóide simples do tipo $\mathbf{x}(\mathbf{t})=\mathbf{s e n}(\mathbf{2 0 t})$. Nesse caso, tem-se:

$$
\theta=\omega_{c} \Delta t=50 \times 0,05=2,5 \mathrm{rad}
$$

conduzindo a um algoritmo do tipo:

$$
\left[\begin{array}{cc}
3,125 & 2,268 \\
2,563 & 0,884
\end{array}\right]\left\{\begin{array}{c}
y_{i+1} \\
\Delta t \dot{y}_{i+1}
\end{array}\right\}=\left[\begin{array}{cc}
-3,125 & -0,768 \\
-0,563 & 0,116
\end{array}\right]\left\{\begin{array}{c}
y_{i} \\
\Delta t \dot{y}_{i}
\end{array}\right\}+\left[\begin{array}{cc}
3,125 & 3,125 \\
1,563 & 1,563
\end{array}\right]\left\{\begin{array}{c}
x_{i} \\
x_{i+1}
\end{array}\right\}
$$

O sinal $y_{i}$ está mostrado em forma de gráfico na figura 5.1. Percebese que a amplitude da resposta é praticamente a do sinal de entrada com uma mínima redução. Isto ocorre porque, para este valor adotado de freqüência de corte, tem-se que $\left|\mathrm{H}_{2}(20)\right|=0,987$. 


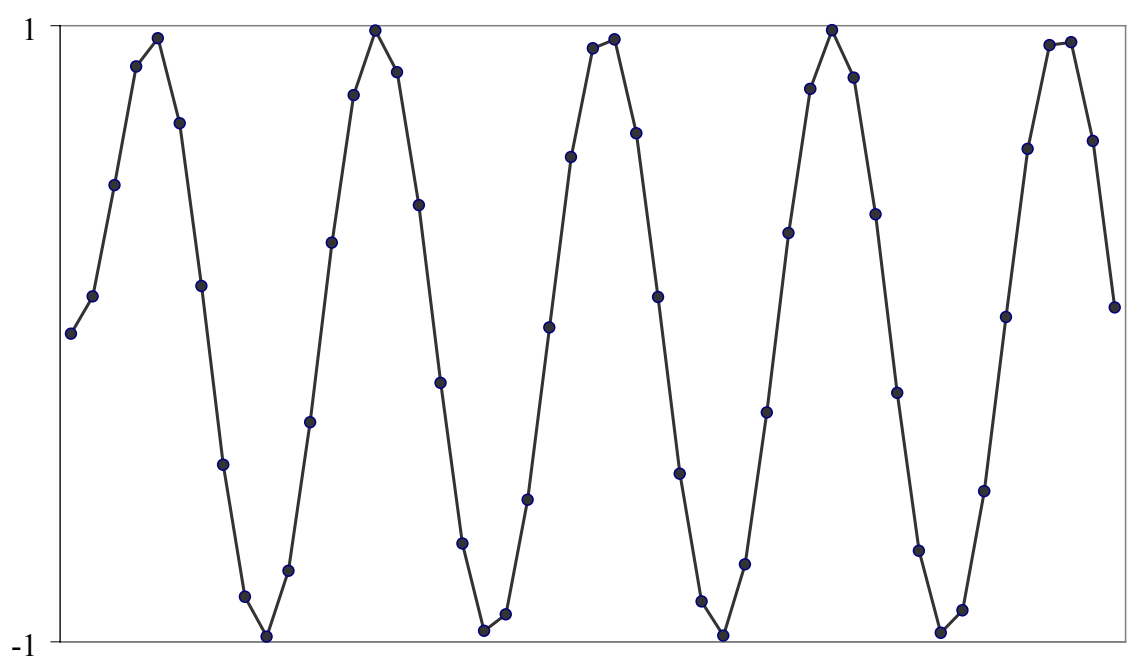

Fig. 5.1 - Saída do algoritmo de Newmark para baixa freqüência

Verificando a eficiência para uma entrada de freqüência maior, por exemplo, $\mathbf{x}(\mathbf{t})=\operatorname{sen}(\mathbf{7 0 t})$, com $\Delta \mathbf{t}=\mathbf{0 , 0 2 s}$, nota-se no resultado lançado na figura 5.2 , que a resposta em amplitude já é menor.

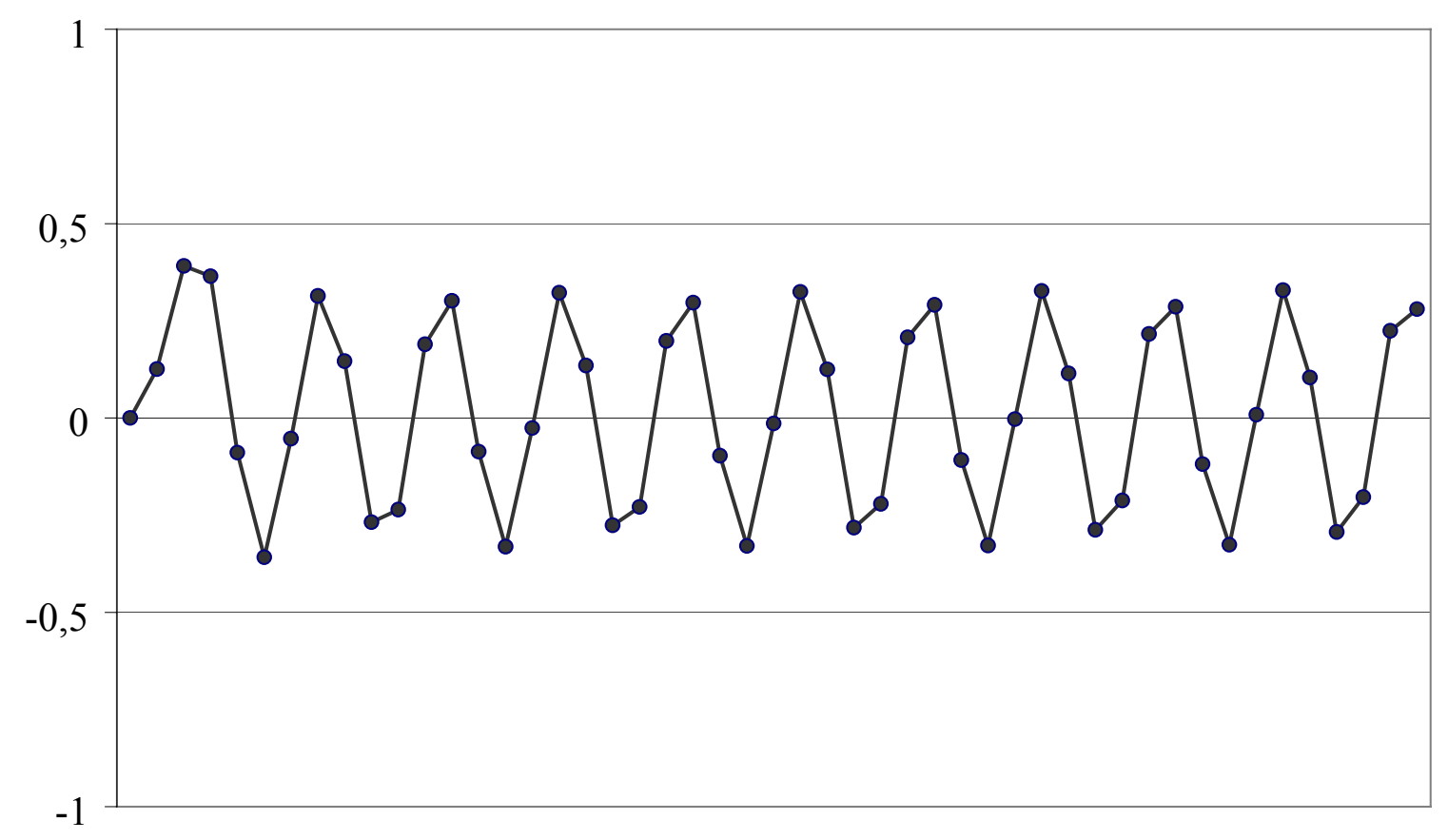

Fig. 5.2 - Saída do algoritmo de Newmark para alta freqüência

Finalmente, considerando uma função de entrada na forma $x(t)=\operatorname{sen}(20 t)+0,2 \operatorname{sen}(100 t)$, com $\Delta t=0,02 s$, pode-se perceber qual é a eficiência real da filtragem. A figura 5.3 mostra o sinal de saída obtido pelo método. 


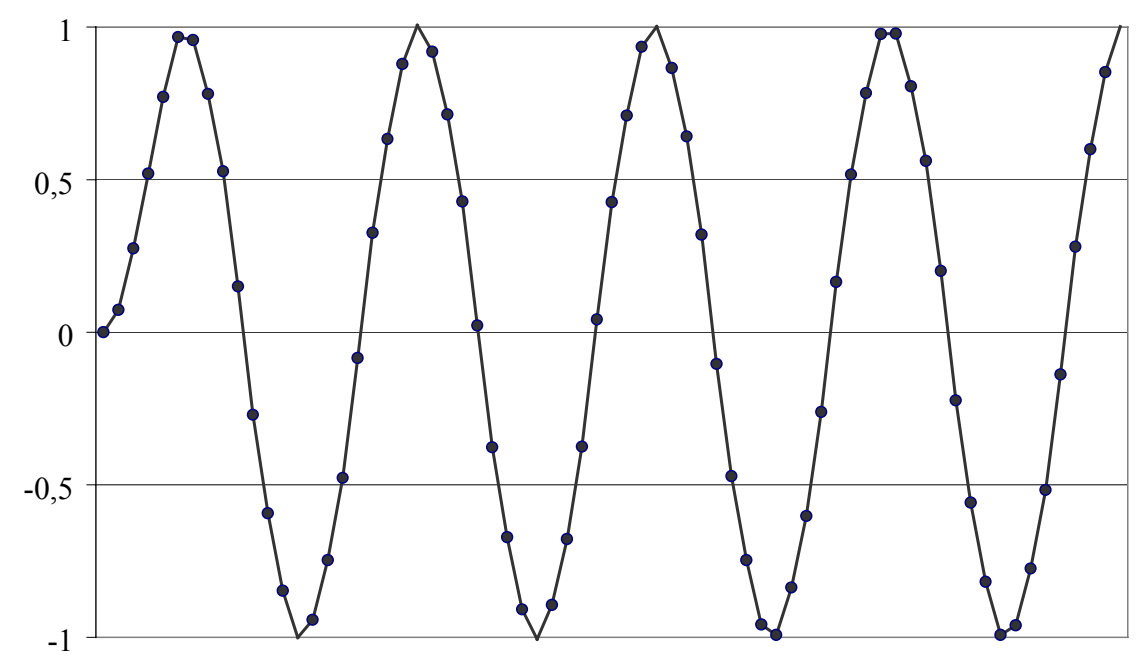

Fig. 5.3 - Saída do algoritmo de Newmark para entrada contendo alta e baixa freqüência

Vale assinalar que outros exemplos vão ser objeto de estudo mais detalhado no capítulo especialmente dedicado ao tema.

\subsection{FORMULAÇÃO MATRICIAL REDUZIDA DA EQUAÇÃO DIFERENCIAL}

No sentido de facilitar a geração dos algoritmos de filtragem para um número genérico de pólos, é conveniente o recurso à uma formulação de fácil entendimento, uniformizando o procedimento via redução direta da ordem da equação diferencial para uma de primeira ordem. Assim, o emprego do operador de primeira ordem pode ser estendido para a solução da filtragem com número genérico de pólos.

Para tanto, considere-se uma equação diferencial genérica de ordem $\mathrm{N}$ como mostrado no que se segue:

$$
a_{N} \frac{d^{N} y(t)}{d t^{N}}+a_{N-1} \frac{d^{N-1} y(t)}{d t^{N-1}}+\ldots+a_{1} \frac{d y(t)}{d t}+a_{0} y(t)=b_{0} x(t)
$$

É fácil verificar que a seguinte forma matricial pode ser redigida: 


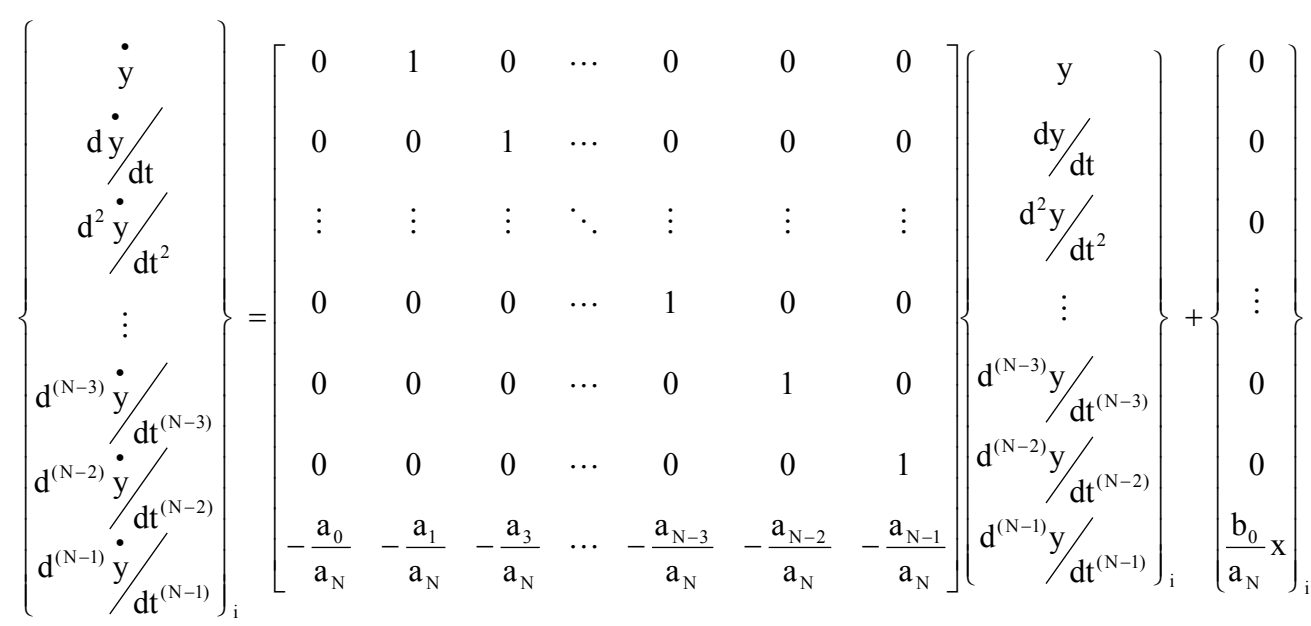

onde se tem nas $\mathrm{N}-1$ primeiras linhas identidades elementares e na última a própria equação diferencial em questão. A equação matricial (5.30) representa em verdade um sistema de equações diferencias de primeira ordem, ou seja:

$$
\{\dot{\mathrm{Y}}\}_{\mathrm{i}}=[\mathrm{A}]\{\mathrm{Y}\}_{\mathrm{i}}+\{\mathrm{X}\}_{\mathrm{i}}
$$

e com isso facultando-se a aplicação de operadores mais elementares na sua integração.

A integração trapezoidal aplicada na equação (5.31) inicia-se com a relação vetorial equivalente à primeira das (5.9), ou seja:

$$
\{\mathrm{Y}\}_{\mathrm{i}+1}=\{\mathrm{Y}\}_{\mathrm{i}}+\frac{\Delta \mathrm{t}}{2}\left(\{\dot{\mathrm{Y}}\}_{\mathrm{i}}+\{\dot{\mathrm{Y}}\}_{\mathrm{i}+1}\right)
$$

Multiplicando-se agora a igualdade (5.31) por $\Delta \mathrm{t} / 2$ nos pontos i e i+1, e somando-se membro a membro, ou seja:

$$
\begin{gathered}
\frac{\Delta \mathrm{t}}{2}\left[\{\dot{\mathrm{Y}}\}_{\mathrm{i}}=\{\mathrm{X}\}_{\mathrm{i}}+[\mathrm{A}]\{\mathrm{Y}\}_{\mathrm{i}}\right] \\
+ \\
\frac{\Delta \mathrm{t}}{2}\left[\{\dot{\mathrm{Y}}\}_{\mathrm{i}+1}=\{\mathrm{X}\}_{\mathrm{i}+1}+[\mathrm{A}]\{\mathrm{Y}\}_{\mathrm{i}+1}\right]
\end{gathered}
$$

e tendo-se em conta (5.32), a equação matricial de recorrência torna-se então:

$$
\left([\mathrm{I}]-\frac{\Delta \mathrm{t}}{2}[\mathrm{~A}]\right)\{\mathrm{Y}\}_{\mathrm{i}+1}=\frac{\Delta \mathrm{t}}{2}\left[\{\mathrm{X}\}_{\mathrm{i}}+\{\mathrm{X}\}_{\mathrm{i}+1}\right]+\left([\mathrm{I}]+\frac{\Delta \mathrm{t}}{2}[\mathrm{~A}]\right)\{\mathrm{Y}\}_{\mathrm{i}}
$$


ou ainda, denominando-se:

$$
\begin{gathered}
{[\mathrm{G}]=[\mathrm{I}]-\frac{\Delta \mathrm{t}}{2}[\mathrm{~A}]} \\
{\left[\mathrm{G}^{*}\right]=[\mathrm{I}]+\frac{\Delta \mathrm{t}}{2}[\mathrm{~A}]} \\
\{\mathrm{F}\}_{\mathrm{i}}=\frac{\Delta \mathrm{t}}{2}\left[\{\mathrm{X}\}_{\mathrm{i}}+\{\mathrm{X}\}_{\mathrm{i}+1}\right]
\end{gathered}
$$

pode-se de maneira genérica explicitar a equação (5.34) na forma:

$$
[\mathrm{G}]\{\mathrm{Y}\}_{\mathrm{i}+1}=\left[\mathrm{G}^{*}\right]\{\mathrm{Y}\}_{\mathrm{i}}+\{\mathrm{F}\}_{\mathrm{i}}
$$

tendo-se com isso, um algoritmo de solução do problema, uma vez que, como as condições iniciais no problema da filtragem são nulas (são nulas a função e suas derivadas até ordem $\mathrm{N}-1$, no instante inicial).

\subsection{APLICAÇÃO PARA FILTRO DE DOIS PÓLOS}

No caso particular de dois pólos, os coeficientes da equação diferencial fazem com que as matrizes $[A],[G]$ e $\left[G^{*}\right]$ adquiram as formas:

$$
\begin{aligned}
& {[\mathrm{A}]=\left[\begin{array}{cc}
0 & 1 \\
-\omega_{\mathrm{c}}^{2} & -\omega_{\mathrm{c}} \sqrt{2}
\end{array}\right]} \\
& {[\mathrm{G}]=\left[\begin{array}{cc}
1 & -\frac{\Delta \mathrm{t}}{2} \\
\frac{\Delta \mathrm{t}}{2} \omega_{\mathrm{c}}^{2} & 1+\frac{\Delta \mathrm{t}}{2} \omega_{\mathrm{c}} \sqrt{2}
\end{array}\right]} \\
& {\left[\mathrm{G}^{*}\right]=\left[\begin{array}{cc}
1 & \frac{\Delta \mathrm{t}}{2} \\
-\frac{\Delta \mathrm{t}}{2} \omega_{\mathrm{c}}^{2} & 1-\frac{\Delta \mathrm{t}}{2} \omega_{\mathrm{c}} \sqrt{2}
\end{array}\right]}
\end{aligned}
$$

implicando-se que a iteração matricial a ser executada venha a ser:

$$
\left[\begin{array}{cc}
1 & -\frac{\Delta t}{2} \\
\frac{\Delta t}{2} \omega_{c}^{2} & 1+\frac{\Delta t}{2} \omega_{c} \sqrt{2}
\end{array}\right]\left\{\begin{array}{l}
y \\
\dot{y}
\end{array}\right\}_{i+1}=\left[\begin{array}{cc}
1 & \frac{\Delta t}{2} \\
-\frac{\Delta t}{2} \omega_{c}^{2} & 1-\frac{\Delta t}{2} \omega_{c} \sqrt{2}
\end{array}\right]\left\{\begin{array}{l}
y \\
\dot{y}
\end{array}\right\}_{i}+\frac{\Delta t}{2}\left(\left\{\begin{array}{l}
0 \\
x
\end{array}\right\}_{i}+\left\{\begin{array}{l}
0 \\
x
\end{array}\right\}_{i+1}\right)
$$


A título de exemplo, retomando-se aquele caso de filtragem com freqüência de corte $\omega_{c}=50 \mathrm{rad} / \mathrm{s}$, passo $\Delta t=0,03 \mathrm{~s}$ e entrada $x(t)=\operatorname{sen}(20 t)$, a operação indicada em (5.38) fornece o resultado lançado no gráfico da figura 5.4; e no caso de uma entrada $\mathbf{x}(\mathbf{t})=\mathbf{s e n}(\mathbf{7 0 t})$ ), com $\Delta t=0,02 s$, o resultado encontra-se lançado no gráfico da figura 5.5 .

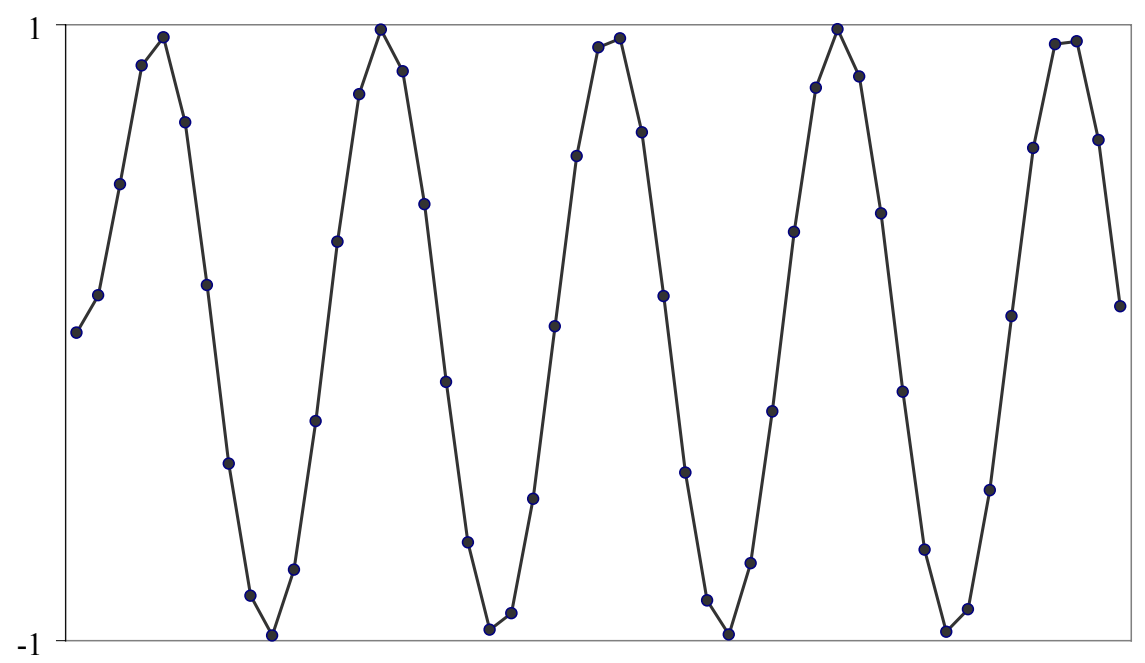

Fig. 5.4 - Saída do algoritmo trapezoidal para baixa freqüência

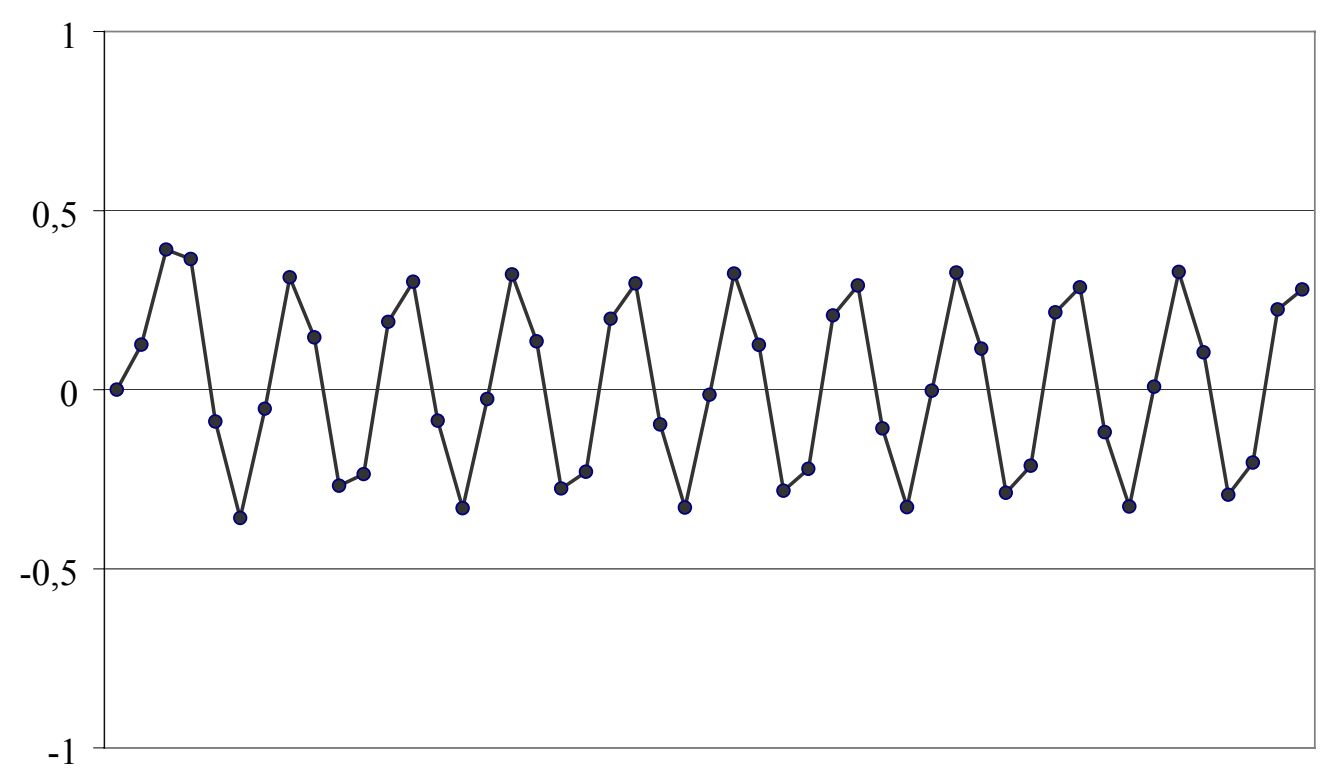

Fig. 5.5 - Saída do algoritmo trapezoidal para alta freqüência 


\subsection{FILTRAGEM UTILIZANDO NÚMERO DE PÓLOS MAIS ELEVADO}

A facilidade de implementação do algoritmo de integração trapezoidal através da forma matricial reduzida permite desenvolver os correspondentes filtros com maior número de pólos. Em FORTRAN, desenvolveu-se um programa que contém algoritmos de filtragem de até quinze pólos. Isso permite realizar uma filtragem mais eficiente do ponto de vista da conservação do sinal desejado e eliminação do sinal indesejado.

As figuras 5.6, 5.7 e 5.8 exibem os resultados da filtragem de um sinal de entrada contendo duas senóides superpostas, ou seja:

$$
x(t)=\operatorname{sen}(30 t)+0,3 \operatorname{sen}(70 t)
$$

com a utilização de um algoritmo trapezoidal contemplando cinco, dez e quinze pólos respectivamente, e sendo considerada uma freqüência de corte $\omega_{c}=50 \mathrm{rad} / \mathrm{s}$, e discretização com passo $\Delta \mathbf{t}=\mathbf{0 , 0 1 s}$. Em linha pontilhada encontra-se o sinal de entrada e em linha contínua o sinal de saída. Notando-se que, conforme aumentase o número de pólos, melhora-se o resultado desejado, que deve resultar na componente de menor freqüência em (5.39). Por outro lado, o aumento do número de pólos acarreta um atraso na estabilização harmônica do sinal de saída, como esperado. Assim sendo, dependendo da duração do sinal, utiliza-se um filtro com número de pólos que permita atingir a estabilidade harmônica em tempo desejado. De forma simplificada, este atraso na resposta do sistema pode ser explicado devido ao fato de que todas as condições iniciais são nulas, tornando-se a equação diferencial de ordem $\mathrm{N}$ com valores nulos no instante zero da função e de suas derivadas até a ordem $\mathrm{N}-1$. A forma matemática de se explicar este atraso consiste no fato de $\mathrm{P}(\omega)$ ser formado por um produto de números complexos, sendo que o módulo de $\mathrm{P}(\omega)$ resulta do produto dos módulos de cada complexo, e o argumento de $\mathrm{P}(\omega)$ é a soma dos argumentos desses mesmos números complexos. 
53

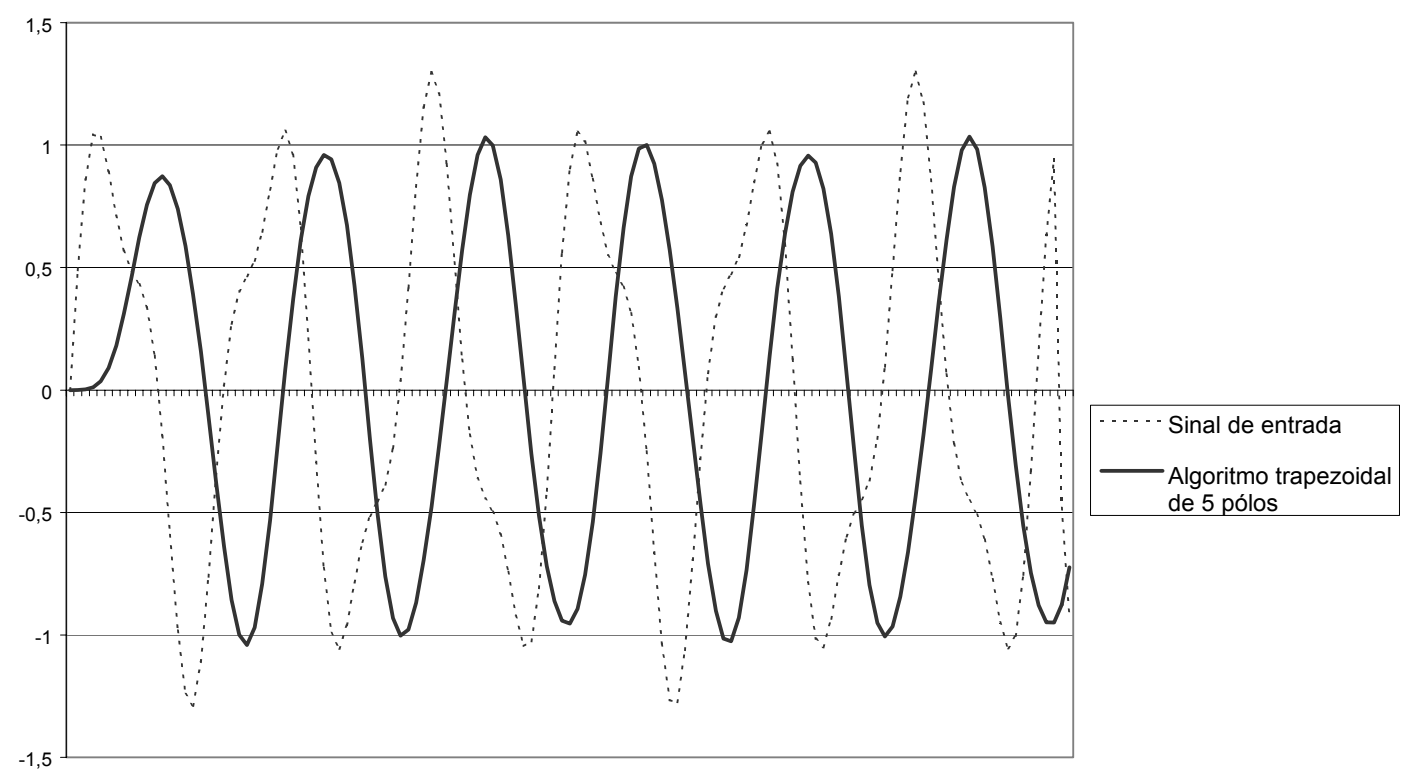

Figura 5.6 - Análise do filtro trapezoidal de cinco pólos

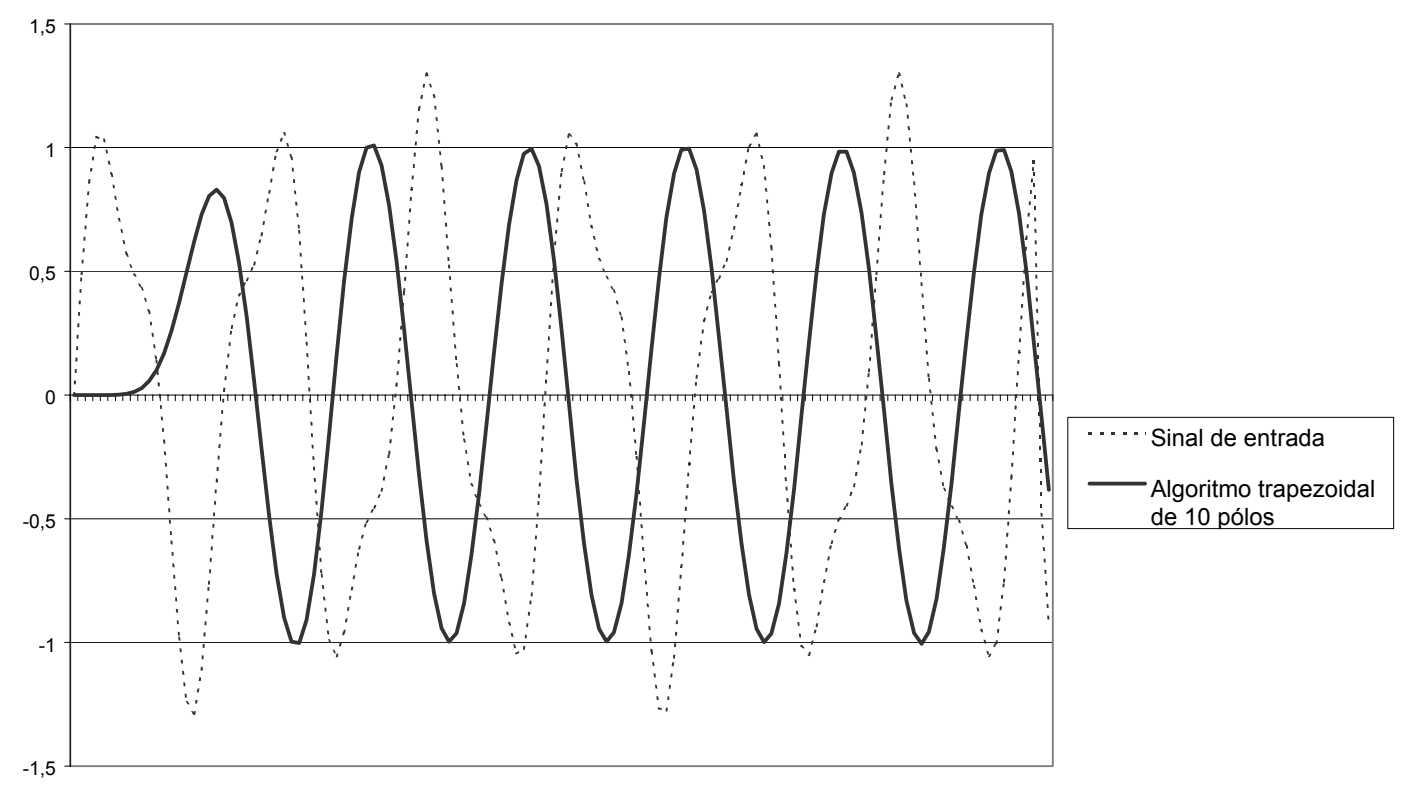

Figura 5.7 - Análise do filtro trapezoidal de dez pólos 


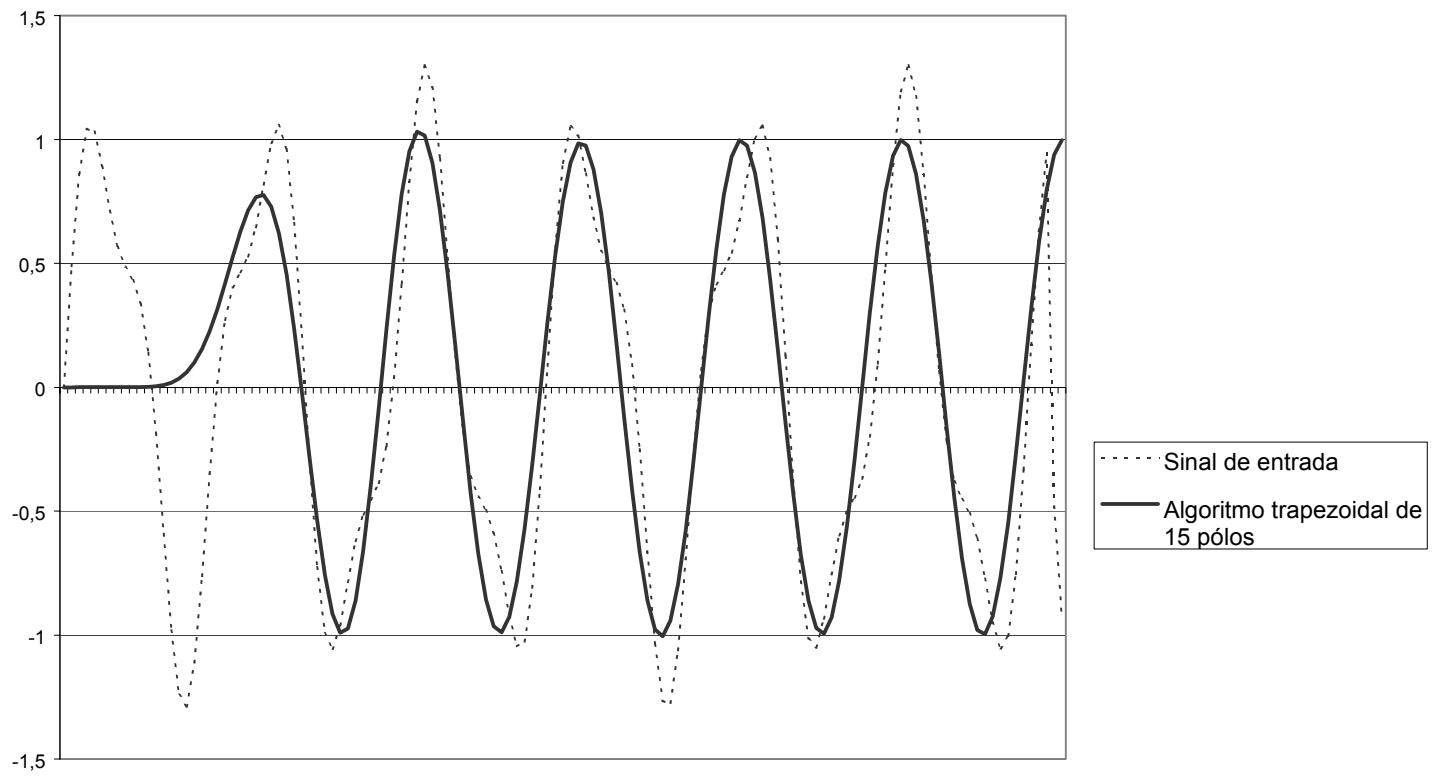

Figura 5.8 - Análise do filtro trapezoidal de quinze pólos

De maneira geral, o resultado obtido no operador trapezoidal é satisfatório, mostrando-se estável e de fácil programação em FORTRAN. 


\section{Capítulo VI}

\section{FILTRO DIGITAL COM QUADRATURA NUMÉRICA DE ORDEM SUPERIOR (OPERADOR HERMITIANO)}

\subsection{INTRODUÇÃO}

Neste capítulo é estudado um operador Hermitiano [6] que permite formular um algoritmo de integração direta de equações diferenciais com ordem de erro local do quinto grau. Tal operador provém de uma operação de quadratura numérica na qual se considera também a segunda derivada da função sendo integrada. Trata-se de um algoritmo que expande uma combinação da função e derivadas em dois pontos sucessivos i e i+1 (algoritmo de passo único), de sorte a anular as parcelas de ordem de erro mais baixo, mediante a consideração das séries de Taylor correspondentes.

É oportuno assinalar que o integrador trapezoidal descrito no capítulo precedente (dado pela equação (5.32)) é, em verdade, um operador hermitiano que combina valores da função e sua primeira derivada nos pontos i e i+1, anulando-se as parcelas de primeira e segunda ordem.

A exemplo do realizado no capítulo precedente, o foco inicial é a equação diferencial de segunda ordem (equação de movimento), para a qual se consegue formular um algoritmo de integração passo a passo, seguido de exemplos de aplicação tendo por finalidade examinar o seu funcionamento. Porém, visto que o algoritmo passa a exigir a consideração da primeira derivada da função de entrada, torna-se necessário também providenciar um operador que para expressar tal derivada com a ordem de erro compatível. 
Integradores com maior grau de precisão podem ser obtidos mediante a consideração de uma combinação hermitiana contendo maior grau de derivação, conforme extensão apresentada também neste capítulo.

A formulação geral para o caso com qualquer número de pólos é alcançada com base numa extensão da formulação matricial (5.31), já bem discutida anteriormente. Essa formulação geral, embora seja de maior complexidade, pode ser obtida percorrendo-se os mesmos caminhos traçados pelo capítulo $\mathrm{V}$, sendo que a única mudança de monta decorre do surgimento de um grau a mais na derivada do vetor de resposta.

\subsection{OPERADOR HERMITIANO COM DERIVADA DE SEGUNDA ORDEM}

O chamado operador hermitiano consiste numa combinação de valores da função de interesse e de suas derivadas nos pontos sucessivos i e i+1 da variável independente, de tal sorte que sejam anuladas as parcelas com ordem de erro mais baixo na combinação. Exemplificando-se, seja considerada então a seguinte combinação:

$$
\mathrm{c}_{1} \mathrm{y}_{\mathrm{i}}+\mathrm{c}_{2} \mathrm{y}_{\mathrm{i}+1}+\mathrm{c}_{3} \Delta \mathrm{t} \dot{\mathrm{y}}_{\mathrm{i}}+\mathrm{c}_{4} \Delta \mathrm{t} \dot{\mathrm{y}}_{\mathrm{i}+1}+\mathrm{c}_{5} \Delta \mathrm{t}^{2} \ddot{\mathrm{y}}_{\mathrm{i}}+\mathrm{c}_{6} \Delta \mathrm{t}^{2} \ddot{\mathrm{y}}_{\mathrm{i}+1}
$$

para a qual se procura um certo grau de precisão. Nesse sentido, examinandose a expansão da função y e de suas derivadas, a avaliação de $y_{i+1}$ e suas derivadas nesse ponto seguinte por meio das respectivas séries de Taylor podem assim se expressar:

$$
\begin{aligned}
& \mathrm{y}_{\mathrm{i}+1}=\mathrm{y}_{\mathrm{i}}+\left.\Delta \mathrm{t} \frac{\mathrm{dy}}{\mathrm{dt}}\right|_{i}+\left.\frac{\Delta \mathrm{t}^{2}}{2} \frac{\mathrm{d}^{2} \mathrm{y}}{\mathrm{dt}^{2}}\right|_{\mathrm{i}}+\left.\frac{\Delta \mathrm{t}^{3}}{6} \frac{\mathrm{d}^{3} \mathrm{y}}{\mathrm{dt}^{3}}\right|_{\mathrm{i}}+\cdots=\left.\sum_{\mathrm{k}=0}^{\infty} \frac{\Delta \mathrm{t}^{\mathrm{k}}}{\mathrm{k} !} \frac{\mathrm{d}^{\mathrm{k}} \mathrm{y}}{\mathrm{dt}^{\mathrm{k}}}\right|_{\mathrm{i}} \\
& \left.\frac{\mathrm{dy}}{\mathrm{dt}}\right|_{\mathrm{i}+1}=\left.\frac{\mathrm{dy}}{\mathrm{dt}}\right|_{i}+\left.\Delta t \frac{\mathrm{d}^{2} \mathrm{y}}{\mathrm{dt}^{2}}\right|_{i}+\left.\frac{\Delta \mathrm{t}^{2}}{2} \frac{\mathrm{d}^{3} \mathrm{y}}{\mathrm{dt}^{3}}\right|_{i}+\left.\frac{\Delta \mathrm{t}^{3}}{6} \frac{\mathrm{d}^{4} \mathrm{y}}{\mathrm{dt}^{4}}\right|_{i}+\cdots=\left.\sum_{\mathrm{k}=0}^{\infty} \frac{\Delta \mathrm{t}^{\mathrm{k}}}{\mathrm{k} !} \frac{\mathrm{d}^{(\mathrm{k}+1)} \mathrm{y}}{\mathrm{dt}^{(\mathrm{k}+1)}}\right|_{\mathrm{i}} \\
& \left.\frac{\mathrm{d}^{2} \mathrm{y}}{\mathrm{dt}^{2}}\right|_{\mathrm{i}+1}=\left.\frac{\mathrm{d}^{2} \mathrm{y}}{\mathrm{dt}^{2}}\right|_{i}+\left.\Delta \mathrm{t} \frac{\mathrm{d}^{3} \mathrm{y}}{\mathrm{dt}^{3}}\right|_{i}+\left.\frac{\Delta \mathrm{t}^{2}}{2} \frac{\mathrm{d}^{4} \mathrm{y}}{\mathrm{dt}^{4}}\right|_{i}+\left.\frac{\Delta \mathrm{t}^{3}}{6} \frac{\mathrm{d}^{5} \mathrm{y}}{\mathrm{dt}^{5}}\right|_{i}+\cdots=\left.\sum_{\mathrm{k}=0}^{\infty} \frac{\Delta \mathrm{t}^{\mathrm{k}}}{\mathrm{k} !} \frac{\mathrm{d}^{(\mathrm{k}+2)} \mathrm{y}}{\mathrm{dt}^{(\mathrm{k}+2)}}\right|_{i}
\end{aligned}
$$

Tendo-se em conta agora as igualdades (6.2), o expresso em (6.1) ganha a seguinte redação: 


$$
\mathrm{c}_{1} \mathrm{y}_{\mathrm{i}}+\left.\mathrm{c}_{2} \sum_{\mathrm{k}=0}^{\infty} \frac{\Delta \mathrm{t}^{\mathrm{k}}}{\mathrm{k} !} \frac{\mathrm{d}^{\mathrm{k}} \mathrm{y}}{\mathrm{dt} \mathrm{t}^{\mathrm{k}}}\right|_{\mathrm{i}}+\left.\mathrm{c}_{3} \Delta \mathrm{t} \frac{\mathrm{dy}}{\mathrm{dt}}\right|_{\mathrm{i}}+\left.\mathrm{c}_{4} \Delta \mathrm{t} \sum_{\mathrm{k}=0}^{\infty} \frac{\Delta \mathrm{t}^{\mathrm{k}}}{\mathrm{k} !} \frac{\mathrm{d}^{(\mathrm{k}+1)} \mathrm{y}}{\mathrm{dt}^{(\mathrm{k}+1)}}\right|_{\mathrm{i}}+\left.\mathrm{c}_{5} \Delta \mathrm{t}^{2} \frac{\mathrm{d}^{2} \mathrm{y}}{\mathrm{dt}^{2}}\right|_{\mathrm{i}}+\left.\mathrm{c}_{6} \Delta \mathrm{t}^{2} \sum_{\mathrm{k}=0}^{\infty} \frac{\Delta \mathrm{t}^{\mathrm{k}}}{\mathrm{k} !} \frac{\mathrm{d}^{(\mathrm{k}+2)} \mathrm{y}}{\mathrm{dt}^{(\mathrm{k}+2)}}\right|_{\mathrm{i}}
$$

ou ainda, fatorando-se as derivadas de $\mathrm{y}_{\mathrm{i}}$ :

$$
\begin{aligned}
& \left(\mathrm{c}_{1}+\mathrm{c}_{2}\right) \mathrm{y}_{\mathrm{i}}+\left.\left(\mathrm{c}_{2}+\mathrm{c}_{3}+\mathrm{c}_{4}\right) \Delta \mathrm{t} \frac{\mathrm{dy}}{\mathrm{dt}}\right|_{\mathrm{i}}+\left.\left(\frac{\mathrm{c}_{2}}{2}+\mathrm{c}_{4}+\mathrm{c}_{5}+\mathrm{c}_{6}\right) \Delta \mathrm{t}^{2} \frac{\mathrm{d}^{2} \mathrm{y}}{\mathrm{dt}^{2}}\right|_{\mathrm{i}}+\left.\left(\frac{\mathrm{c}_{2}}{6}+\frac{\mathrm{c}_{4}}{2}+\mathrm{c}_{6}\right) \Delta \mathrm{t}^{3} \frac{\mathrm{d}^{3} \mathrm{y}}{\mathrm{dt}^{3}}\right|_{\mathrm{i}}+\ldots \\
& \ldots+\left.\left(\frac{\mathrm{c}_{2}}{24}+\frac{\mathrm{c}_{4}}{6}+\frac{\mathrm{c}_{6}}{2}\right) \Delta \mathrm{t}^{4} \frac{\mathrm{d}^{4} \mathrm{y}}{\mathrm{dt}^{4}}\right|_{\mathrm{i}}+\left.\left(\frac{\mathrm{c}_{2}}{120}+\frac{\mathrm{c}_{4}}{24}+\frac{\mathrm{c}_{6}}{6}\right) \Delta \mathrm{t}^{5} \frac{\mathrm{d}^{5} \mathrm{y}}{\mathrm{dt}^{5}}\right|_{\mathrm{i}}+\left.\left(\frac{\mathrm{c}_{2}}{720}+\frac{\mathrm{c}_{4}}{120}+\frac{\mathrm{c}_{6}}{24}\right) \Delta \mathrm{t}^{6} \frac{\mathrm{d}^{6} \mathrm{y}}{\mathrm{dt}^{6}}\right|_{\mathrm{i}}+\cdots
\end{aligned}
$$

cuja anulação, por exemplo, das parcelas com ordem de erro até quarta ordem, já na forma matricial implica em:

$$
\left[\begin{array}{cccccc}
1 & 0 & 0 & 0 & 0 & 0 \\
1 & 1 & 0 & 0 & 0 & 0 \\
0 & 1 & 1 & 1 & 0 & 0 \\
0 & 1 / 2 & 0 & 1 & 1 & 1 \\
0 & 1 / 6 & 0 & 1 / 2 & 0 & 1 \\
0 & 1 / 24 & 0 & 1 / 6 & 0 & 1 / 2
\end{array}\right]\left\{\begin{array}{l}
c_{1} \\
c_{2} \\
c_{3} \\
c_{4} \\
c_{5} \\
c_{6}
\end{array}\right\}=\left\{\begin{array}{l}
1 \\
0 \\
0 \\
0 \\
0 \\
0
\end{array}\right\}
$$

ou de forma abreviada:

$$
[\beta]\{c\}=\{f\}
$$

sendo que a primeira equação apenas assume valor unitário para a constante $\mathrm{c}_{1}$. A solução do sistema de equações (6.5) resulta:

$$
\begin{aligned}
& c_{1}=1 \\
& c_{2}=-1 \\
& c_{3}=1 / 2 \\
& c_{4}=1 / 2 \\
& c_{5}=1 / 12 \\
& c_{6}=-1 / 12
\end{aligned}
$$

Procurando-se trabalhar apenas com valores inteiros, convém multiplicar-se os coeficientes expressos em (6.7) por 12. Assim, o operador hermitiano em questão representa-se pela combinação: 


$$
12 \mathrm{y}_{\mathrm{i}}-12 \mathrm{y}_{\mathrm{i}+1}+6 \Delta \mathrm{t} \dot{\mathrm{y}}_{\mathrm{i}}+6 \Delta \mathrm{t} \dot{\mathrm{y}}_{\mathrm{i}+1}+\Delta \mathrm{t}^{2} \ddot{\mathrm{y}}_{\mathrm{i}}-\Delta \mathrm{t}^{2} \ddot{\mathrm{y}}_{\mathrm{i}+1}=0+\mathrm{O}\left(\Delta \mathrm{t}^{5}\right)
$$

cuja derivação e multiplicação por $\Delta t$ permite escrever-se:

$$
12 \Delta t \dot{y}_{i}-12 \Delta t \dot{y}_{i+1}+6 \Delta t^{2} \ddot{y}_{i}+6 \Delta t^{2} \ddot{y}_{i+1}+\Delta t^{3} \dddot{y}_{i}-\Delta t^{3} \dddot{y}_{i+1}=0+O\left(\Delta t^{6}\right)
$$

sendo o expresso por (6.8) e (6.9) conhecidos como operadores hermitianos de ordem 5 e 6 , respectivamente.

\subsection{CASO GERAL DE OPERADORES}

No sentido de se generalizar o tratamento dos operadores hermitianos para v;Arias ordens, seja considerada uma combinação da função y com derivada até uma dada ordem $M$. Tem-se assim $M+1$ incógnitas no ponto i e $M+1$ incógnitas no ponto $i+1$, totalizando-se $2 M+2$ incógnitas. Dessa forma a matriz $[\beta]$, conforme expressa em (6.6), passa a ter dimensão $(2 M+2) \times(2 M+2)$.

Admitindo-se que para as $2 \mathrm{M}+2$ equações em questão uma das incógnitas pode ser arbitrada, reserva-se a primeira linha de $[\beta]$ para esta escolha. É interessante notar que os últimos coeficientes apresentam magnitudes cada vez menores em valor, e, além disso, dois a dois os coeficientes ora são de mesmo sinal, ora de sinais contrários. Então, pela comodidade de se trabalhar com valores inteiros positivos, fixa-se o valor do penúltimo coeficiente como sendo unitário.

Contando agora com as $2 \mathrm{M}+1$ equações restantes, a k-gésima delas ocupa a linha $k+1$, e tem a contribuição de coeficientes pares como sendo:

$$
\mathrm{a}_{\mathrm{k}+1,2 \mathrm{j}}=\frac{1}{(\mathrm{k}-\mathrm{j}) !} \quad \text { para o domínio } \mathrm{k} \geq \mathrm{j}
$$

Por outro lado, os colunas ímpares de [ $\beta]$ tem termos unitários de forma escalonada a cada duas colunas e nas primeiras linhas somente, começandose da segunda linha em diante. Pode-se então generalizar a matriz [ $\beta]$ na forma: 


$$
[\beta]=\left[\begin{array}{ccccccccc}
0 & 0 & 0 & 0 & \cdots & 0 & 0 & 1 & 0 \\
1 & 1 /(1-1) ! & 0 & 0 & \cdots & 0 & 0 & 0 & 0 \\
0 & 1 /(2-1) ! & 1 & 1 /(1-1) ! & \cdots & 0 & 0 & 0 & 0 \\
0 & 1 /(3-1) ! & 0 & 1 /(2-1) ! & \cdots & 0 & 0 & 0 & 0 \\
\vdots & \vdots & \vdots & \vdots & \ddots & \vdots & \vdots & \vdots & \vdots \\
0 & 1 /(2 \mathrm{M}-3) ! & 0 & 1 /(2 \mathrm{M}-4) ! & \cdots & 0 & 1 /(\mathrm{M}-2) ! & 0 & 1 /(\mathrm{M}-3) ! \\
0 & 1 /(2 \mathrm{M}-2) ! & 0 & 1 /(2 \mathrm{M}-3) ! & \cdots & 0 & 1 /(\mathrm{M}-1) ! & 0 & 1 /(\mathrm{M}-2) ! \\
0 & 1 /(2 \mathrm{M}-1) ! & 0 & 1 /(2 \mathrm{M}-2) ! & \cdots & 0 & 1 / \mathrm{M} ! & 0 & 1 /(\mathrm{M}-1) ! \\
0 & 1 /(2 \mathrm{M}) ! & 0 & 1 /(2 \mathrm{M}-1) ! & \cdots & 0 & 1 /(\mathrm{M}+1) ! & 0 & 1 / \mathrm{M} !
\end{array}\right]
$$

Esta matriz, como facilmente se verifica, é de fácil programação em linguagem FORTRAN. Nota-se que para $M=2$, o resultado confere com (6.5).

\subsection{APLICAÇÃO NO ALGORITMO DE DOIS PÓLOS}

Seja retomada a equação diferencial de um filtro de Butterworth de dois pólos, ou seja:

$$
\ddot{y}(t)=\omega_{c}^{2} x(t)-\omega_{c} \sqrt{2} \dot{y}(t)-\omega_{c}^{2} y(t)
$$

Multiplicando-se (6.12) por $\Delta \mathrm{t}^{2}$, e chamando-se $\theta=\omega_{\mathrm{c}} \Delta \mathrm{t}$, de maneira similar ao já feito em no desenvolvimento do método de Newmark, tem-se:

$$
\Delta \mathrm{t}^{2} \ddot{\mathrm{y}}(\mathrm{t})=\theta^{2} \mathrm{x}(\mathrm{t})-\Delta \mathrm{t} \theta \sqrt{2} \dot{\mathrm{y}}(\mathrm{t})-\theta^{2} \mathrm{y}(\mathrm{t})
$$

Derivando-se e multiplicando-se ambos os membros de (6.13) por $\Delta$ t resulta:

$$
\Delta \mathrm{t}^{3} \dddot{\mathrm{y}}(\mathrm{t})=\Delta \mathrm{t} \theta^{2} \dot{\mathrm{x}}(\mathrm{t})-\Delta \mathrm{t}^{2} \theta \sqrt{2} \ddot{\mathrm{y}}(\mathrm{t})-\Delta \mathrm{t} \theta^{2} \dot{\mathrm{y}}(\mathrm{t})
$$

resultado esse que, face ao redigido em (6.13) e em (6.14), permite agora redigir:

$$
\Delta \mathrm{t}^{3} \dddot{\mathrm{y}}(\mathrm{t})=\Delta \mathrm{t} \theta^{2} \dot{\mathrm{x}}(\mathrm{t})-\theta^{3} \sqrt{2} \mathrm{x}(\mathrm{t})+\Delta \mathrm{t} \theta^{2} \dot{\mathrm{y}}(\mathrm{t})+\theta^{3} \sqrt{2} \mathrm{y}(\mathrm{t})
$$

Tendo-se em conta (6.13) e (6.15), a equação (6.8) e (6.9), agrupadas em notação matricial fornece: 


$$
\begin{aligned}
& \left(\begin{array}{cc}
6+\theta \sqrt{2} & \theta^{2}-12 \\
-\theta^{2}-6 \theta \sqrt{2}-12 & -\sqrt{2} \theta^{3}-6 \theta^{2}
\end{array}\right)\left\{\begin{array}{c}
\Delta t . \dot{y}_{i+1} \\
y_{i+1}
\end{array}\right\}=\left(\begin{array}{cc}
\theta \sqrt{2}-6 & \theta^{2}-12 \\
-\theta^{2}+6 \theta \sqrt{2}-12 & 6 \theta^{2}-\sqrt{2} \theta^{3}
\end{array}\right)\left\{\begin{array}{c}
\Delta t . \dot{y}_{i} \\
y_{1}
\end{array}\right\}+ \\
& \left(\begin{array}{cc}
0 & -\theta^{2} \\
-\theta^{2} & \sqrt{2} \theta^{3}-6 \theta^{2}
\end{array}\right)\left\{\begin{array}{c}
\Delta t . \dot{x}_{i} \\
x_{i}
\end{array}\right\}+\left(\begin{array}{cc}
0 & \theta^{2} \\
\theta^{2} & -\sqrt{2} \theta^{3}-6 \theta^{2}
\end{array}\right)\left\{\begin{array}{c}
\Delta t . \dot{x}_{i+1} \\
x_{1+1}
\end{array}\right\}
\end{aligned}
$$

cabendo notar haver agora o aparecimento dos termos de derivadas do sinal de entrada. Todavia, isso apenas implica na necessidade de se elaborar os algoritmos correspondentes para o sinal de entrada $\mathrm{x}$ na forma discretizada $\mathrm{e}$ com uma ordem de erro compatível com a dos operadores envolvidos. Para tanto, deve-se recorrer novamente ao correspondente desenvolvimento da série de Taylor com truncamento resultando uma ordem de erro compatível.

\subsection{DISCRETIZAÇÃO DA DERIVADA DO SINAL DE ENTRADA}

A combinação hermitiana a ser considerada deve apenas envolver os valores da função de entrada $x_{k}$, via de regra, os dados coletados pelo aparelho. A quantidade de valores necessários para conferir ordem sexta de erro local $\left(\Delta t^{6}\right)$, que é a ordem de erro da equação (6.9), é calculada fazendo com que o número de parcelas a serem anuladas na combinação hermitiana contemple até parcelas multiplicadas por $\Delta \mathrm{t}^{5} \mathrm{~d}^{5} \mathrm{x}(\mathrm{t}) / \mathrm{dt} \mathrm{t}^{5}$. Isto resulta em seis parcelas necessárias, implicando então no operador hermitiano:

$$
\Delta \mathrm{t} . \dot{\mathrm{x}}_{\mathrm{i}}=\mathrm{d}_{0} \mathrm{x}_{\mathrm{i}}+\mathrm{d}_{1} \mathrm{x}_{\mathrm{i}+1}+\mathrm{d}_{2} \mathrm{x}_{\mathrm{i}+2}+\mathrm{d}_{3} \mathrm{x}_{\mathrm{i}+3}+\mathrm{d}_{4} \mathrm{x}_{\mathrm{i}+4}+\mathrm{d}_{5} \mathrm{x}_{\mathrm{i}+5}
$$

sendo que as primeiras expansões de Taylor correspondentes são as seguintes:

$$
\begin{gathered}
x_{i}=x_{i} \\
x_{i+1}=x_{i}+\left.\Delta t \frac{d x}{d t}\right|_{i}+\left.\frac{\Delta t^{2}}{2} \frac{d^{2} x}{{d t^{2}}^{2}}\right|_{i}+\left.\frac{\Delta t^{3}}{6} \frac{d^{3} x}{d t^{3}}\right|_{i}+\left.\frac{\Delta t^{4}}{24} \frac{d^{4} x}{d t^{4}}\right|_{i}+\left.\frac{\Delta t^{5}}{120} \frac{d^{5} x}{d t^{5}}\right|_{i} \\
x_{i+2}=x_{i}+\left.2 \Delta t \frac{d x}{d t}\right|_{i}+\left.\frac{(2 \Delta t)^{2}}{2} \frac{d^{2} x}{d t^{2}}\right|_{i}+\left.\frac{(2 \Delta t)^{3}}{6} \frac{d^{3} x}{{d t^{3}}^{3}}\right|_{i}+\left.\frac{(2 \Delta t)^{4}}{24} \frac{d^{4} x}{d t^{4}}\right|_{i}+\left.\frac{(2 \Delta t)^{5}}{120} \frac{d^{5} x}{d^{5}}\right|_{i} \\
x_{i+3}=x_{i}+\left.3 \Delta t \frac{d x}{d t}\right|_{i}+\left.\frac{(3 \Delta t)^{2}}{2} \frac{d^{2} x}{d^{2}}\right|_{i}+\left.\frac{(3 \Delta t)^{3}}{6} \frac{d^{3} x}{d^{3}}\right|_{i}+\left.\frac{(3 \Delta t)^{4}}{24} \frac{d^{4} x}{d t^{4}}\right|_{i}+\left.\frac{(3 \Delta t)^{5}}{120} \frac{d^{5} x}{d t^{5}}\right|_{i}
\end{gathered}
$$


Assim, efetuando-se as necessárias substituições de (6.18) em (6.17) leva a:

$$
\begin{aligned}
& \left.\Delta \mathrm{t} \frac{\mathrm{dx}}{\mathrm{dt}}\right|_{\mathrm{i}}=\left(\mathrm{d}_{0}+\mathrm{d}_{1}+\mathrm{d}_{2}+\mathrm{d}_{3}+\mathrm{d}_{4}+\mathrm{d}_{5}\right) \mathrm{x}_{\mathrm{i}}+\left.\left(\mathrm{d}_{1}+2 \mathrm{~d}_{2}+3 \mathrm{~d}_{3}+4 \mathrm{~d}_{4}+5 \mathrm{~d}_{5}\right) \Delta \mathrm{t} \frac{\mathrm{dx}}{\mathrm{dt}}\right|_{\mathrm{i}}+ \\
& +\left.\left(\frac{\mathrm{d}_{1}+2^{2} \mathrm{~d}_{2}+3^{2} \mathrm{~d}_{3}+4^{2} \mathrm{~d}_{4}+5^{2} \mathrm{~d}_{5}}{2}\right) \Delta \mathrm{t}^{2} \frac{\mathrm{d}^{2} \mathrm{x}}{\mathrm{dt}^{2}}\right|_{\mathrm{i}}+\left.\left(\frac{\mathrm{d}_{1}+2^{3} \mathrm{~d}_{2}+3^{3} \mathrm{~d}_{3}+4^{3} \mathrm{~d}_{4}+5^{3} \mathrm{~d}_{5}}{6}\right) \Delta \mathrm{t}^{3} \frac{\mathrm{d}^{3} \mathrm{x}}{\mathrm{dt}^{3}}\right|_{\mathrm{i}}+ \\
& +\left.\left(\frac{\mathrm{d}_{1}+2^{4} \mathrm{~d}_{2}+3^{4} \mathrm{~d}_{3}+4^{4} \mathrm{~d}_{4}+5^{4} \mathrm{~d}_{5}}{24}\right) \Delta \mathrm{t}^{4} \frac{\mathrm{d}^{4} \mathrm{x}}{\mathrm{dt}^{4}}\right|_{\mathrm{i}}+\left.\left(\frac{\mathrm{d}_{1}+2^{5} \mathrm{~d}_{2}+3^{5} \mathrm{~d}_{3}+4^{5} \mathrm{~d}_{4}+5^{5} \mathrm{~d}_{5}}{120}\right) \Delta \mathrm{t}^{5} \frac{\mathrm{d}^{5} \mathrm{x}}{\mathrm{dt}^{5}}\right|_{\mathrm{i}}
\end{aligned}
$$

valendo-se ressaltar que, aparentemente, a generalização para um caso genérico de grau $\mathrm{N}$ na ordem de erro da primeira derivada, pode ser assim redigido:

$$
\left.\Delta \mathrm{t} \frac{\mathrm{dx}}{\mathrm{dt}}\right|_{\mathrm{i}}=\mathrm{d}_{0} \mathrm{x}_{\mathrm{i}}+\left(\sum_{\mathrm{k}=0}^{\mathrm{N}}\left(\sum_{\mathrm{m}=1}^{\mathrm{N}} \frac{\mathrm{d}_{\mathrm{m}} \mathrm{m}^{\mathrm{k}}}{\mathrm{k} !}\right) \Delta \mathrm{t}^{\mathrm{k}} \frac{\mathrm{d}^{\mathrm{k}} \mathrm{x}}{\left.\mathrm{dt^{ \textrm {k } }}\right|_{\mathrm{i}}}\right)
$$

ou mesmo para um grau $\mathrm{J}$ da derivada, necessário para integradores com maior precisão, de modo análogo:

$$
\left.\Delta \mathrm{t}^{\mathrm{J}} \frac{\mathrm{d}^{\mathrm{J}} \mathrm{x}}{\mathrm{dt}^{\mathrm{J}}}\right|_{i}=\mathrm{d}_{0} \mathrm{x}_{\mathrm{i}}+\left(\left.\sum_{\mathrm{k}=0}^{\mathrm{N}}\left(\sum_{\mathrm{m}=1}^{\mathrm{N}} \frac{\mathrm{d}_{\mathrm{m}} \mathrm{m}^{\mathrm{k}}}{\mathrm{k} !}\right) \Delta \mathrm{t}^{\mathrm{k}} \frac{\mathrm{d}^{\mathrm{k}} \mathrm{x}}{\mathrm{dt}^{\mathrm{k}}}\right|_{\mathrm{i}}\right)
$$

A anulação das parcelas presentes em (6.19) expressa em forma matricial assim se redige:

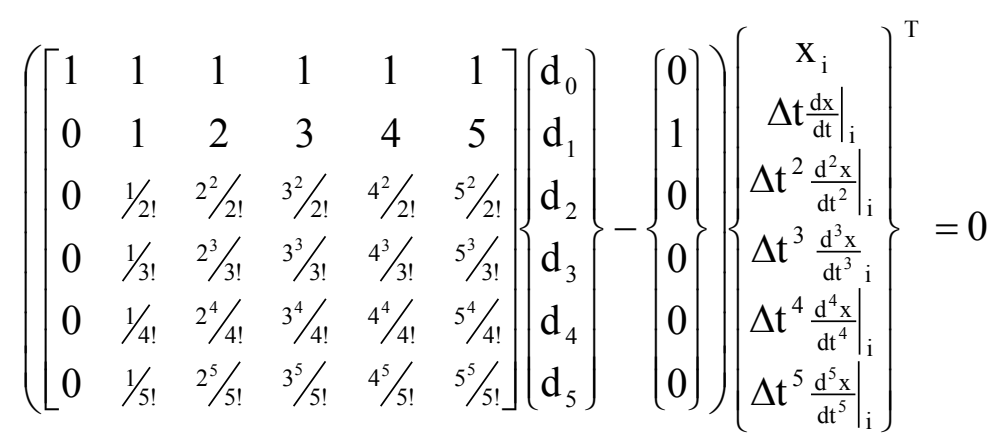

cuja solução não trivial implica em: 


$$
\left[\begin{array}{cccccc}
1 & 1 & 1 & 1 & 1 & 1 \\
0 & 1 & 2 & 3 & 4 & 5 \\
0 & 1 / 2 ! & 2^{2} / 2 ! & 3^{2} / 2 ! & 4^{2} / 2 ! & 5^{2} / 2 ! \\
0 & 1 / 3 ! & 2^{3} / 3 ! & 3^{3} / 3 ! & 4^{3} / 3 ! & 5^{3} / 3 ! \\
0 & 1 / 4 ! & 2^{3} / 4 ! & 3^{3} / 4 ! & 4^{4} / 4 ! & 5^{4} / 4 ! \\
0 & 1 / 5 ! & 2^{5} / 5 ! & 3^{5} / 5 ! & 4^{5} / 5 ! & 5^{5} / 5 !
\end{array}\right]\left\{\begin{array}{l}
\mathrm{d}_{0} \\
\mathrm{~d}_{1} \\
\mathrm{~d}_{2} \\
\mathrm{~d}_{3} \\
\mathrm{~d}_{4} \\
\mathrm{~d}_{5}
\end{array}\right\}=\left\{\begin{array}{l}
0 \\
1 \\
0 \\
0 \\
0 \\
0
\end{array}\right\}
$$

resultando pois:

$$
\begin{aligned}
& \mathrm{d}_{0}=-137 / 60 \\
& \mathrm{~d}_{1}=5 \\
& \mathrm{~d}_{2}=-5 \\
& \mathrm{~d}_{3}=10 / 3 \\
& \mathrm{~d}_{4}=-5 / 4 \\
& \mathrm{~d}_{5}=1 / 5
\end{aligned}
$$

implicando, finalmente, no seguinte operador de discretização:

$$
\Delta \mathrm{t} . \dot{\mathrm{x}}_{\mathrm{i}}=-\frac{137}{60} \mathrm{x}_{\mathrm{i}}+5 \mathrm{x}_{\mathrm{i}+1}-5 \mathrm{x}_{\mathrm{i}+2}+\frac{10}{3} \mathrm{x}_{\mathrm{i}+3}-\frac{5}{4} \mathrm{x}_{\mathrm{i}+4}+\frac{1}{5} \mathrm{x}_{\mathrm{i}+5}
$$

Apresenta-se no que se segue um exemplo de aplicação típico, como aqueles já mostrados nos capítulos precedentes.

\subsection{APLICAÇÃO PARA FILTRO DE DOIS PÓLOS}

Com esse algoritmo desenvolvido com quadratura numérica de ordem mais elevada foi processado o sinal $x(t)=\operatorname{sen}(20 t)$ considerando-se freqüência

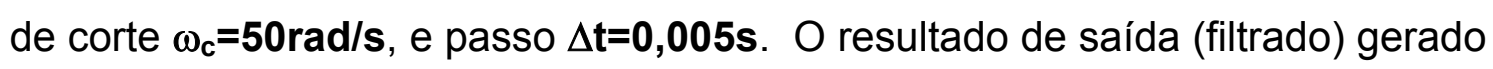
pelo algoritmo encontra-se na forma de gráfico conforme ilustrado na figura 6.1 : 


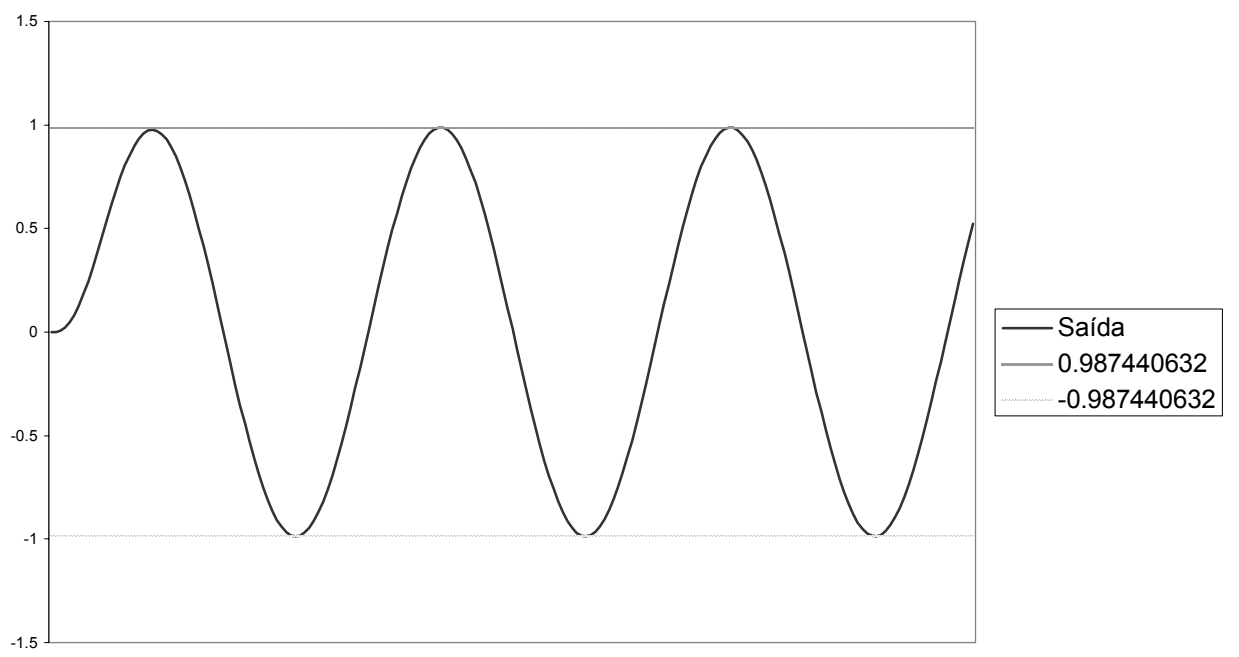

Fig. 6.1 - Saída do algoritmo hermitiano para baixa freqüência

Adotando-se agora uma entrada $\mathbf{x}(\mathbf{t})=\operatorname{sen}(\mathbf{7 0 t})$, o algoritmo fornece:

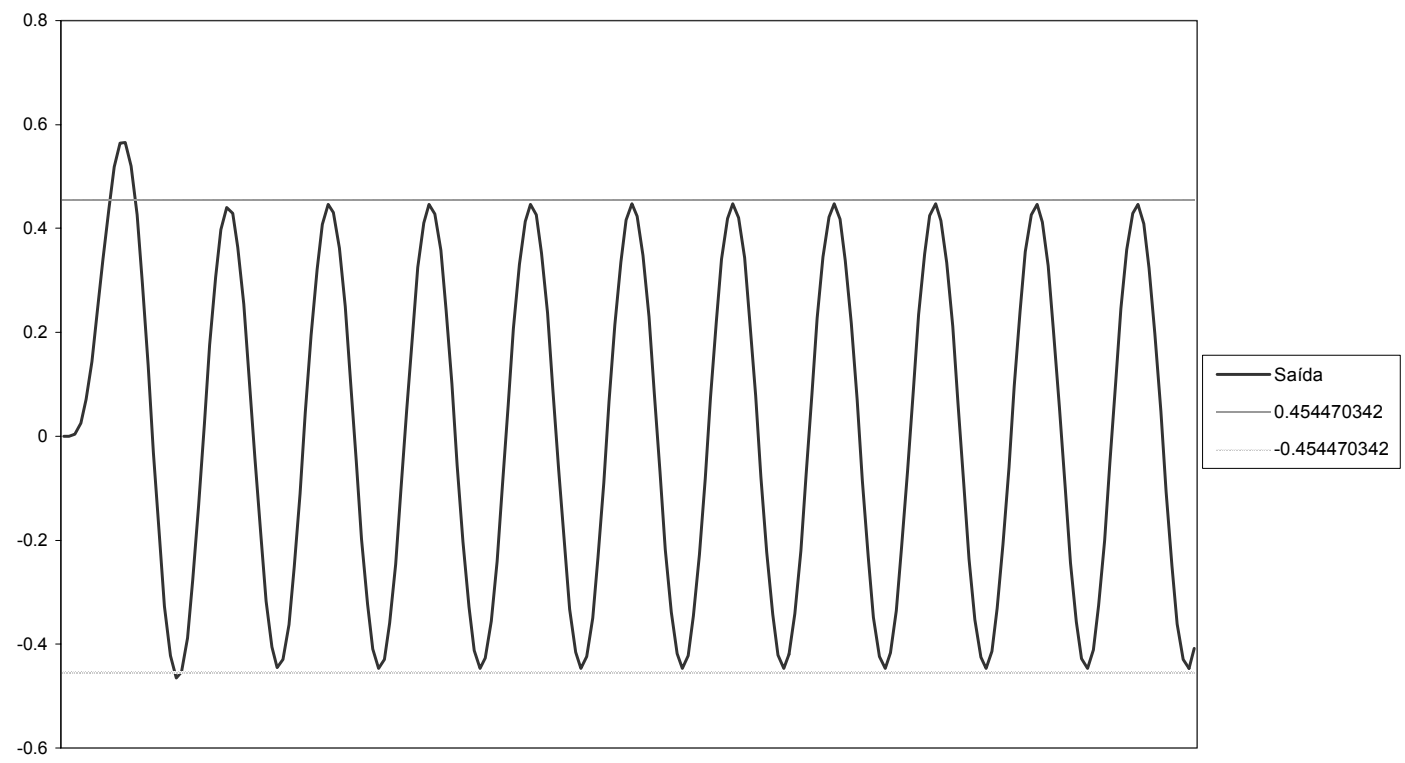

Fig. 6.2 - Saída do algoritmo hermitiano para alta freqüência

finalizando-se dessa forma o estudo do operador hermitiano de quadratura numérica mais elevada para filtro de Butterworth de dois pólos. 


\subsection{ALGORITMO HERMITIANO PARA FILTRO DE BUTTERWOTH DE ORDEM GENÉRICA}

Visando utilizar o integrador hermitiano para a resolução das demais equações diferenciais de filtragem, é necessário inicialmente recorrer-se à igualdade matricial disposta em (5.31), ou seja:

$$
\{\dot{\mathrm{Y}}\}_{\mathrm{i}}=[\mathrm{A}]\{\mathrm{Y}\}_{\mathrm{i}}+\{\mathrm{X}\}_{\mathrm{i}}
$$

onde a matriz $[\mathrm{A}]$ e os vetores apresentam a seguinte redação:

$$
[\mathbf{A}]=\left[\begin{array}{ccccccc}
0 & 1 & 0 & \cdots & 0 & 0 & 0 \\
0 & 0 & 1 & \cdots & 0 & 0 & 0 \\
\vdots & \vdots & \vdots & \ddots & \vdots & \vdots & \vdots \\
0 & 0 & 0 & \cdots & 1 & 0 & 0 \\
0 & 0 & 0 & \cdots & 0 & 1 & 0 \\
0 & 0 & 0 & \cdots & 0 & 0 & 1 \\
-\frac{\mathbf{a}_{0}}{\mathbf{a}_{\mathbf{N}}} & -\frac{\mathbf{a}_{1}}{\mathbf{a}_{\mathbf{N}}} & -\frac{\mathbf{a}_{3}}{\mathbf{a}_{\mathbf{N}}} & \cdots & -\frac{\mathbf{a}_{\mathbf{N}-3}}{\mathbf{a}_{\mathbf{N}}} & -\frac{\mathbf{a}_{\mathbf{N}-2}}{\mathbf{a}_{\mathbf{N}}} & -\frac{\mathbf{a}_{\mathbf{N}-1}}{\mathbf{a}_{\mathbf{N}}}
\end{array}\right]
$$

$$
\{Y\}_{i}=\left\{\begin{array}{c}
\mathrm{dy} / \mathrm{dt} \\
\mathrm{d}^{2} \mathrm{y} / \mathrm{dt}^{2} \\
\mathrm{~d}^{(\mathrm{N}-3)} \mathrm{y} / \mathrm{dt}^{(\mathrm{N}-3)} \\
\mathrm{d}^{(\mathrm{N}-2)} \mathrm{y} / \mathrm{dt}^{(\mathrm{N}-2)} \\
\mathrm{d}^{(\mathrm{N}-1)} \mathrm{y} / \mathrm{dt}^{(\mathrm{N}-1)}
\end{array}\right\}
$$

$$
\{X\}_{i}=\left\{\begin{array}{c}
0 \\
0 \\
\vdots \\
0 \\
\frac{b_{0}}{a_{N}} x
\end{array}\right\}_{i}
$$

Por outro lado, a derivação de (6.26) implica em: 


$$
\{\ddot{\mathrm{Y}}\}_{\mathrm{i}}=[\mathrm{A}]\{\dot{\mathrm{Y}}\}_{\mathrm{i}}+\{\dot{\mathrm{X}}\}_{\mathrm{i}}
$$

ou ainda, tendo-se em vista novamente (6.26):

$$
\{\ddot{\mathrm{Y}}\}_{\mathrm{i}}=[\mathrm{A}]^{2}\{\mathrm{Y}\}_{\mathrm{i}}+[\mathrm{A}]\{\mathrm{X}\}_{\mathrm{i}}+\{\dot{\mathrm{X}}\}_{\mathrm{i}}
$$

sendo que em termos genéricos a redação fica:

$$
\left.\frac{\mathrm{d}^{\mathrm{N}}\{\mathrm{Y}\}}{\mathrm{dt}^{\mathrm{N}}}\right|_{\mathrm{i}}=[\mathrm{A}]^{\mathrm{N}}\{\mathrm{Y}\}_{\mathrm{i}}+\left.\sum_{\mathrm{k}=0}^{\mathrm{N}-1}[\mathrm{~A}]^{\mathrm{N}-1-\mathrm{k}} \frac{\mathrm{d}^{\mathrm{k}}\{\mathrm{X}\}}{\mathrm{dt}^{\mathrm{k}}}\right|_{\mathrm{i}}
$$

A substituição de (6.28) e (6.29) em (6.8) e (6.9) já convertidos para notação vetorial, conduz finalmente a:

$$
\begin{aligned}
& \left(12[\mathrm{I}]-6 \Delta \mathrm{t}[\mathrm{A}]+\Delta \mathrm{t}^{2}[\mathrm{~A}]^{2}\right)\{\mathrm{Y}\}_{\mathrm{i}+1}=\left(12[\mathrm{I}]+6 \Delta \mathrm{t}[\mathrm{A}]+\Delta \mathrm{t}^{2}[\mathrm{~A}]^{2}\right)\{\mathrm{Y}\}_{\mathrm{i}}+ \\
& \left(6 \Delta \mathrm{t}+\Delta \mathrm{t}^{2}[\mathrm{~A}]\right)\{\mathrm{X}\}_{\mathrm{i}}+\left(6 \Delta \mathrm{t}-\Delta \mathrm{t}^{2}[\mathrm{~A}]\right)\{\mathrm{X}\}_{i+1}+\Delta \mathrm{t}^{2}\{\dot{\mathrm{X}}\}_{\mathrm{i}}-\Delta \mathrm{t}^{2}\{\dot{\mathrm{X}}\}_{i+1}
\end{aligned}
$$

que pode ser disposta na sua forma resumida:

$$
[\mathrm{G}]\{\mathrm{Y}\}_{\mathrm{i}+1}=\left[\mathrm{G}^{*}\right]\{\mathrm{Y}\}_{\mathrm{i}}+\{\mathrm{F}\}_{\mathrm{i}}
$$

onde:

$$
\begin{gathered}
{[\mathrm{G}]=12[\mathrm{I}]-6 \Delta \mathrm{t}[\mathrm{A}]+\Delta \mathrm{t}^{2}[\mathrm{~A}]^{2}} \\
{\left[\mathrm{G}^{*}\right]=12[\mathrm{I}]+6 \Delta \mathrm{t}[\mathrm{A}]+\Delta \mathrm{t}^{2}[\mathrm{~A}]^{2}} \\
\{\mathrm{~F}\}_{\mathrm{i}}=\left(6 \Delta \mathrm{t}+\Delta \mathrm{t}^{2}[\mathrm{~A}]\right)\{\mathrm{X}\}_{\mathrm{i}}+\left(6 \Delta \mathrm{t}-\Delta \mathrm{t}^{2}[\mathrm{~A}]\right)\{\mathrm{X}\}_{\mathrm{i}+1}+\Delta \mathrm{t}^{2}\{\dot{\mathrm{X}}\}_{\mathrm{i}}-\Delta \mathrm{t}^{2}\{\dot{\mathrm{X}}\}_{\mathrm{i}+1}
\end{gathered}
$$

generalizando-se assim o operador hermitiano para um filtro de Butterworth com qualquer número de pólos, e com convergência de qualquer ordem.

\subsection{VERIFICAÇÃO DA EFICIÊNCIA DO FILTRO UTILIZANDO NÚMERO DE PÓLOS MAIS ELEVADOS}

De maneira similar ao já exposto no capítulo anterior, a exemplificação do algoritmo agora desenvolvido toma por base o filtro de Butterworth com freqüência de corte $\omega_{\mathrm{c}}=50 \mathrm{rad} / \mathrm{s}$ e discretização $\Delta \mathrm{t}=\mathbf{0 , 0 1 \mathrm { s }}$, sendo considerado o 
sinal de entrada $x(t)=\operatorname{sen}(30 t)+0,3 \operatorname{sen}(70 t)$. Os resultados estão lançados nos gráficos das figuras que se seguem.

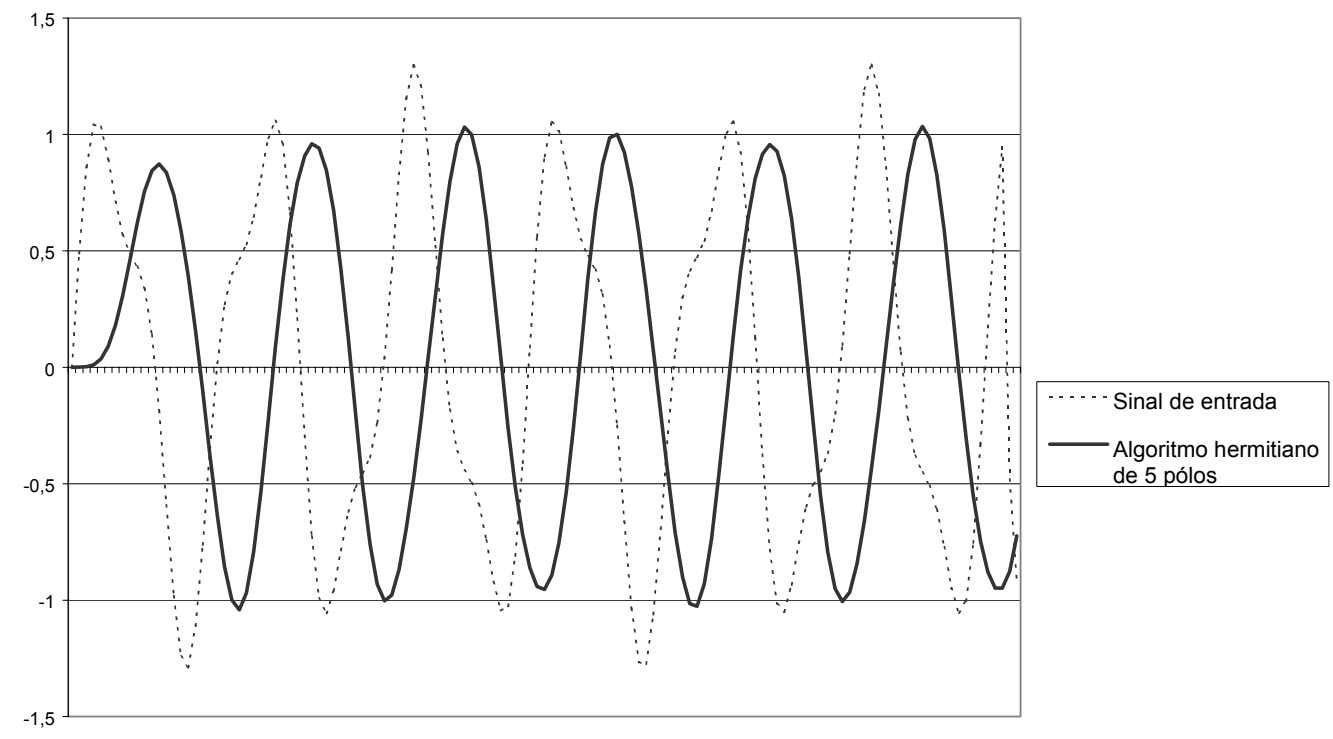

Figura 6.3 - Análise do filtro hermitiano de cinco pólos

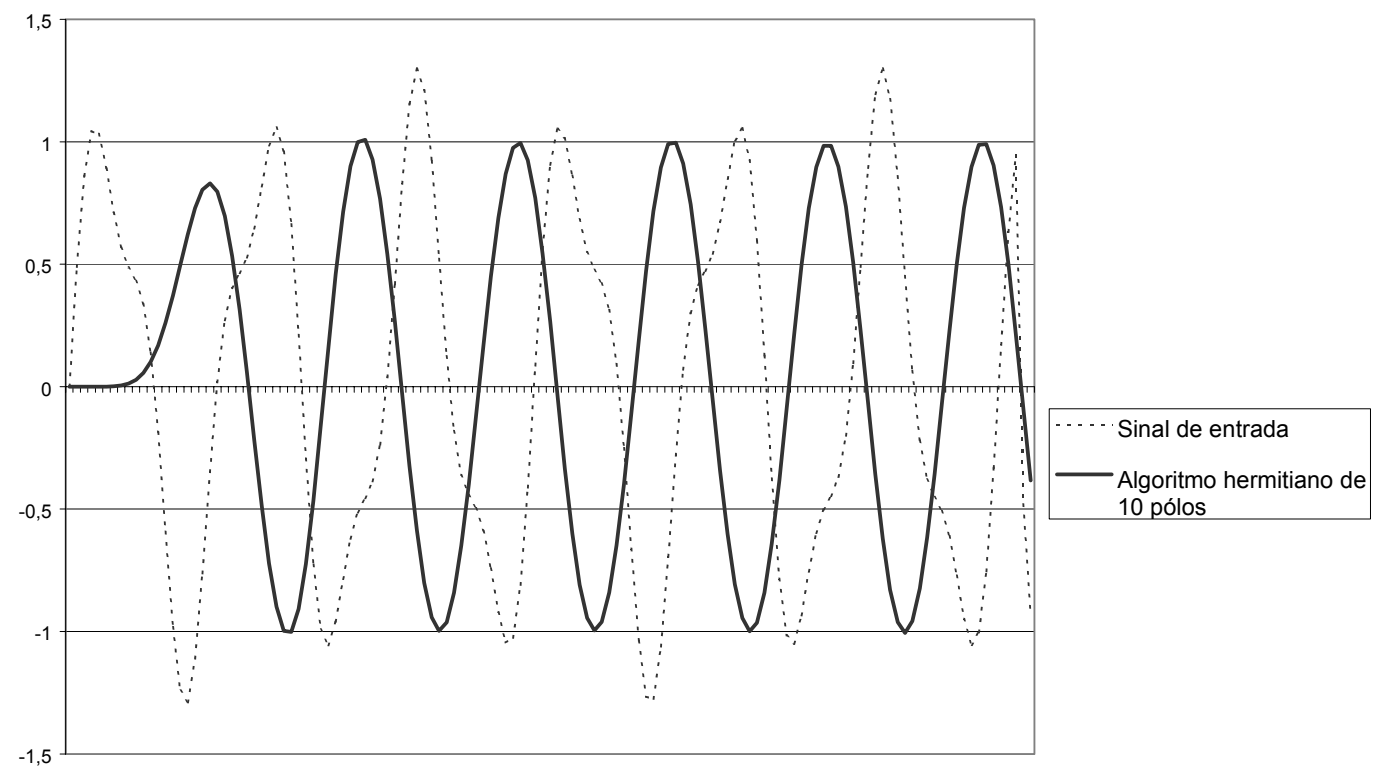

Figura 6.4 - Análise do filtro hermitiano de dez pólos 


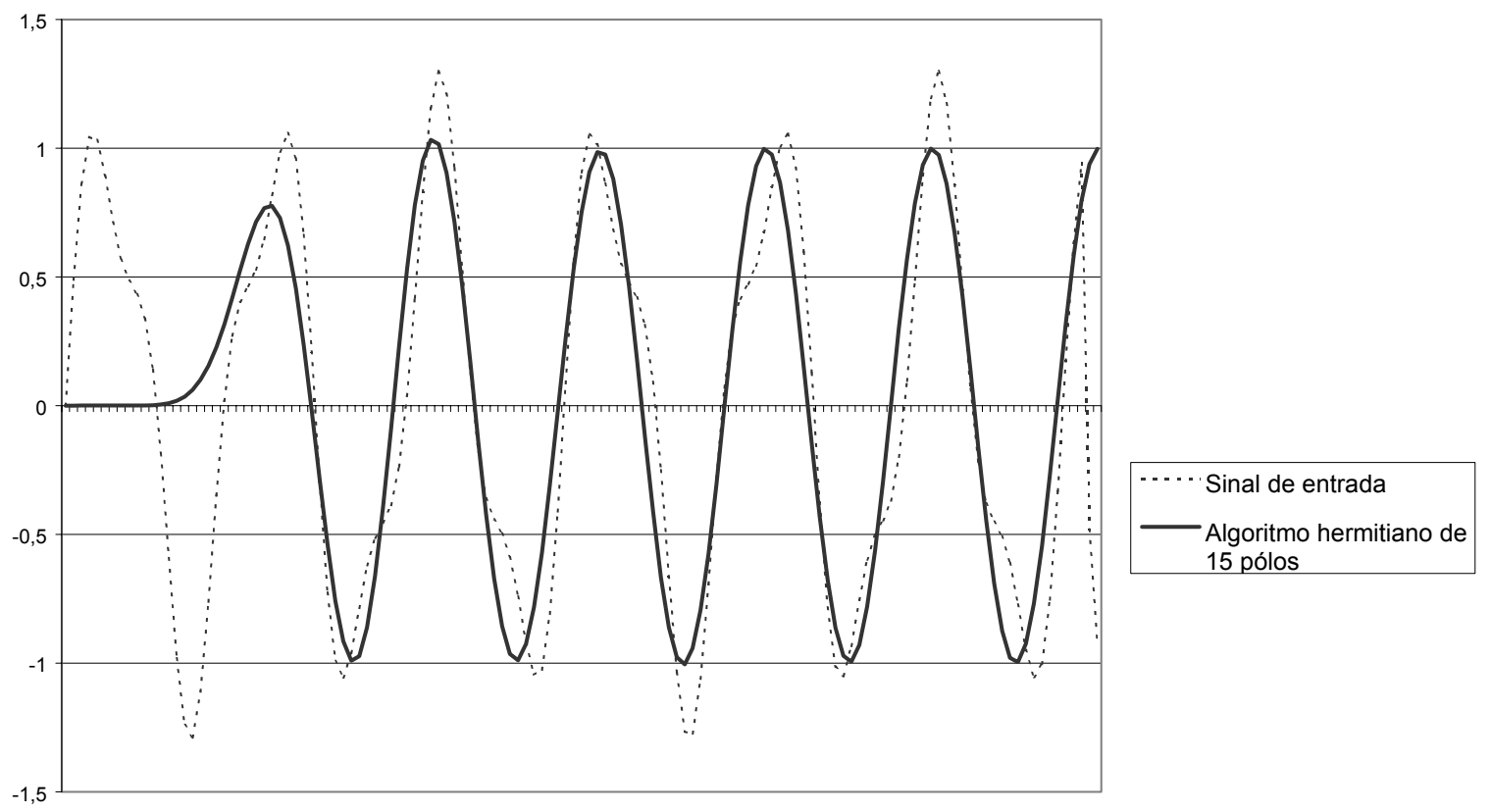

Figura 6.5 - Análise do filtro hermitiano de quinze pólos

Cumpre assinalar que a resposta é, para as discretizações consideradas, idêntica à resultante da aplicação do algoritmo trapezoidal na escala aqui empregada. Todavia, no próximo capítulo a questão da precisão só pode ser melhor avaliada confrontando-se resultados numéricos. 


\section{Capítulo VII}

\section{ANÁLISE COMPARATIVA DOS ALGORITMOS}

\subsection{INTRODUÇÃO}

Este capítulo destina-se a comparar os resultados obtidos com cada um dos algoritmos estudados, considerando diversos sinais de entrada, diversos números de pólos, sendo estudando o erro relativo nos diversos ensaios numéricos.

Como muito bem evidenciado, o erro encontrado com o operador hermitiano é realmente sensivelmente menor que o verificado com o algoritmo clássico trapezoidal. Vale registrar que os algoritmos aqui utilizados foram desenvolvidos e implementados em linguagem FORTRAN.

O comportamento do filtro digital para um sinal de entrada afetado por amortecido muito comum na experimentação estrutural (vibração livre), assunto que os engenheiros elétricos geralmente não abordam, é também colocado em destaque.

Mais uma vez, é importante registrar que o fator principal para uma boa filtragem não é só a precisão do algoritmo, mas sim também a consideração de um número elevado de pólos no filtro digital, inclusive considerando o erro de leitura do aparelho de mensuração através de um acréscimo randômico. 


\subsection{PRIMEIRO EXEMPLO: COMPARAÇÃO DE ENTRADA COMPOSTA POR UMA ÚNICA SENÓIDE}

A entrada considerada no primeiro estudo é o caso de uma única senóide, ou seja, $x(t)=\operatorname{sen}\left(\omega_{0} t\right)$. Neste exemplo, estipula-se uma freqüência de corte $\omega_{\mathrm{c}}=50 \mathrm{rad} / \mathrm{s}$, com $\omega_{0}$ adquirindo valores de 30 e $70 \mathrm{rad} / \mathrm{s}$. O estudo em questão leva

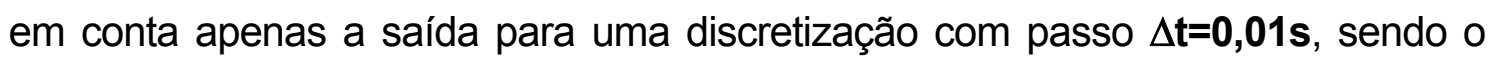
filtro tomado com dois pólos. Procurando-se eliminar do estudo a parcela transitória do sistema, a comparação se dá para valores já com um certo tempo decorrido.

Os resultados de interesse nesse caso acham-se arrolados na tabela 7.1.

\begin{tabular}{|c|c|c|c|c|c|c|c|}
\hline \multirow{2}{*}{$t(s)$} & \multirow{2}{*}{$\mathrm{i}$} & \multicolumn{2}{|c|}{ Trapezoidal } & \multicolumn{2}{c|}{ Operador hermitiano } & \multicolumn{2}{c|}{ Resultado teórico } \\
\cline { 3 - 8 } & & $\omega_{0}=30 \mathrm{rad} / \mathrm{s}$ & $\omega_{0}=70 \mathrm{rad} / \mathrm{s}$ & $\omega_{0}=30 \mathrm{rad} / \mathrm{s}$ & $\omega_{0}=70 \mathrm{rad} / \mathrm{s}$ & $\omega_{0}=30 \mathrm{rad} / \mathrm{s}$ & $\omega_{0}=70 \mathrm{rad} / \mathrm{s}$ \\
\hline 0,50 & 50 & 0,936973 & 0,423925 & 0,939050 & 0,453721 & 0,939094 & 0,454452 \\
\hline 0,51 & 51 & 0,914395 & 0,340356 & 0,914268 & 0,349738 & 0,914310 & 0,350217 \\
\hline 0,52 & 52 & 0,810137 & 0,096713 & 0,807813 & 0,081249 & 0,807852 & 0,081269 \\
\hline 0,53 & 53 & 0,633511 & $-0,192416$ & 0,629196 & $-0,225471$ & 0,629232 & $-0,225901$ \\
\hline 0,54 & 54 & 0,400296 & $-0,391049$ & 0,394370 & $-0,426168$ & 0,394404 & $-0,426826$ \\
\hline 0,55 & 55 & 0,131324 & $-0,405765$ & 0,124314 & $-0,426450$ & 0,124346 & $-0,427008$ \\
\hline 0,56 & 56 & $-0,149379$ & $-0,229643$ & $-0,156851$ & $-0,226185$ & $-0,156820$ & $-0,226362$ \\
\hline 0,57 & 57 & $-0,416738$ & 0,054483 & $-0,424009$ & 0,080440 & $-0,423978$ & 0,080746 \\
\hline 0,58 & 58 & $-0,646872$ & 0,312985 & $-0,653294$ & 0,349213 & $-0,653263$ & 0,349878 \\
\hline 0,59 & 59 & $-0,819222$ & 0,424285 & $-0,824226$ & 0,453728 & $-0,824194$ & 0,454456 \\
\hline 0,60 & 60 & $-0,918394$ & 0,336038 & $-0,921537$ & 0,344828 & $-0,921502$ & 0,345297 \\
\hline
\end{tabular}

Tabela 7.1 - Valores de $y_{i}$ dos diferentes algoritmos para $\Delta \mathbf{t}=\mathbf{0 , 0 1 s}$

Tabelando-se então o erro, que é a diferença entre o valor teórico da saída e o valor obtido pelo algoritmo, tem-se os resultados como lançados na tabela 7.2:

\begin{tabular}{|c|c|c|c|c|c|}
\hline \multirow{2}{*}{$t(s)$} & \multirow{2}{*}{$i$} & \multicolumn{2}{|c|}{ Trapezoidal } & \multicolumn{2}{c|}{ Operador hermitiano } \\
\cline { 3 - 6 } & & $\omega_{0}=30 \mathrm{rad} / \mathrm{s}$ & $\omega_{0}=70 \mathrm{rad} / \mathrm{s}$ & $\omega_{0}=30 \mathrm{rad} / \mathrm{s}$ & $\omega_{0}=70 \mathrm{rad} / \mathrm{s}$ \\
\hline 0,50 & 50 & 0,002121 & 0,030527 & 0,000005 & 0,000691 \\
\hline 0,51 & 51 & $-0,000085$ & 0,009861 & 0,000002 & 0,000438 \\
\hline 0,52 & 52 & $-0,002284$ & $-0,015444$ & $-0,000001$ & $-0,000020$ \\
\hline 0,53 & 53 & $-0,004279$ & $-0,033485$ & $-0,000004$ & $-0,000470$ \\
\hline 0,54 & 54 & $-0,005892$ & $-0,035777$ & $-0,000006$ & $-0,000698$ \\
\hline 0,55 & 55 & $-0,006978$ & $-0,021243$ & $-0,000008$ & $-0,000598$ \\
\hline 0,56 & 56 & $-0,007441$ & 0,003282 & $-0,000009$ & $-0,000217$ \\
\hline 0,57 & 57 & $-0,007240$ & 0,026263 & $-0,000009$ & 0,000266 \\
\hline 0,58 & 58 & $-0,006391$ & 0,036893 & $-0,000009$ & 0,000624 \\
\hline 0,59 & 59 & $-0,004972$ & 0,030171 & $-0,000008$ & 0,000689 \\
\hline 0,60 & 60 & $-0,003109$ & 0,009259 & $-0,000006$ & 0,000429 \\
\hline
\end{tabular}

Tabela 7.2 - Erros locais dos algoritmos para $\Delta \mathrm{t}=\mathbf{0 , 0 1 \mathrm { s }}$ 
Nota-se aqui claramente, que o erro local do algoritmo trapezoidal é bem maior que o erro local do algoritmo hermitiano, como deveria de ser esperado. Percebe-se ainda que quanto maior a freqüência do sinal de entrada, maior o erro verificado no sinal filtrado. Por se tratar de matrizes pequenas, o esforço computacional é mínimo, onde o resultado final é fornecido instantaneamente.

\subsection{SEGUNDO EXEMPLO: COMPARAÇÃO DO COMPORTAMENTO DOS ALGORITMOS PARA DIFERENTES VALORES DE $\Delta t$}

Tendo-se em vista estudar agora a influência do passo de tempo $\Delta$ t na integração com os dois tipos de algoritmo, exemplifica-se aqui o caso de um sinal composto por duas senóides, sendo que o de baixa freqüência será considerado como sinal desejado (que deve passar) e o de alta freqüência será considerado como sinal indesejado (que deve ser retido). O sinal em questão e:

$$
x(t)=\operatorname{sen}(30 t)+0,3 \operatorname{sen}(70 t)
$$

sendo considerada a freqüência de corte $\omega_{c}=50 \mathrm{rad} / \mathrm{s}$. As tabelas $7.3,7.4$ e 7.5 mostram os resultados obtidos com os algoritmos trapezoidal e hermitiano, para um filtro de dois pólos com discretizações no tempo com passos de $\Delta t=0,005 s$, $\Delta t=0,01 s$ e $\Delta t=0,02 s$, respectivamente. A comparação com o resultado teórico e seus respectivos erros locais é também aí indicada.

\begin{tabular}{|c|c|c|c|c|c|c|}
\hline $\mathrm{t}(\mathrm{s})$ & $\mathrm{i}$ & $\begin{array}{c}\text { Algoritmo } \\
\text { Trapezoidal }\end{array}$ & $\begin{array}{c}\text { Algoritmo } \\
\text { Hermitiano }\end{array}$ & $\begin{array}{c}\text { Resultado } \\
\text { Teórico }\end{array}$ & $\begin{array}{c}\text { Erro Local } \\
\text { Trapezoidal }\end{array}$ & $\begin{array}{c}\text { Erro Local } \\
\text { Hermitiano }\end{array}$ \\
\hline 2,000 & 400 & 0,5088771 & 0,5086904 & 0,5086911 & $-0,0001860$ & 0,0000007 \\
\hline 2,005 & 401 & 0,4346363 & 0,4350738 & 0,4350764 & 0,0004400 & 0,0000025 \\
\hline 2,010 & 402 & 0,3495061 & 0,3503734 & 0,3503774 & 0,0008713 & 0,0000040 \\
\hline 2,015 & 403 & 0,2509394 & 0,2519742 & 0,2519792 & 0,0010397 & 0,0000050 \\
\hline 2,020 & 404 & 0,1373685 & 0,1382760 & 0,1382813 & 0,0009128 & 0,0000052 \\
\hline 2,025 & 405 & 0,0086985 & 0,0091910 & 0,0091958 & 0,0004974 & 0,0000048 \\
\hline 2,030 & 406 & $-0,1333696$ & $-0,1335339$ & $-0,1335302$ & $-0,0001606$ & 0,0000037 \\
\hline 2,035 & 407 & $-0,2852329$ & $-0,2862166$ & $-0,2862145$ & $-0,0009816$ & 0,0000021 \\
\hline 2,040 & 408 & $-0,4415178$ & $-0,4433803$ & $-0,4433801$ & $-0,0018623$ & 0,0000002 \\
\hline 2,045 & 409 & $-0,5954384$ & $-0,5981248$ & $-0,5981266$ & $-0,0026882$ & $-0,0000018$ \\
\hline 2,050 & 410 & $-0,7393462$ & $-0,7426902$ & $-0,7426938$ & $-0,0033476$ & $-0,0000036$ \\
\hline
\end{tabular}

Tabela 7.3 - Erros locais dos algoritmos para $\Delta \mathrm{t}=\mathbf{0 , 0 0 5 s}$ 


\begin{tabular}{|c|c|c|c|c|c|c|}
\hline$t(s)$ & $\mathrm{i}$ & $\begin{array}{c}\text { Algoritmo } \\
\text { Trapezoidal }\end{array}$ & $\begin{array}{c}\text { Algoritmo } \\
\text { Hermitiano }\end{array}$ & $\begin{array}{c}\text { Resultado } \\
\text { Teórico }\end{array}$ & $\begin{array}{c}\text { Erro Local } \\
\text { Trapezoidal }\end{array}$ & $\begin{array}{c}\text { Erro Local } \\
\text { Hermitiano }\end{array}$ \\
\hline 2,000 & 200 & 0,5097683 & 0,5087050 & 0,5086911 & $-0,0010772$ & $-0,0000138$ \\
\hline 2,010 & 201 & 0,3470891 & 0,3502552 & 0,3503774 & 0,0032882 & 0,0001222 \\
\hline 2,020 & 202 & 0,1346076 & 0,1380831 & 0,1382813 & 0,0036736 & 0,0001982 \\
\hline 2,030 & 203 & $-0,1331039$ & $-0,1337079$ & $-0,1335302$ & $-0,0004263$ & 0,0001777 \\
\hline 2,040 & 204 & $-0,4362191$ & $-0,4434502$ & $-0,4433801$ & $-0,0071610$ & 0,0000701 \\
\hline 2,050 & 205 & $-0,7295064$ & $-0,7426198$ & $-0,7426938$ & $-0,0131875$ & $-0,0000741$ \\
\hline 2,060 & 206 & $-0,9549078$ & $-0,9699793$ & $-0,9701660$ & $-0,0152582$ & $-0,0001867$ \\
\hline 2,070 & 207 & $-1,0617093$ & $-1,0732806$ & $-1,0734948$ & $-0,0117854$ & $-0,0002142$ \\
\hline 2,080 & 208 & $-1,0254530$ & $-1,0289530$ & $-1,0290959$ & $-0,0036428$ & $-0,0001428$ \\
\hline 2,090 & 209 & $-0,8571378$ & $-0,8509550$ & $-0,8509601$ & 0,0061777 & $-0,0000051$ \\
\hline 2,100 & 210 & $-0,5985870$ & $-0,5848240$ & $-0,5846888$ & 0,0138982 & 0,0001352 \\
\hline
\end{tabular}

Tabela 7.4 - Erros locais dos algoritmos para $\Delta \mathrm{t}=\mathbf{0 , 0 1 s}$

\begin{tabular}{|c|c|c|c|c|c|c|}
\hline $\mathrm{t}(\mathrm{s})$ & $\mathrm{i}$ & $\begin{array}{c}\text { Algoritmo } \\
\text { Trapezoidal }\end{array}$ & $\begin{array}{c}\text { Algoritmo } \\
\text { Hermitiano }\end{array}$ & $\begin{array}{c}\text { Resultado } \\
\text { Teórico }\end{array}$ & $\begin{array}{c}\text { Erro Local } \\
\text { Trapezoidal }\end{array}$ & $\begin{array}{c}\text { Erro Local } \\
\text { Hermitiano }\end{array}$ \\
\hline 2,000 & 100 & 0,5186370 & 0,4976238 & 0,5086911 & $-0,0099459$ & 0,0110674 \\
\hline 2,020 & 101 & 0,1242062 & 0,1310142 & 0,1382813 & 0,0140750 & 0,0072670 \\
\hline 2,040 & 102 & $-0,4193104$ & $-0,4346896$ & $-0,4433801$ & $-0,0240697$ & $-0,0086905$ \\
\hline 2,060 & 103 & $-0,9100579$ & $-0,9596002$ & $-0,9701660$ & $-0,0601081$ & $-0,0105657$ \\
\hline 2,080 & 104 & $-1,0090537$ & $-1,0337195$ & $-1,0290959$ & $-0,0200422$ & 0,0046236 \\
\hline 2,100 & 105 & $-0,6367483$ & $-0,5963864$ & $-0,5846888$ & 0,0520595 & 0,0116976 \\
\hline 2,120 & 106 & $-0,0754633$ & $-0,0151865$ & $-0,0160847$ & 0,0593785 & $-0,0008982$ \\
\hline 2,140 & 107 & 0,3820011 & 0,3990591 & 0,3870819 & 0,0050808 & $-0,0119772$ \\
\hline 2,160 & 108 & 0,6952230 & 0,6798132 & 0,6769332 & $-0,0182897$ & $-0,0028799$ \\
\hline 2,180 & 109 & 0,8920949 & 0,8973578 & 0,9088145 & 0,0167197 & 0,0114567 \\
\hline 2,200 & 110 & 0,8311364 & 0,8547581 & 0,8619961 & 0,0308598 & 0,0072380 \\
\hline
\end{tabular}

Tabela 7.5 - Erros locais dos algoritmos para $\Delta \mathrm{t}=\mathbf{0 , 0 2 \mathrm { s }}$

Percebe-se nesse exemplo que o algoritmo hermitiano, embora seja derivado de uma quadratura numérica superior, apresenta aqui a mesma instabilidade observada para o algoritmo trapezoidal, conforme o passo de tempo se aproxima do período do sinal. Este fenômeno, conhecido por aliasing, gera, como sabido, uma máscara para o sinal de entrada, tornando-se assim inviável qualquer algoritmo de filtragem, pois é uma questão ligada a insuficiência da discretização do sinal de entrada. Para solucionar este problema, é necessário processar-se inicialmente o sinal de entrada mediante um filtro do tipo anti-aliasing. Por exemplo, se for adotado no caso em estudo uma discretização com passo $\Delta t=0,04 s$, os resultados já são os mostrados na tabela 6 exibida no que se segue: 


\begin{tabular}{|c|c|c|c|c|c|c|}
\hline $\mathrm{t}(\mathrm{s})$ & $\mathrm{i}$ & $\begin{array}{c}\text { Algoritmo } \\
\text { Trapezoidal }\end{array}$ & $\begin{array}{c}\text { Algoritmo } \\
\text { Hermitiano }\end{array}$ & $\begin{array}{c}\text { Resultado } \\
\text { Teórico }\end{array}$ & $\begin{array}{c}\text { Erro Local } \\
\text { Trapezoidal }\end{array}$ & $\begin{array}{c}\text { Erro Local } \\
\text { Hermitiano }\end{array}$ \\
\hline 2,000 & 50 & 0,6148278 & 0,8186145 & 0,5086911 & $-0,1061366$ & $-0,3099233$ \\
\hline 2,040 & 51 & $-0,3782514$ & $-0,6609445$ & $-0,4433801$ & $-0,0651287$ & 0,2175643 \\
\hline 2,080 & 52 & $-0,9121021$ & $-0,8383014$ & $-1,0290959$ & $-0,1169937$ & $-0,1907945$ \\
\hline 2,120 & 53 & $-0,2601429$ & $-0,1289265$ & $-0,0160847$ & 0,2440582 & 0,1128418 \\
\hline 2,160 & 54 & 0,7040956 & 0,6291680 & 0,6769332 & $-0,0271624$ & 0,0477653 \\
\hline 2,200 & 55 & 0,7844948 & 0,9852624 & 0,8619961 & 0,0775013 & $-0,1232663$ \\
\hline 2,240 & 56 & $-0,1426216$ & $-0,5539063$ & $-0,3813203$ & $-0,2386987$ & 0,1725859 \\
\hline 2,280 & 57 & $-0,8886307$ & $-0,5833222$ & $-0,8735222$ & 0,0151085 & $-0,2902000$ \\
\hline 2,320 & 58 & $-0,4928593$ & $-0,7439595$ & $-0,4216894$ & 0,0711699 & 0,3222700 \\
\hline 2,360 & 59 & 0,5161616 & 0,8899196 & 0,6233648 & 0,1072032 & $-0,2665547$ \\
\hline 2,400 & 60 & 0,8872123 & 0,6702400 & 0,9389185 & 0,0517062 & 0,2686785 \\
\hline
\end{tabular}

Tabela 7.6 - Erros locais dos algoritmos para $\Delta \mathbf{t}=\mathbf{0 , 0 4 s}$

Percebe-se, pois, que nesse caso os erros locais do algoritmo hermitiano se mostram geralmente maiores que os resultantes com o algoritmo trapezoidal. Todavia, é necessário ter-se em vista que o período do sinal de maior freqüência é dado por:

$$
\mathrm{T}=2 \pi / 70=0,0898 \mathrm{~s}
$$

podendo-se afirmar que um passo de tempo igual a 0,04s é quase a metade, pois, do seu período (discretização bastante pobre), implicando assim na ineficiência observada do filtro.

\subsection{TERCEIRO EXEMPLO: ANÁLISE DE ENTRADA AMORTECIDA}

No estudo da dinâmica das estruturas, o movimento livre apresenta componentes que descrevem movimento oscilatório amortecido. Esse caso, evidentemente, é bastante essencial para o estudo da filtragem dos sinais (deslocamentos, velocidade e aceleração) que descrevem os movimentos de uma estrutura. Considere-se então o caso de um sinal de entrada do tipo:

$$
x(t)=\operatorname{sen}(30 t) e^{-2 t}
$$

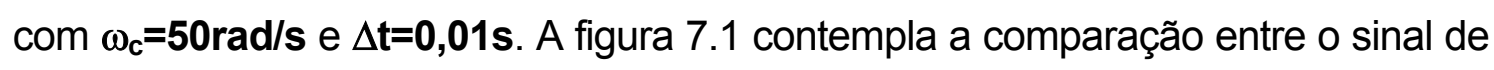
entrada (7.3) e o sinal de saída (filtrado), empregando-se, por exemplo, um filtro trapezoidal de dez pólos. No início do movimento, nota-se que a correspondência 
em amplitude não é obedecida devido ao tempo de resposta do sistema. Por outro lado, a partir do segundo ciclo em diante, observa-se que há sim uma correspondência na amplitude do sinal de entrada e do sinal de saída, denotandose uma certa defasagem.

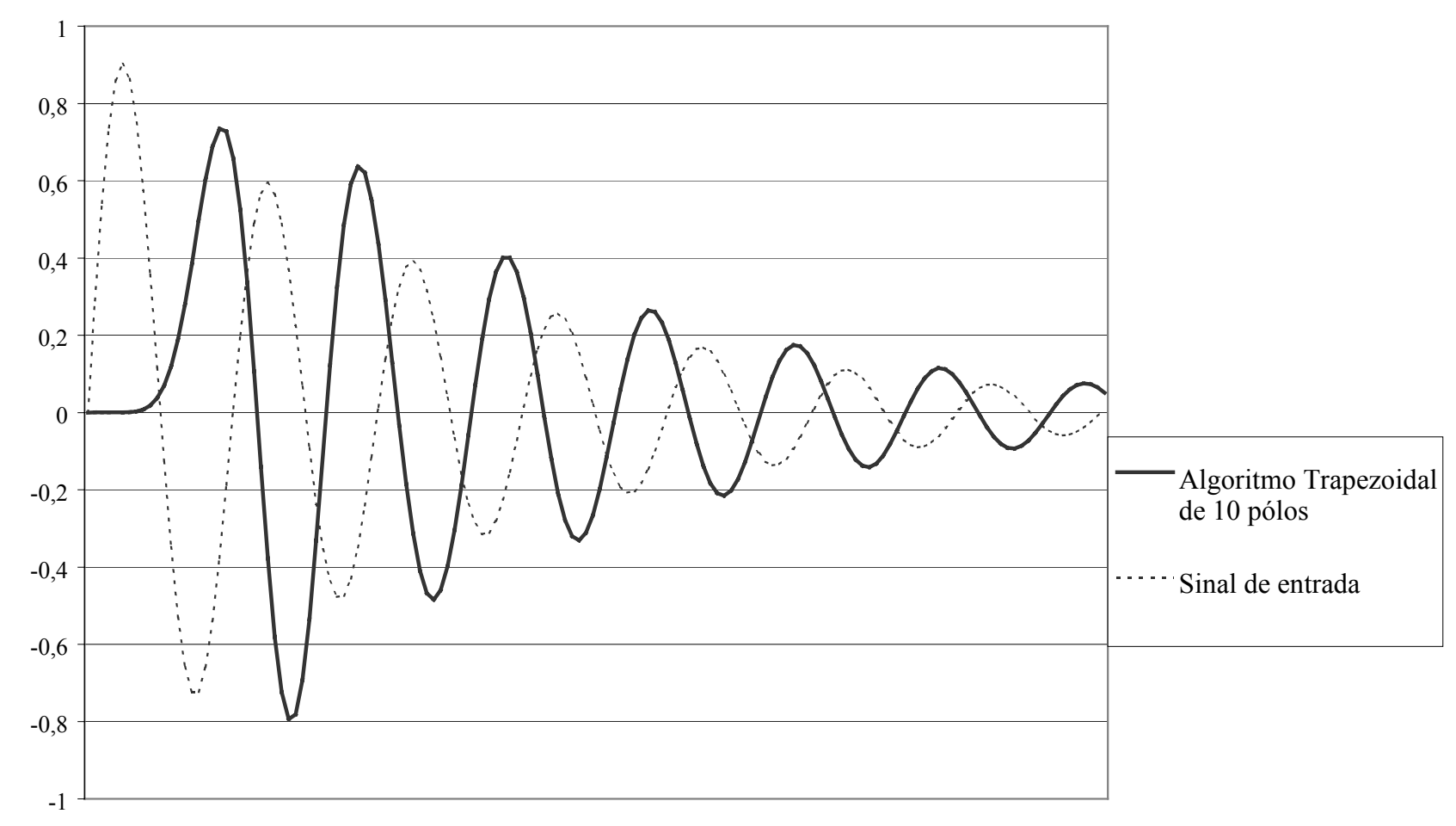

Fig. 7.1 - Análise de entrada exponencial no algoritmo trapezoidal de dez pólos

Contudo, pode-se afirmar que o filtro digital apresenta bom desempenho em casos de sinais contendo amortecimento, como o estudado. Isso ocorre porque a equação de filtragem tem como parâmetro principal de escala a freqüência do sinal de entrada.

\subsection{QUARTO EXEMPLO: ANÁLISE DE ENTRADA CONTENDO PARCELA RANDÔMICA}

Devido ao fato da coleta dos dados de entrada ser proveniente de uma mensuração, é interessante verificar o comportamento do algoritmo perante a presença de uma parcela aleatória no sinal de entrada, que procura simular toda a gama de erros gerados na mensuração. Admitindo então uma entrada do tipo: 


$$
x(t)=\operatorname{sen}(30 t)+\varepsilon(0,1)
$$

onde $\varepsilon$ representa uma parcela aleatória de valor máximo 0,1 , para um filtro de dois

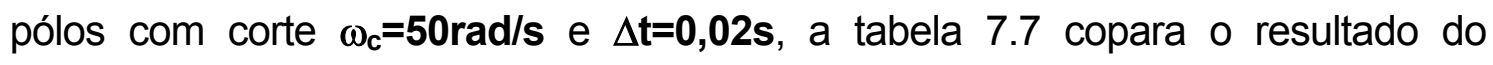
algoritmo trapezoidal, o resultado do algoritmo hermitiano e o resultado teórico, com os respectivos erros locais dos algoritmos.

\begin{tabular}{|c|c|c|c|c|c|}
\hline \multirow{2}{*}{$\mathrm{i}$} & \multicolumn{2}{|c|}{ Yi do algoritmo: } & \multirow{2}{*}{ Resultado } & \multicolumn{2}{c|}{ Erro local } \\
\cline { 7 - 7 } \cline { 6 - 6 } & Hermitiano & Trapezoidal & Teórico & Hermitiano & Trapezoidal \\
\hline 200 & $-0,282586027$ & $-0,3099134$ & $-0,2826348$ & $-0,0000488$ & 0,0272786 \\
\hline 201 & 0,273296109 & 0,20050626 & 0,2734604 & 0,0001643 & 0,0729542 \\
\hline 202 & 0,733712846 & 0,685483872 & 0,7340281 & 0,0003152 & 0,0485442 \\
\hline 203 & 0,937827373 & 0,94646643 & 0,9381786 & 0,0003512 & $-0,0082878$ \\
\hline 204 & 0,814336612 & 0,844372323 & 0,8145964 & 0,0002597 & $-0,0297760$ \\
\hline 205 & 0,40637944 & 0,418575098 & 0,4064522 & 0,0000727 & $-0,0121229$ \\
\hline 206 & $-0,143532967$ & $-0,14096523$ & $-0,1436775$ & $-0,0001445$ & $-0,0027122$ \\
\hline 207 & $-0,643300384$ & $-0,61347719$ & $-0,6436164$ & $-0,0003160$ & $-0,0301392$ \\
\hline 208 & $-0,918339673$ & $-0,87580235$ & $-0,9187216$ & $-0,0003820$ & $-0,0429193$ \\
\hline 209 & $-0,872571698$ & $-0,85718783$ & $-0,8728910$ & $-0,0003193$ & $-0,0157031$ \\
\hline 210 & $-0,52198453$ & $-0,52254391$ & $-0,5221344$ & $-0,0001498$ & 0,0004095 \\
\hline
\end{tabular}

Tabela 7.7 - Análise de entrada randômica com envoltória de $10 \%$ do valor

da amplitude do sinal de entrada

Percebe-se que o algoritmo hermitiano se comporta de forma excepcional perante um erro aleatório de $10 \%$ da amplitude. Supondo então o mesmo exemplo porém com um erro de $20 \%$ da amplitude, ou seja $\varepsilon(0,2)$, a tabela 7.8 compara os valores obtidos nos dois algoritmos:

\begin{tabular}{|c|c|c|c|c|c|}
\hline \multirow{2}{*}{$\mathrm{i}$} & \multicolumn{2}{|c|}{ Yi do algoritmo: } & Resultado & \multicolumn{2}{c|}{ Erro local } \\
\cline { 6 - 6 } & Hermitiano & Trapezoidal & Teórico & Hermitiano & Trapezoidal \\
\hline 200 & $-0,282632861$ & $-0,3222592$ & $-0,2826348$ & $-0,0000020$ & 0,0396244 \\
\hline 201 & 0,273249275 & 0,272187367 & 0,2734604 & 0,0002111 & 0,0012730 \\
\hline 202 & 0,733666012 & 0,789203516 & 0,7340281 & 0,0003621 & $-0,0551754$ \\
\hline 203 & 0,937780539 & 1,025659272 & 0,9381786 & 0,0003981 & $-0,0874807$ \\
\hline 204 & 0,814289778 & 0,935141511 & 0,8145964 & 0,0003066 & $-0,1205452$ \\
\hline 205 & 0,406332606 & 0,543742334 & 0,4064522 & 0,0001196 & $-0,1372902$ \\
\hline 206 & $-0,143579801$ & $-0,02683348$ & $-0,1436775$ & $-0,0000977$ & $-0,1168440$ \\
\hline 207 & $-0,643347218$ & $-0,5491421$ & $-0,6436164$ & $-0,0002692$ & $-0,0944743$ \\
\hline 208 & $-0,918386507$ & $-0,84030032$ & $-0,9187216$ & $-0,0003351$ & $-0,0784213$ \\
\hline 209 & $-0,872618532$ & $-0,82839273$ & $-0,8728910$ & $-0,0002724$ & $-0,0444982$ \\
\hline 210 & $-0,522031364$ & $-0,50873409$ & $-0,5221344$ & $-0,0001030$ & $-0,0134003$ \\
\hline
\end{tabular}

Tabela 7.8 - Análise de entrada randômica com envoltória de $20 \%$ do valor da amplitude do sinal de entrada 
Nota-se novamente o bom desempenho do algoritmo hermitiano. Na busca do valor limite de eficiência de filtragem do algoritmo hermitiano, a tabela 7.9 admite para o mesmo exemplo um erro de até $30 \%$ na leitura do aparelho:

\begin{tabular}{|c|c|c|c|c|c|}
\hline \multirow{2}{*}{$\mathrm{i}$} & \multicolumn{2}{|c|}{ Yi do algoritmo: } & Resultado & \multicolumn{2}{c|}{ Erro local } \\
\cline { 7 - 7 } \cline { 6 - 6 } & Hermitiano & Trapezoidal & Teórico & Hermitiano & Trapezoidal \\
\hline 200 & $-0,282656478$ & $-0,3339654$ & $-0,2826348$ & 0,0000216 & 0,0513306 \\
\hline 201 & 0,273225916 & 0,202841142 & 0,2734604 & 0,0002345 & 0,0706193 \\
\hline 202 & 0,733642489 & 0,75592873 & 0,7340281 & 0,0003856 & $-0,0219007$ \\
\hline 203 & 0,93775683 & 1,032592538 & 0,9381786 & 0,0004218 & $-0,0944139$ \\
\hline 204 & 0,81426597 & 0,959561831 & 0,8145964 & 0,0003304 & $-0,1449655$ \\
\hline 205 & 0,406308769 & 0,617420176 & 0,4064522 & 0,0001434 & $-0,2109680$ \\
\hline 206 & $-0,143603636$ & 0,078786053 & $-0,1436775$ & $-0,0000738$ & $-0,2224635$ \\
\hline 207 & $-0,643371044$ & $-0,51893862$ & $-0,6436164$ & $-0,0002454$ & $-0,1246778$ \\
\hline 208 & $-0,918410327$ & $-0,88306626$ & $-0,9187216$ & $-0,0003113$ & $-0,0356554$ \\
\hline 209 & $-0,87264235$ & $-0,85700615$ & $-0,8728910$ & $-0,0002486$ & $-0,0158848$ \\
\hline 210 & $-0,522055181$ & $-0,54887377$ & $-0,5221344$ & $-0,0000792$ & 0,0267394 \\
\hline
\end{tabular}

Tabela 7.9 - Análise de entrada randômica com envoltória de $30 \%$ do valor da amplitude do sinal de entrada

Admitindo $40 \%$ de erro, tem-se a tabela 7.10 :

\begin{tabular}{|c|c|c|c|c|c|}
\hline \multirow{2}{*}{$\mathrm{i}$} & \multicolumn{2}{|c|}{ Yi do algoritmo: } & Resultado & \multicolumn{2}{c|}{ Erro local } \\
\cline { 6 - 6 } & Hermitiano & Trapezoidal & Teórico & Hermitiano & Trapezoidal \\
\hline 200 & $-0,282574597$ & $-0,32214583$ & $-0,2826348$ & $-0,0000602$ & 0,0395110 \\
\hline 201 & 0,273224884 & 0,214650607 & 0,2734604 & 0,0002355 & 0,0588098 \\
\hline 202 & 0,733702487 & 0,668141946 & 0,7340281 & 0,0003256 & 0,0658861 \\
\hline 203 & 0,937821689 & 0,917186603 & 0,9381786 & 0,0003569 & 0,0209920 \\
\hline 204 & 0,814322976 & 0,814105118 & 0,8145964 & 0,0002734 & 0,0004912 \\
\hline 205 & 0,406421949 & 0,326318149 & 0,4064522 & 0,0000302 & 0,0801340 \\
\hline 206 & $-0,143521113$ & $-0,26315563$ & $-0,1436775$ & $-0,0001563$ & 0,1194782 \\
\hline 207 & $-0,643240608$ & $-0,70210221$ & $-0,6436164$ & $-0,0003758$ & 0,0584858 \\
\hline 208 & $-0,918316072$ & $-0,90743172$ & $-0,9187216$ & $-0,0004056$ & $-0,0112899$ \\
\hline 209 & $-0,872602371$ & $-0,81484461$ & $-0,8728910$ & $-0,0002886$ & $-0,0580463$ \\
\hline 210 & $-0,521984957$ & $-0,48570343$ & $-0,5221344$ & $-0,0001494$ & $-0,0364309$ \\
\hline
\end{tabular}

Tabela 7.10 - Análise de entrada randômica com envoltória de $40 \%$ do valor da amplitude do sinal de entrada 


\subsection{QUINTO EXEMPLO: ANÁLISE DE ENTRADA RANDÔMICA PURA}

Para melhor verificar o comportamento do algoritmo de filtragem, será analisada uma entrada contendo somente valores randômicos. Para melhor visualização, a análise se dá em forma de gráfico. Os valores randômicos utilizados variam de $-0,4$ a $+0,4$.

Inicialmente o filtro utilizado é o hermitiano de dois pólos, com corte $\omega_{\mathrm{c}}=50 \mathrm{rad} / \mathrm{s}$ e $\Delta \mathbf{t}=\mathbf{0 , 0 2 \mathrm { s }}$. A figura 7.2 demonstra um arranjo aleatório com sua respectiva saída filtrada. Nota-se que os valores de resposta são praticamente zero.

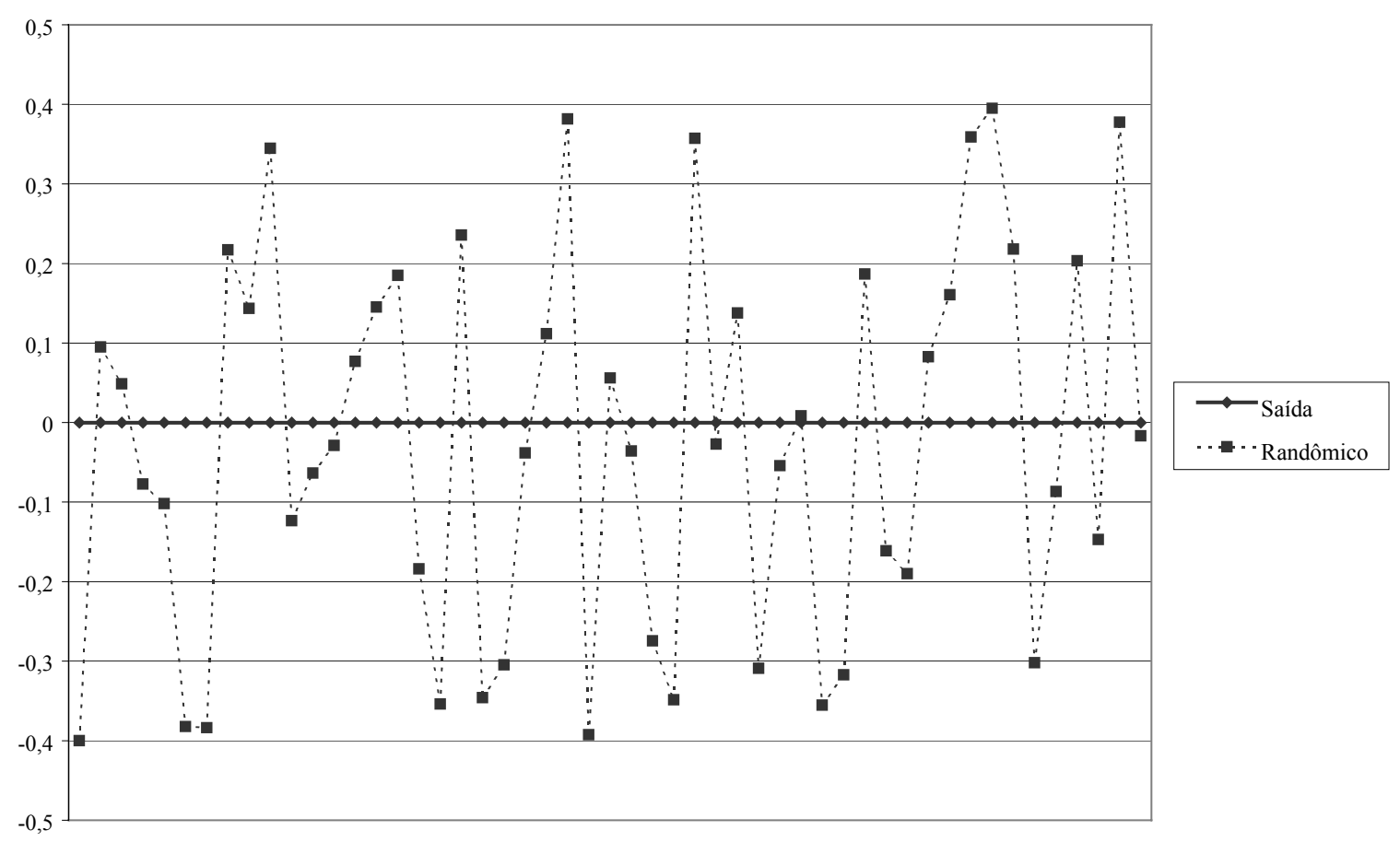

Fig. 7.2 - Filtragem de sinal puramente randômico com filtro de dois pólos 
A figura 7.3 utiliza o mesmo exemplo anterior, porém com um filtro de dez pólos:

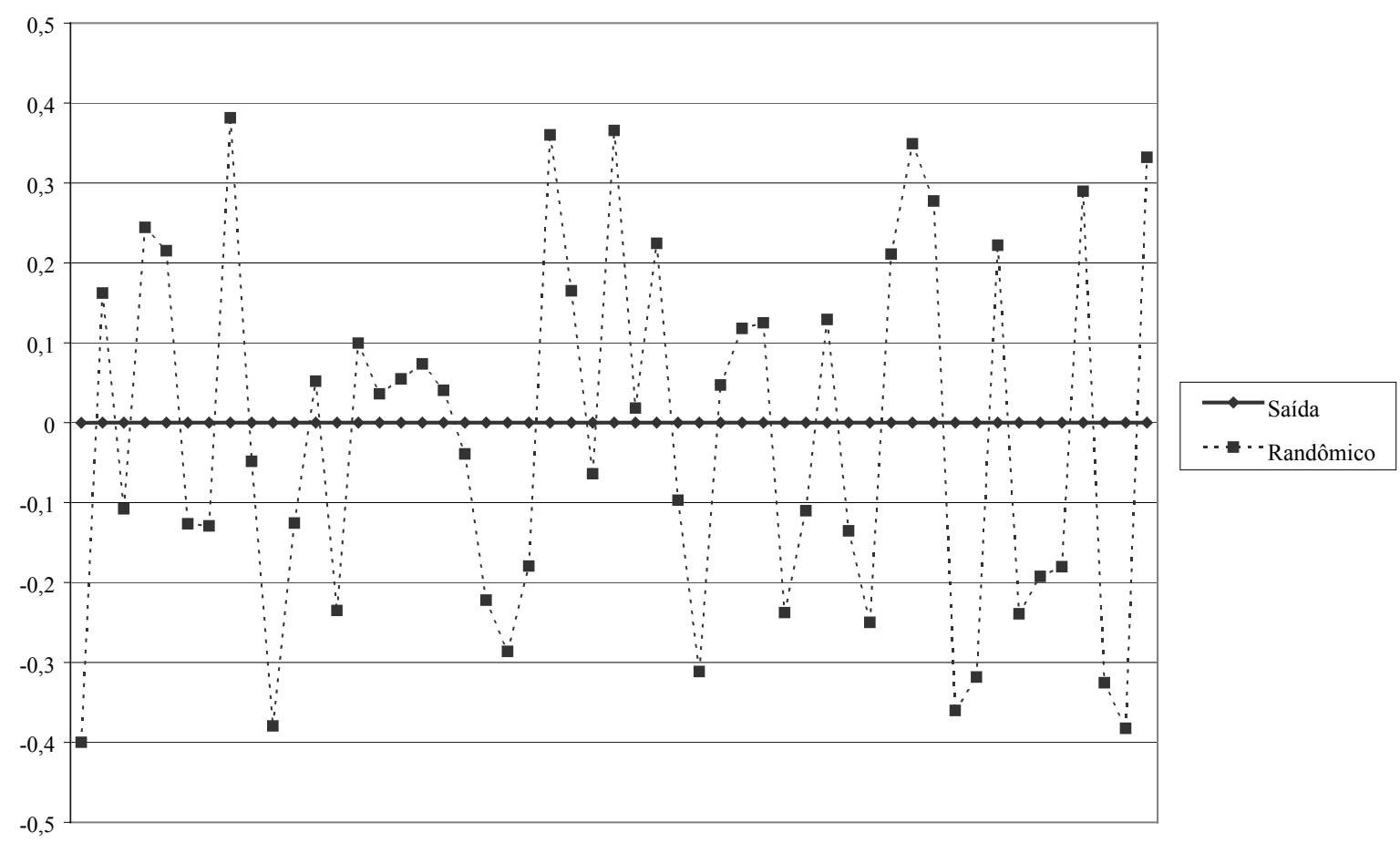

Fig. 7.3 - Filtragem de sinal puramente randômico com filtro de dez pólos

A figura 7.4 utiliza o mesmo exemplo com um filtro de quinze pólos:

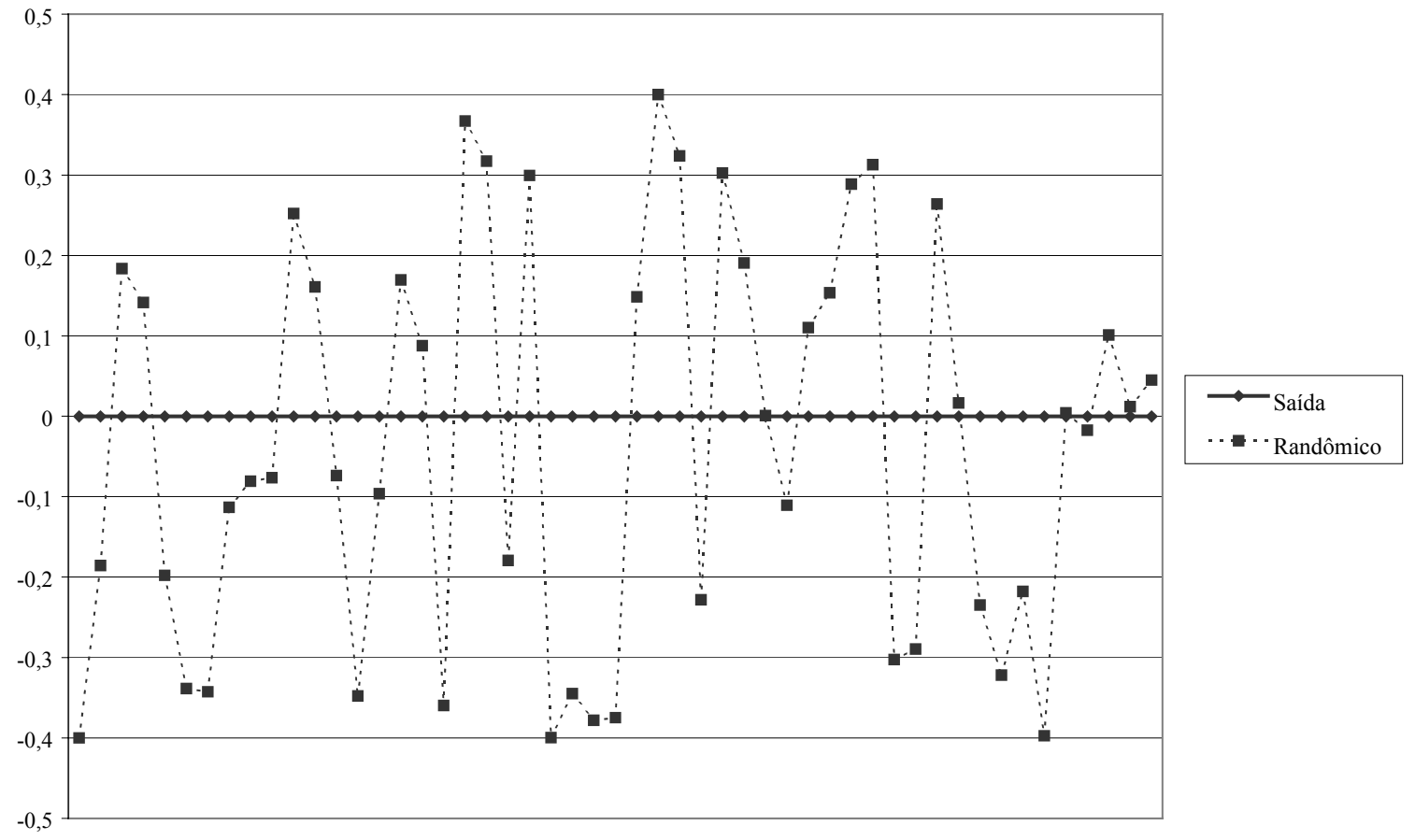

Fig. 7.4 - Filtragem de sinal puramente randômico com filtro de quinze pólos

Percebe-se que a combinação aleatória não influi no resultado. 


\section{Capítulo VIII}

\section{ALGORITMO DE FILTRAGEM PROVENIENTE DA TRANSFORMADA Z}

\subsection{INTRODUÇÃO}

Diante da existência dos métodos de resolução numérica do processo de filtragem já elaborados pelos engenheiros elétricos, torna-se necessária a comparação dos algoritmos de filtragem obtidos neste estudo com o mais comum destes métodos.

No campo da engenharia elétrica, mais precisamente na área de processamento de sinais, a literatura $[1 ; 4]$ indica um tratamento numérico do processo de filtragem de sinal através da discretização da transformada de Laplace, doravante denominada transformada $Z$.

Comenta-se sobre as principais propriedades destas transformadas, seguido de aplicação no processo de filtragem de sinal, com conseqüente exemplificação numérica para filtro de dois pólos juntamente com a comparação destes resultados com os obtidos pelos algoritmos propostos por este estudo. 


\subsection{TRANSFORMADA DE LAPLACE}

O tratamento dado pelos engenheiros elétricos na resolução de uma equação diferencial é a transformada de Laplace, cuja obtenção é similar à transformada de Fourier. Para uma função $f(t)$ (usualmente designada pela forma minúscula) a transformada de Laplace $F(s)$ (usualmente designada pela forma maiúscula) de f(t) é dada como:

$$
F(s)=\int_{0}^{\infty} f(t) e^{-s t} d t
$$

onde s é um número complexo resumido na nomenclatura:

$$
\mathrm{s}=\sigma+\mathrm{i} \omega
$$

A transformada de Fourier provém do caso particular onde $\sigma=0$, ou seja, trata-se da análise apenas no eixo imaginário do plano de Gauss.

$O$ princípio da linearidade e a propriedade das derivadas também estão presentes. A transformada de Laplace da derivada de ordem $\mathrm{N}$ de uma função $f(t)$ com condições iniciais nulas é dada como:

$$
\int_{0}^{\infty} \frac{d^{N} f(t)}{d t^{N}} e^{-s t} d t=s^{N} F(s)
$$

cuja demonstração é análoga ao caso da transformada de Fourier.

Porém, uma particularidade da transformada de Laplace é o fato de sua inversa ser composta por uma integral curvilínea em s, com diferencial ds.

\subsection{TRANSFORMADA Z}

A forma discreta da integração da transformada de Laplace é um somatório denominado transformada Z. No caso da transformada de Fourier, a discretização se dá através da DFT (Discrete Fourier Transform). Na transformada $Z$, a função $f(t)$ tem discretização com um passo de tempo $\Delta t$ e simbolizada com colchetes na forma f[n], onde: 


$$
f[n]=f(n . \Delta t)
$$

A integral (8.1) torna-se assim um somatório expresso por:

$$
F[z]=\sum_{n=0}^{\infty} f[n] z^{-n}
$$

onde $z$ é um número complexo e $F[z]$ representa a transformada $Z$ de f[n]. Vale assinalar que este somatório tem restrições em z para que haja convergência.

Admitindo-se que $f(t)$ é uma função causal, ou seja, não se tenham valores de $f(t)$ para $t<0$, demonstra-se a propriedade principal da transformada $Z$ no processo de filtragem. Seja então aplicada a transformada $Z$ em f[n-m], onde $n>m$, ou seja:

$$
\sum_{n=0}^{\infty} f[n-m] z^{-n}
$$

e efetuando uma mudança de variáveis na forma:

$$
\mathrm{k}=\mathrm{n}-\mathrm{m}
$$

a expressão (8.6) se torna:

$$
\sum_{k+m=0}^{\infty} f[k] z^{-k-m}=z^{-m} \sum_{k=-m}^{\infty} f[k] z^{-k}
$$

onde o somatório no segundo membro da igualdade (8.8), devido ao fato de $f[k]=0$ para $k<0$, iguala-se à transformada $Z$ da função $f(t)$ discretizada, permitindo reescrever (8.8) como sendo:

$$
\sum_{n=0}^{\infty} f[n-m] z^{-n}=z^{-m} F[z]
$$

implicando que a inversa $Z$ de $z^{-m} F[z]$ é $f[n-m]$. 


\subsection{TRANSFORMAÇÃO BILINEAR}

A transformação bilinear relaciona a variável $z$ e a variável $s$ de Laplace. Tendo-se em vista não sobrecarregar o assunto, será efetuada a aplicação para uma equação diferencial de primeira ordem, adiantando que a relação é válida para uma equação diferencial de maior ordem. Uma equação diferencial de primeira ordem pode ser generalizada como sendo:

$$
\mathrm{a}_{1} \dot{\mathrm{y}}(\mathrm{t})+\mathrm{a}_{0} \mathrm{y}(\mathrm{t})=\mathrm{b}_{0} \mathrm{x}(\mathrm{t})
$$

Seja $y[n]$ a forma discreta de $y(t)$ e disposto na forma:

$$
y[n]=y[n-1]+\int_{(n-1) \Delta t}^{n \Delta t} y(t) d t
$$

e utilizando um integrador trapezoidal:

$$
\int_{(n-1) \Delta t}^{n \Delta t} y(t) d t=\frac{\Delta t}{2}\left(\left.\dot{y}(t)\right|_{t=n \Delta t}+\left.\dot{y}(t-\Delta t)\right|_{t=n \Delta t}\right)
$$

com a substituição de (8.10) em (8.12), tem-se:

$$
\int_{(n-1) \Delta t}^{n \Delta t} y(t) d t=\frac{\Delta t}{2 a_{1}}\left(\left.b_{0} x(t)\right|_{t=n \Delta t}-\left.a_{0} y(t)\right|_{t=n \Delta t}+\left.b_{0} x(t-\Delta t)\right|_{t=n \Delta t}-\left.a_{0} y(t-\Delta t)\right|_{t=n \Delta t}\right)
$$

onde a substituição de (8.13), na forma já discretizada, em (8.11) conduz a:

$$
a_{1} y[n]=a_{1} y[n-1]+\frac{\Delta t}{2}\left(b_{0} x[n]-a_{0} y[n]+b_{0} x[n-1]-a_{0} y[n-1]\right)
$$

A transformação bilinear conduz a uma relação entre a variável da transformada Z e a variável da transformada de Laplace para uma equação diferencial. Por se tratar de uma relação entre entrada $(x(t))$ e saída $(y(t))$, denota-se $H(s)$ a relação $Y(s) / X(s)$ e $H[z]$ a relação $Y[z] / X[z]$. Assim sendo, tomando-se a transformada inversa $Z$ de (8.14):

$$
\mathrm{a}_{1} \mathrm{Y}[\mathrm{z}]-\mathrm{a}_{1} \mathrm{z}^{-1} \mathrm{Y}[\mathrm{z}]+\mathrm{a}_{0} \frac{\Delta \mathrm{t}}{2}\left(\mathrm{Y}[\mathrm{z}]+\mathrm{z}^{-1} \mathrm{Y}[\mathrm{z}]\right)=\mathrm{b}_{0} \frac{\Delta \mathrm{t}}{2}\left(\mathrm{X}[\mathrm{z}]+\mathrm{z}^{-1} \mathrm{X}[\mathrm{z}]\right)
$$

e reorganizando os termos, tem-se: 


$$
\frac{\mathrm{Y}[\mathrm{z}]}{\mathrm{X}[\mathrm{z}]}=\mathrm{H}[\mathrm{z}]=\frac{\mathrm{b}_{0}}{\mathrm{a}_{1} \frac{2}{\Delta \mathrm{t}} \frac{1-\mathrm{z}^{-1}}{1+\mathrm{z}^{-1}}+\mathrm{a}_{0}}
$$

A relação $H(s)$ é facilmente obtida conseguindo-se a transformada de Laplace da equação (8.10), que conduz a:

$$
\frac{Y(s)}{X(s)}=H(s)=\frac{b_{0}}{a_{1} s+a_{0}}
$$

e a partir da igualdade entre (8.16) e (8.17) tem-se:

$$
\mathrm{s} \rightarrow \frac{2}{\Delta \mathrm{t}} \frac{1-\mathrm{z}^{-1}}{1+\mathrm{z}^{-1}}
$$

representando assim a transformação bilinear. Para a relação $H(s)$ de uma equação de filtragem de maior número de pólos, a expressão (8.18) também é válida.

\subsection{POLINÔMIO DE BUTTERWORTH}

A equação de filtragem pode ser obtida através da transformada de Laplace de forma similar à transformada de Fourier. O processo se baseia em modelar $|\mathrm{H}(\mathrm{s})|$ para que se consiga:

$$
|\mathrm{H}(\mathrm{s})|=\frac{1}{\sqrt{1+\frac{\omega^{2 \mathrm{~N}}}{\omega_{\mathrm{c}}}}}
$$

com conseqüente atribuição na forma:

$$
s \rightarrow \frac{i \omega}{\omega_{c}}
$$

onde $\omega_{c}$ é a freqüência de corte.

A variável complexa s de Laplace, de acordo com (8.2), possui uma parte real $\sigma$ responsável pela parte transitória da solução da equação diferencial e uma parte imaginária $\omega$ correspondente à solução permanente do sistema. Demonstrando de maneira simplificada, obtém-se as raízes $\omega_{\kappa}$ de: 


$$
1+\left(\frac{\omega}{\omega_{c}}\right)^{2 \mathrm{~N}}=0
$$

e multiplica-se pela unidade complexa para se encontrar as raízes $s_{k}$, escolhendo os pólos do lado esquerdo do plano de Gauss, por possuírem a parte real negativa, para compor o polinômio de Butterworth $\mathrm{B}(\mathrm{s})$, análogo ao polinômio $P(\omega)$ proveniente da transformada de Fourier. Com isso, $H(s)$ é do tipo:

$$
H(s)=\frac{1}{B(s)}
$$

Para o caso de dois pólos, B(s) é:

$$
B(s)=s^{2}+\sqrt{2} s+1
$$

onde pode-se notar que fazendo $s=i \omega / \omega_{c}$, obtém-se $P(\omega)$ para dois pólos.

\subsection{DESENVOLVIMENTO DE FILTRO DIGITAL DE DOIS PÓLOS}

Uma vez demonstrado brevemente o embasamento teórico da equação de filtragem segundo a transformada de Laplace juntamente com sua forma discretizada, parte-se para a obtenção do algoritmo de filtragem.

A função $H(s)$ para dois pólos é:

$$
H(s)=\frac{1}{s^{2}+\sqrt{2} s+1}
$$

que para a mudança de escala segundo (8.20), $\mathrm{H}(\mathrm{s})$ adquire a forma:

$$
H(s)=\frac{1}{\frac{1}{\omega_{c}^{2}} s^{2}+\frac{1}{\omega_{c}} \sqrt{2} s+1}
$$

Aplicando agora a transformação bilinear em (8.25), tem-se:

$$
\left.\mathrm{H}(\mathrm{s})\right|_{\mathrm{s} \rightarrow \frac{2}{\Delta \mathrm{t}} \frac{1-\mathrm{z}^{-1}}{1+\mathrm{z}^{-1}}}=\mathrm{H}[\mathrm{z}]=\frac{1}{\frac{1}{\omega_{\mathrm{c}}^{2}}\left(\frac{2}{\Delta \mathrm{t}} \frac{1-\mathrm{z}^{-1}}{1+\mathrm{z}^{-1}}\right)^{2}+\frac{1}{\omega_{\mathrm{c}}} \sqrt{2}\left(\frac{2}{\Delta \mathrm{t}} \frac{1-\mathrm{z}^{-1}}{1+\mathrm{z}^{-1}}\right)+1}
$$


e simplificando:

$$
H[z]=\frac{\theta^{2}+2 \theta^{2} z^{-1}+\theta^{2} z^{-2}}{\left(4+2 \theta \sqrt{2}+\theta^{2}\right)+\left(2 \theta^{2}-8\right) z^{-1}+\left(4-2 \theta \sqrt{2}+\theta^{2}\right) z^{-2}}=\frac{Y[z]}{X[z]}
$$

onde novamente $\theta$ vale $\omega_{c} \Delta$ t. Vale observar neste ponto do desenvolvimento que, de acordo com a primeira parcela do denominador do último membro de (8.26), a maior potência negativa de z é igual ao número de pólos do filtro. Rearranjando (8.27) na forma:

$$
\left\lfloor\left(4+2 \theta \sqrt{2}+\theta^{2}\right)+\left(2 \theta^{2}-8\right) \mathrm{z}^{-1}+\left(4-2 \theta \sqrt{2}+\theta^{2}\right) \mathrm{z}^{-2}\right] \mathrm{Y}[\mathrm{z}]=\left[\theta^{2}+2 \theta^{2} \mathrm{z}^{-1}+\theta^{2} \mathrm{z}^{-2}\right] \mathrm{X}[\mathrm{z}]
$$

e tomando-se a inversa da transformada $Z$ em ambos os membros:

$$
\begin{aligned}
& \left(4+2 \theta \sqrt{2}+\theta^{2}\right) y[n]+\left(2 \theta^{2}-8\right) y[n-1]+\left(4-2 \theta \sqrt{2}+\theta^{2}\right) y[n-2]= \\
& \theta^{2} x[n]+2 \theta^{2} x[n-1]+\theta^{2} x[n-2]
\end{aligned}
$$

o que representa um algoritmo do tipo:

$$
\begin{aligned}
& y[n]=\frac{\theta^{2}}{\left(4+2 \theta \sqrt{2}+\theta^{2}\right)} x[n]+\frac{2 \theta^{2}}{\left(4+2 \theta \sqrt{2}+\theta^{2}\right)} x[n-1]+ \\
& \frac{\theta^{2}}{\left(4+2 \theta \sqrt{2}+\theta^{2}\right)} x[n-2]-\frac{\left(2 \theta^{2}-8\right)}{\left(4+2 \theta \sqrt{2}+\theta^{2}\right)} y[n-1]-\frac{\left(4-2 \theta \sqrt{2}+\theta^{2}\right)}{\left(4+2 \theta \sqrt{2}+\theta^{2}\right)} y[n-2]
\end{aligned}
$$

Para o caso de um número $\mathrm{N}$ de pólos, o algoritmo utiliza $\mathrm{N}+1$ valores de entrada e $\mathrm{N}$ valores de saída. Nota-se, portanto, que (8.30) representa um algoritmo expresso por uma relação algébrica simples e de múltiplo passo, diferentemente do algoritmo proposto por este estudo que utiliza um algoritmo expresso por uma relação matricial de único passo.

\subsection{COMPARAÇÃO DOS RESULTADOS}

Aplicando na expressão (8.30) uma freqüência de corte $\omega_{c}=50 \mathrm{rad} / \mathrm{s}$ e uma discretização com $\Delta \mathbf{t}=\mathbf{0 , 0 3}$ do sinal de entrada do tipo:

$$
x(t)=\operatorname{sen}(30 t)
$$


e comparando o resultado com o obtido através dos algoritmos propostos no trabalho, tem-se tabela 8.1:

\begin{tabular}{|c|c|c|c|c|c|c|c|}
\hline \multirow[b]{2}{*}{$\mathrm{i}$} & \multicolumn{3}{|c|}{ Resultados dos algoritmos } & \multirow[b]{2}{*}{$\begin{array}{l}\text { Resultado } \\
\text { teórico }\end{array}$} & \multicolumn{3}{|c|}{ Erro local dos algoritmos } \\
\hline & $\begin{array}{c}\text { Transformada } \\
Z\end{array}$ & Trapezoidal & Hermitiano & & $\begin{array}{c}\text { Transformada } \\
Z\end{array}$ & Trapezoidal & Hermitiano \\
\hline 200 & 0,065099 & 0,065099 & $-0,001770$ & $-0,0043621$ & $-0,069461$ & $-0,069461$ & $-0,002592$ \\
\hline 201 & $-0,681276$ & $-0,681276$ & $-0,734281$ & $-0,7397261$ & $-0,058450$ & $-0,058450$ & $-0,005445$ \\
\hline 202 & $-0,912075$ & $-0,912075$ & $-0,911133$ & $-0,9152800$ & $-0,003205$ & $-0,003205$ & $-0,004147$ \\
\hline 203 & $-0,452634$ & $-0,452634$ & $-0,398488$ & $-0,3981683$ & 0,054466 & 0,054466 & 0,000320 \\
\hline 204 & 0,349352 & 0,349352 & 0,415694 & 0,4202692 & 0,070918 & 0,070918 & 0,004575 \\
\hline 205 & 0,886955 & 0,886955 & 0,915258 & 0,9206554 & 0,033700 & 0,033700 & 0,005398 \\
\hline 206 & 0,753329 & 0,753329 & 0,722142 & 0,7243079 & $-0,029021$ & $-0,029021$ & 0,002166 \\
\hline 207 & 0,049598 & 0,049598 & $-0,017507$ & $-0,0201813$ & $-0,069779$ & $-0,069779$ & $-0,002675$ \\
\hline 208 & $-0,691667$ & $-0,691667$ & $-0,743937$ & $-0,7493978$ & $-0,057730$ & $-0,057730$ & $-0,005461$ \\
\hline 209 & $-0,909493$ & $-0,909493$ & $-0,907401$ & $-0,9114849$ & $-0,001992$ & $-0,001992$ & $-0,004084$ \\
\hline 210 & $-0,439032$ & $-0,439032$ & $-0,384192$ & $-0,3837784$ & 0,055254 & 0,055254 & 0,000413 \\
\hline
\end{tabular}

Tabela 8.1 - Comparação dos algoritmos para $\Delta \mathrm{t}=0.03 \mathrm{~s}$

Nota-se claramente que, devido ao fato da quadratura numérica da transformada $Z$ ser a mesma que a do algoritmo trapezoidal deste estudo, os resultados destes dois algoritmos são iguais. 


\section{Capítulo IX}

\section{OBSERVAÇÕES FINAIS E CONCLUSÕES}

Em primeiro lugar, cabe assinalar que foram duas as principais contribuições do presente trabalho para a análise dinâmica de estruturas. A primeira delas, a mais importante, foi uma apropriada leitura processo de filtragem de freqüência, traduzindo-se a redação de tal processo, por assim se dizer, para uma linguagem mais facilmente inteligível para a engenharia de estruturas. A segunda contribuição, de cunho mais inédito, foi o desenvolvimento de algoritmos incondicionalmente estáveis e com alta precisão para a filtragem digital de freqüências segundo o clássico modelo de Butterworth.

O emprego de operadores hermitianos de ordem elevada [6] na elaboração de algoritmos de integração das equações diferenciais correspondentes aos filtros de Butterworth se mostrou bastante sugestivo. Os operadores hermitianos em questão foram elaborados procurando-se, como mostrado, aumentar a ordem de precisão do integrador, sem deixar de se utilizar a importante característica de se tratar e um algoritmo matricial de passo único, no qual, para a consideração das condições iniciais, diferentemente do que ocorre com os algoritmos de múltiplos passos, bastante usuais na engenharia elétrica, não é necessário ter-se em conta a elaboração 
de algoritmos especiais. É interessante também ser observado que a forma mais simples deste operador consiste no clássico integrador trapezoidal, muito empregado na engenharia de estruturas. Todavia, tal operador é empregado na dinâmica das estruturas gerando-se algoritmos de integração de passo único, e não como preconizado pela via da chamada transformada $Z$, conduzindo-se a um algoritmo de integração de passo duplo. Neste trabalho, foi elaborado um algoritmo com erro local de integração de quinta ordem, e indicados os procedimentos para a obtenção de algoritmos com ordem maior de precisão. Os resultados obtidos são, naturalmente, de maior precisão, mas é necessário ressaltar que, conforme a combinação de operadores hermitianos envolve mais valores de derivadas de maior ordem, torna-se necessário contar também com uma maior discretização do sinal de entrada, que deve ter ordem de erro compatível com a do integrador sendo considerado.

Verificou-se em alguns exemplos de aplicação que o algoritmo hermitiano mostrou-se capaz de filtrar um sinal, mesmo quando considerada uma componente aleatória no sinal de entrada (um eventual erro aleatório de leitura do aparelho de mensuração, por exemplo). Pode-se justificar tal comportamento pelo fato de que uma eventual regularidade na freqüência do sinal aleatório não dispor de duração suficiente para mobilizar valores na resposta em regime permanente (tempo de resposta do filtro), como sucede com a componente harmônica presente.

Vale ressaltar que a redução da equação diferencial de filtragem a um sistema de equações diferenciais de primeira ordem considerada neste trabalho pode ser aplicada não só para o caso do filtro de Butterworth, mas também para o do filtro de Chebyschev, do filtro elíptico e outros, dado que também possuem suas respectivas equações diferenciais no domínio do tempo, resultando-se, naturalmente, em diferentes algoritmos de filtragem de freqüência. Aliás, tal redução apresentada pode ser aplicada para formular a integração de qualquer equação diferencial. 
Finalizando-se, acredita-se ter sido dada uma contribuição para um melhor entendimento do tema por parte da engenharia de estruturas, e também contribuído positivamente propondo-se uma formulação mais precisa de integração das equações diferenciais de filtragem. 


\section{Referências bibliográficas}

1- LUDEMAN, Lonnie C. - Fundamentals of Digital Signal Processing, New Mexico State University, 1987

2- WARBURTON, Geoffrey B. - The Dynamical Behavior of Structures, Oxford, Pergamon Press, 1976

3- SPIEGEL, Murray R. - Análise de Fourier (Coleção Schaum), Editora McGraw-Hill do Brasil, 1976

4- $\quad$ CARLSON, Gordon E. - Signal and Linear System Analysis, University of Missouri, 1998

5- $\quad$ ASCHER, Uri M., Robert M. M., Robert D. Russel - Numerical Solution of Boundary Value Problems for Ordinary Differential Equation, Philadelphia: Society for Industrial and Applied Mathematics, 1995

6- LAIER, José Elias - High Order Hermitian Algorithm of Integration in Time, $15^{\text {th }}$ ASCE Engineering Mechanics Conference, Columbia University, New York, NY, 2002 